Susumu Umino, Yuki Kusano, Atsushi Yamaji, Takahiro Fudai, Akihiro Tamura, and Shoji Arai, 2019, The conversion tectonics from spreading to subduction: Paleostress analysis of dike swarms during the subduction initiation in the Oman Ophiolite: GSA Bulletin, https:// doi.org/10.1130/B35202.1.

\title{
Analytical procedures and uncertainties
}

Whole rock major element analyses were done by an XRF method using the ZSX primus II of the College of Science and Engineering, Kanazawa University (Table S2). Mixtures of sample :

lithium tetraborate at a ratio of 1:10 were fused to form glass beads, which were utilized for XRF analyses. Analytical procedures are described in Kusano et al. (2014). Analytical errors were evaluated on repeated analyses of international standard samples, BHVO-2, BCR-2 and BIR-1. Representative relative errors of analyses (relative deviations from recommended values) are better than $1.1 \%$ for $\mathrm{SiO}_{2}, \mathrm{MnO}, \mathrm{MgO}, \mathrm{CaO}, \mathrm{K}_{2} \mathrm{O},<2.7 \%$ for $\mathrm{TiO}_{2}, \mathrm{Al}_{2} \mathrm{O}_{3}, \mathrm{Fe}_{2} \mathrm{O}_{3}, \mathrm{Na}_{2} \mathrm{O},<3.7 \%$ for $\mathrm{P}_{2} \mathrm{O}_{5}$.

Trace element compositions (REEs, V, Cr, Co, Ni, Li, B, Sc, Rb, Sr, Y, Zr, Nb, Cs, Ba, Hf, Ta and Th) of whole-rock fused glass were analyzed by laser ablation (193 nm ArF excimer: MicroLas GeoLas Q-plus) inductively coupled plasma mass spectrometry (Agilent 7500s) (LA-ICP-MS) at Kanazawa University. Details of the analytical method and data quality for the LA-ICP-MS system at Kanazawa University are described in Tamura et al. (2015). Whole-rock powder was fused on an Ir-strip heater connected to copper electrodes after Nicholls (1974) and Stoll et al. (2008). Before fusing experiments, Ir strips were cleaned with $5 \% \mathrm{HCl}$ and ultrapure water. Approximately $20 \mathrm{mg}$ of rock powder was put on an Ir strip 30-50 $\mathrm{mm}$ in length and $5 \mathrm{~mm}$ in width with a thickness of 
quenched by cutting off the power and blowing air. Each analysis was performed by ablating spots of $100 \mu \mathrm{m}$ in diameter at $5 \mathrm{~Hz}$ with energy density of $8 \mathrm{~J} / \mathrm{cm}^{2}$ per pulse. Signal integration times were 50 seconds for a gas background interval and 50 seconds for an ablation interval. BCR-2G (USGS glass reference material) was used as a primary calibration standard and its element concentration values are selected from the GeoReM database (see Jochum \& Nohl, 2008). Data reduction was facilitated using ${ }^{42} \mathrm{Ca}$ as internal standards for glass, based on $\mathrm{CaO}$ contents obtained by XRF analysis, and followed a protocol essentially identical to that outlined by Longerich et al (1996). Fused glasses of powdered geochemical reference materials (BHVO-2, JB-2 and JA-2) were prepared and analyzed with our samples for the quality control of the analytical method. Relative deviations from reference values are better than $10 \%$ for most of the elements (Table S2).

\section{A GArcmB}

GArcmB (Yamaji et al., 2010; Yamaji and Sato, 2011; Yamaji, 2016a, b) fits a mixed Bingham distribution to dike orientations and estimates sets of stress axis orientations by the fuzzy clustering of the dike poles as described in the text. The optimal number of clusters, $K^{\mathrm{opt}}$, is determined to minimize the Bayesian information criterion, BIC (Fig. S1). Once the stress orientation and stress ratio are determined for each cluster, pairs of normal and shear stresses of individual dikes are calculated and shown on Mohr diagrams (Fig. S2). Each hemisphere represents normal and shear stresses acting on a plane parallel to $\sigma_{1}, \sigma_{2}$, and $\sigma_{3}$. Each data point represents a pair of normal and shear stresses acting on a dike plane oblique to the principle 
stress axes, which plot within the area bounded by three Mohr hemispheres. The data point with the highest normal stress value of each cluster indicate the minimum estimate of the maximum magma pressure (Fig. S2). The probability for a dike belonging to each cluster is represented by the gradational color scales.

\section{References}

Jochum, K. P., and Nohl, U., 2008, Reference materials in geochemistry and environmental research and the GeoReM database: Chemical Geology, v. 253, p. 50-53.

Kusano, Y., Umino, S., Kobayashi, J., Mizukami, T., Okuno, M., and Arai, S., 2014, Quantitative analysis of major elements in igneous rocks with X-ray fluorescence spectrometer "ZSX primus II" using a 1:10 dilution glass bead: The science reports of the Kanazawa University, v. 58, p. $31-44$.

Longerich, H. P., Jackson, S. E., Gunther, D. (1996) Laser ablation inductively coupled plasma mass spectrometric transient signal data acquisition and analyte concentration calculation: Journal of Analytical Atomic Spectroscopy, v. 11, p. 899-904.

Nicholls, I. A., 1974, A direct fusion method of preparing silicate rock glasses for energy-dispersive electron microprobe analysis: Chemcal Geology, v. 14, p. 151-157.

Stoll, B., Jochum, K.P., Herwig, K., Amini, M., Flanz, M., Kreuzburg, B., Kuzmin, D., Willbold, M., and Enzweiler, J., 2008, An automated iridium-strip heater for LA-ICP-MS bulk analysis of geological samples: Geostandards and Geoanalytical Research, v. 32, p. 5-26. 
Tamura, A., Akizawa, N., Otsuka, R., Kanayama, K., Python, M., Morishita, T., and Arai, S., 2015, Measurement of whole-rock trace-element composition by flux-free fused glass and LA-ICPMS: evaluation of simple and rapid routine work: Geochemical Journal, v. 49, p. 243-259.

Yamaji, A., 2016a, Genetic algorithm for fitting a mixed Bingham distribution to 3D orientations: a tool for the statistical and paleostress analyses of fracture orientations: Island Arc, v. 25, p. $72-$ 1157, doi: 10.1016/j.jsg.2011.05.006.

Yamaji, A., 2016b, GArcmB Software Package User's Guide: http://www.kueps.kyotou.ac.jp/ web-bs/tsg/software/GArcmB/.

Yamaji, A., Sato, K., and Tonai, S., 2010, Stochastic modeling for the stress inversion of vein orientations: Paleostress analysis of Pliocene epithermal veins in southwestern Kyushu, Japan: Journal of Structural Geology, v. 32, p. 1137-1146.

Yamaji, A., and Sato, K.2011, Clustering of fracture orientations using a mixed Bingham distribution and its application to paleostress analysis from dike or vein orientations. : Journal of Structural Geology, v. 33, p. 1148-83, doi: 10.1111/iar.12135. 
(A)

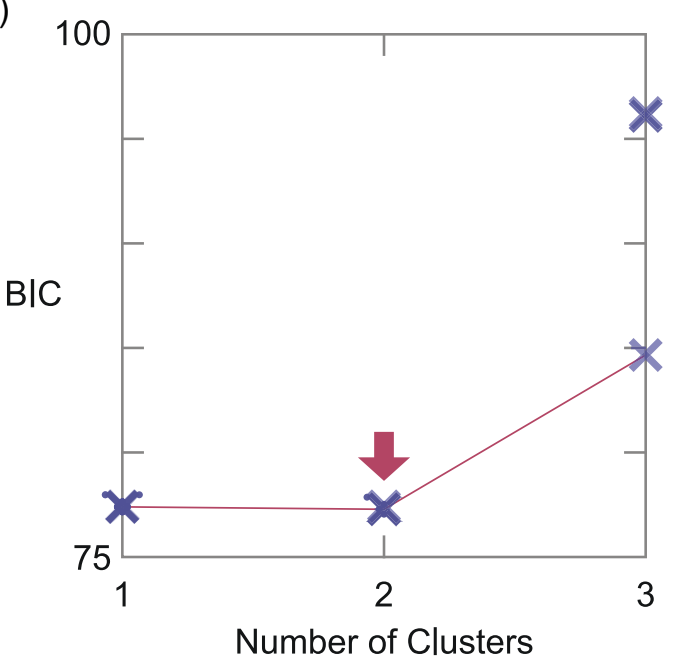

(C)

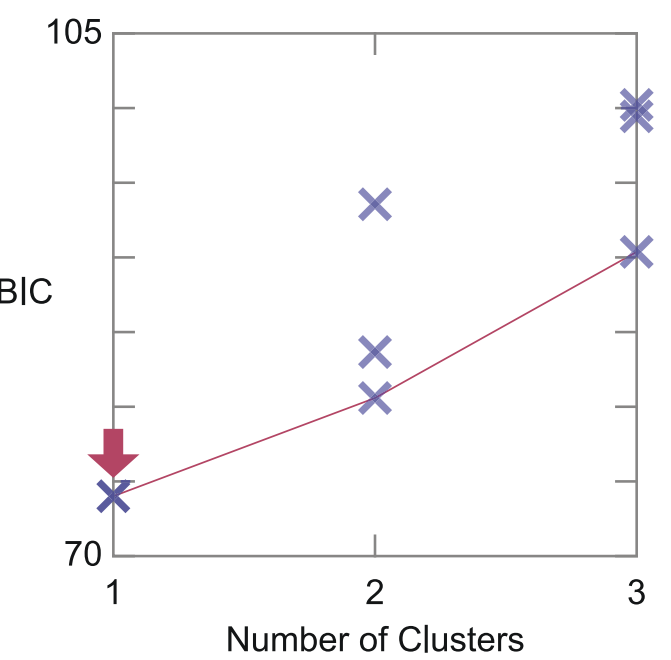

$(\mathrm{E})$

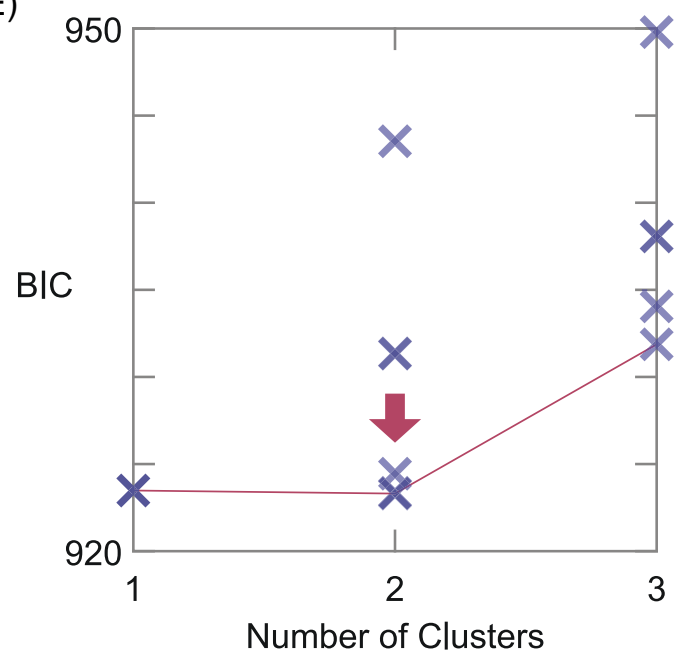

(B)

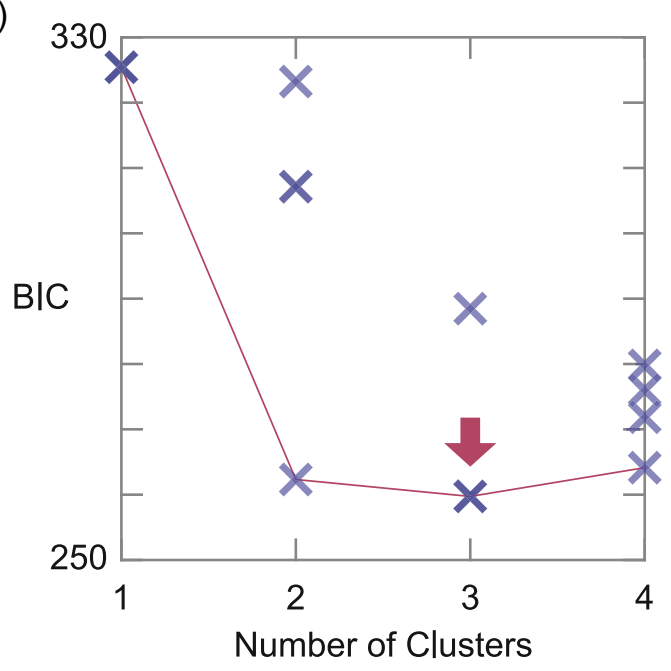

(D)

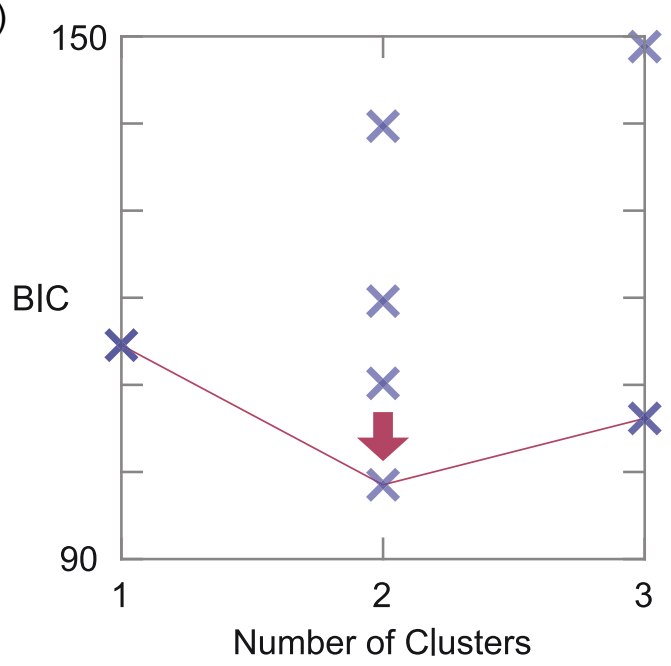

(F)

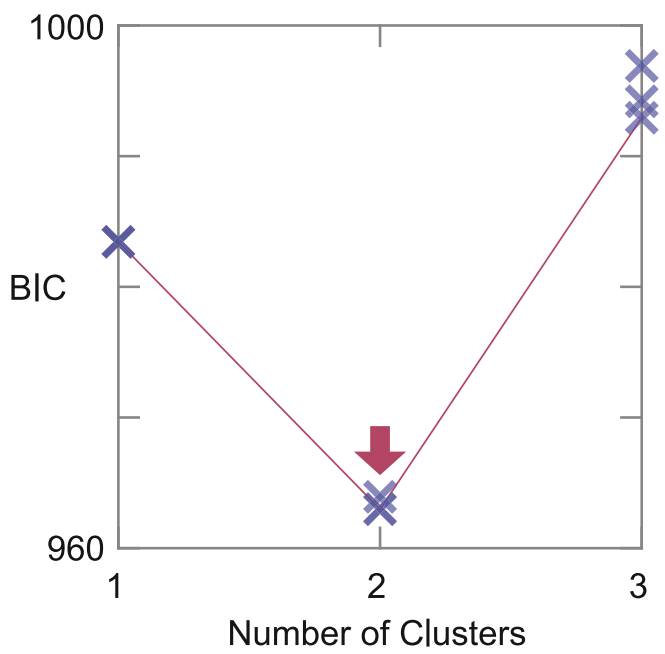

Figure S1. BICs of the analyzed the E-W dike Swarm-1 (A, B), -3 (C, D) , -4 (E) and -5 (F), corresponding to those in Fig. 4, showing the number of clusters that yields the minimum BIC. Please refer to Yamaji and Sato (2011) and Yamaji (2016) for the detail. 


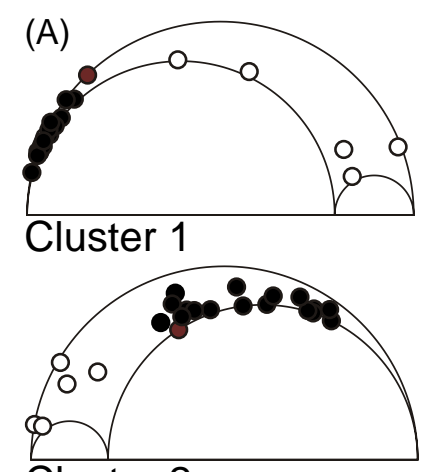

Cluster 2

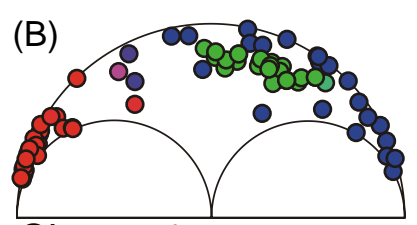

Cluster 1

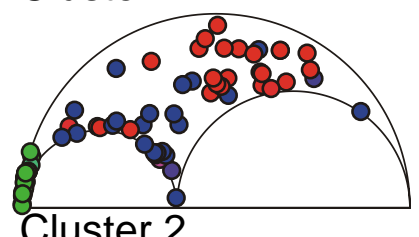

Cluster 2

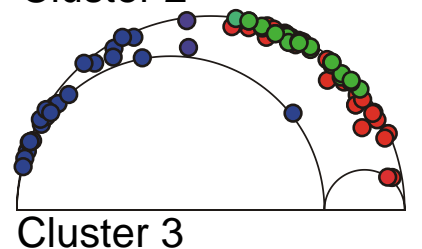

(E)

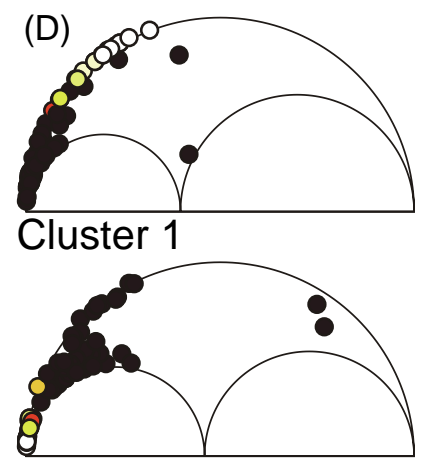

Cluster 2
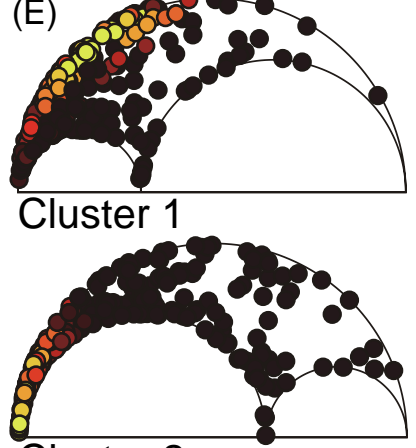

Cluster 2
(G)

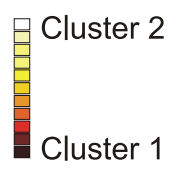

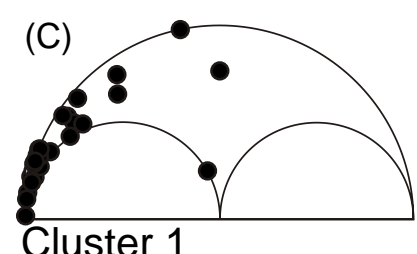

Cluster 1
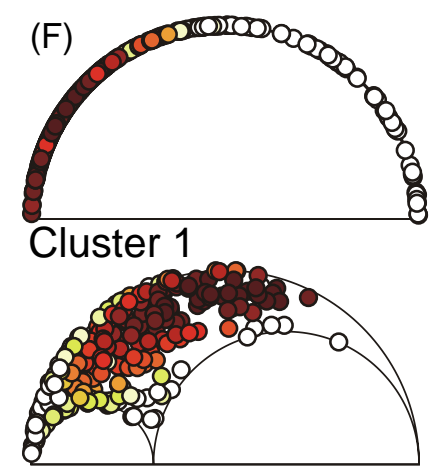

Cluster 2

(H) Cluster 1

Cluster $2 \quad$ Cluster 3

Figure S2. Mohr diagrams of the E-W dike Swarm-1 (A, B), -3 (C, D) , -4 (E) and -5 (F), corresponding to those in Fig. 4. V2 dike Swarm-1 intruded into V1 extrusive rocks (A), sheeted dikes of Swarm-1 (B), dikes in Swarm-3 intruded into the V2 arc stage gabbros (C), sheeted dikes of Swarm-3 (D), all dikes in Swarm-4 (E) and -5 (F). (G) Scale bar for dikes consisting of two clusters (A, D-F) represent probability for the dike belonging to cluster 1 (dark colors) and cluster 2 (light colors). (H) The triangle represents the probability for the dike belonging to cluster 1, 2 and 3, by mixing of red, green and blue, respectively. Please refer to Yamaji and Sato (2011) and Yamaji (2016) for the detail. 
Table S1. Structures of the E-W dike swarms and associated V1 sheeted dikes.

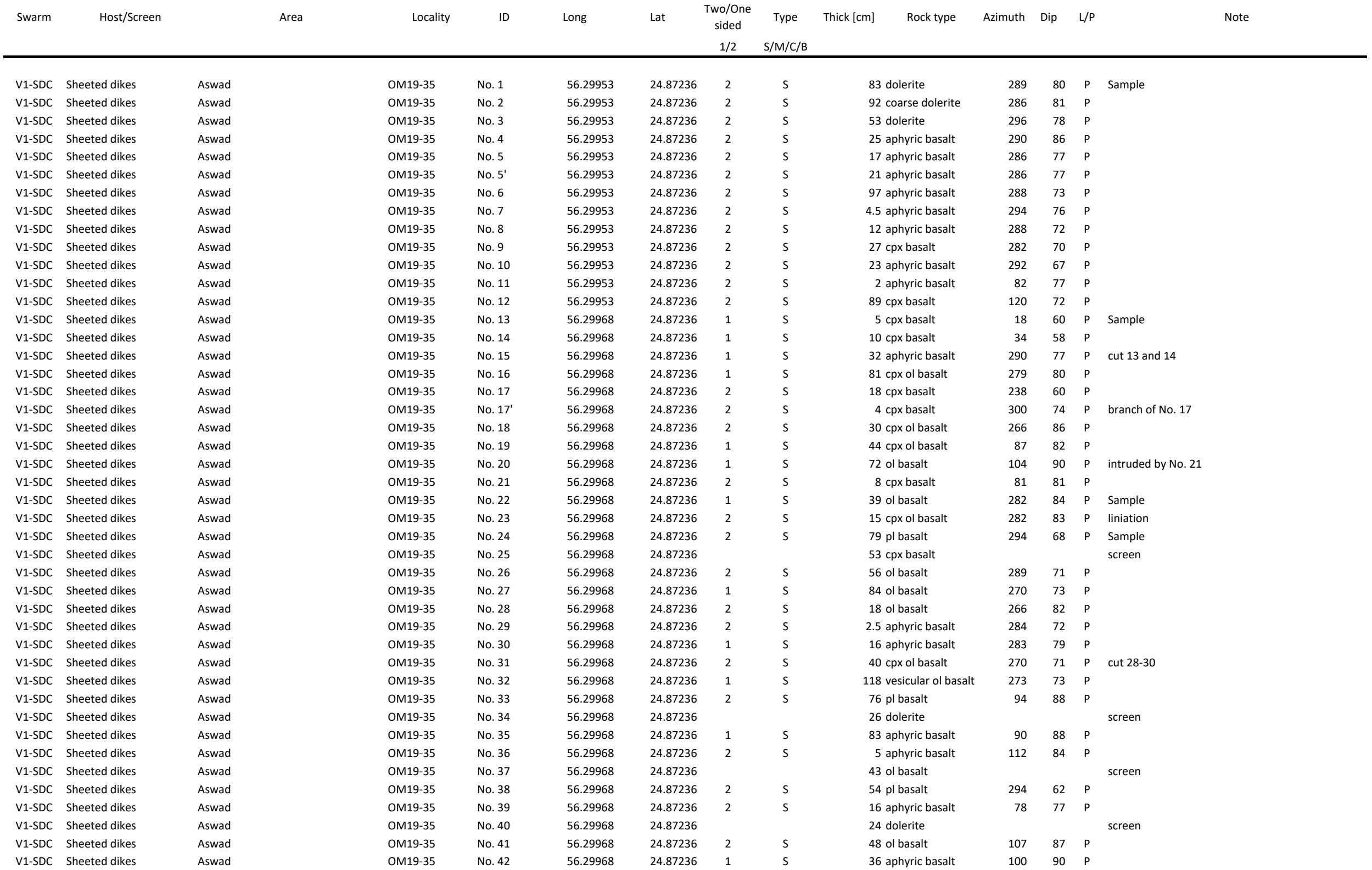




\begin{tabular}{|c|c|c|c|c|c|c|c|c|c|c|c|c|c|}
\hline \multirow[t]{2}{*}{ Swarm } & \multirow[t]{2}{*}{ Host/Screen } & \multirow[t]{2}{*}{ Area } & \multirow[t]{2}{*}{ Locality } & \multirow[t]{2}{*}{ ID } & \multirow[t]{2}{*}{ Long } & \multirow[t]{2}{*}{ Lat } & \multirow{2}{*}{$\begin{array}{c}\text { Two/One } \\
\text { sided } \\
1 / 2\end{array}$} & \multirow{2}{*}{$\begin{array}{c}\text { Type } \\
S / M / C / B\end{array}$} & \multirow[t]{2}{*}{ Thick [cm] } & \multirow[t]{2}{*}{ Azimuth } & \multirow[t]{2}{*}{ Dip } & \multirow[t]{2}{*}{$\mathrm{L} / \mathrm{P}$} & \multirow[t]{2}{*}{ Note } \\
\hline & & & & & & & & & & & & & \\
\hline V1-SDC & Sheeted dikes & Aswad & OM19-35 & No. 43 & 56.29968 & 24.87236 & 1 & $\mathrm{~s}$ & 16 aphyric basalt & 106 & 88 & $\mathrm{P}$ & \\
\hline V1-SDC & Sheeted dikes & Aswad & OM19-35 & No. 44 & 56.29968 & 24.87236 & 1 & s & $114 \mathrm{cpx}$ basalt & 98 & 90 & $\mathrm{P}$ & \\
\hline V1-SDC & Sheeted dikes & Aswad & OM19-35 & No. 45 & 56.29968 & 24.87236 & 2 & s & 16 ol basalt & 298 & 72 & $\mathrm{P}$ & \\
\hline V1-SDC & Sheeted dikes & Aswad & OM19-35 & No. 46 & 56.29968 & 24.87236 & 2 & $\mathrm{~s}$ & 96 aphyric basalt & 294 & 74 & $\mathrm{P}$ & \\
\hline V1-SDC & Sheeted dikes & Aswad & OM19-35 & No. 47 & 56.29968 & 24.87236 & & & $36 \mathrm{cpx}$ basalt & & & & screen \\
\hline V1-SDC & Sheeted dikes & Aswad & OM19-35 & No. 48 & 56.29968 & 24.87236 & 2 & $\mathrm{~s}$ & $100 \mathrm{cpx}$ basalt & 105 & 90 & $\mathrm{P}$ & $\max 5 \mathrm{~mm} \mathrm{cpx}$ \\
\hline V1-SDC & Sheeted dikes & Aswad & OM19-35 & No. 49 & 56.29968 & 24.87236 & 1 & $\mathrm{~s}$ & 22 ol basalt & 305 & 80 & $\mathrm{P}$ & \\
\hline V1-SDC & Sheeted dikes & Aswad & OM19-35 & No. 50 & 56.29968 & 24.87236 & 2 & $\mathrm{~s}$ & 5 aphyric basalt & 282 & 73 & $\mathrm{P}$ & \\
\hline V1-SDC & Sheeted dikes & Aswad & OM19-35 & No. 51 & 56.29968 & 24.87236 & 2 & $\mathrm{~s}$ & $25 \mathrm{cpx}$ basalt & 304 & 65 & $\mathrm{P}$ & \\
\hline V1-SDC & Sheeted dikes & Aswad & OM19-35 & No. 52 & 56.29968 & 24.87236 & 1 & $\mathrm{~s}$ & 34 ol basalt & 300 & 64 & $\mathrm{P}$ & \\
\hline V1-SDC & Sheeted dikes & Aswad & OM19-35 & No. 53 & 56.29968 & 24.87236 & & & 15 dolerite & & & & screen \\
\hline V1-SDC & Sheeted dikes & Aswad & OM19-35 & No. 54 & 56.29968 & 24.87236 & 2 & $\mathrm{~s}$ & 135 aphyric basalt & 288 & 79 & $\mathrm{P}$ & \\
\hline V1-SDC & Sheeted dikes & Aswad & OM19-35 & No. 55 & 56.29968 & 24.87236 & 1 & $\mathrm{~s}$ & 30 aphyric basalt & 286 & 77 & $\mathrm{P}$ & \\
\hline V1-SDC & Sheeted dikes & Aswad & OM19-35 & No. 55 & 56.29968 & 24.87236 & 2 & $\mathrm{~s}$ & aphyric basalt & 184 & 65 & $\mathrm{P}$ & branch of No. 55 \\
\hline V1-SDC & Sheeted dikes & Aswad & OM19-35 & No. 56 & 56.29968 & 24.87236 & 2 & $\mathrm{~s}$ & 30 aphyric basalt & 292 & 62 & $\mathrm{P}$ & \\
\hline V1-SDC & Sheeted dikes & Aswad & OM19-35 & No. 57 & 56.29968 & 24.87236 & 1 & $\mathrm{~s}$ & $68 \mathrm{cpx}$ basalt & 292 & 62 & $\mathrm{P}$ & \\
\hline V1-SDC & Sheeted dikes & Aswad & OM19-35 & No. 58 & 56.29968 & 24.87236 & 2 & $\mathrm{~s}$ & $99 \mathrm{cpx}$ basalt & 310 & 50 & $\mathrm{P}$ & \\
\hline V1-SDC & Sheeted dikes & Aswad & OM19-35 & No. 59 & 56.29968 & 24.87236 & 2 & $\mathrm{~s}$ & 7 basalt & 307 & 72 & $\mathrm{P}$ & \\
\hline V1-SDC & Sheeted dikes & Aswad & OM19-35 & No. 60 & 56.29968 & 24.87236 & 1 & $\mathrm{~s}$ & 147.5 basalt & 260 & 80 & $\mathrm{P}$ & intruded by No. 61 and 62 \\
\hline V1-SDC & Sheeted dikes & Aswad & OM19-35 & No. 61 & 56.29968 & 24.87236 & 2 & $\mathrm{~s}$ & 5 aphyric basalt & 274 & 77 & $\mathrm{P}$ & \\
\hline V1-SDC & Sheeted dikes & Aswad & OM19-35 & No. 62 & 56.29968 & 24.87236 & 2 & $\mathrm{~s}$ & 3.5 aphyric basalt & 278 & 75 & $\mathrm{P}$ & w/calsic vein \\
\hline V1-SDC & Sheeted dikes & Aswad & OM19-35 & No. 63 & 56.29968 & 24.87236 & 2 & $\mathrm{~s}$ & 42 ol basalt & 284 & 82 & $\mathrm{P}$ & \\
\hline V1-SDC & Sheeted dikes & Aswad & OM19-35 & No. 64 & 56.29968 & 24.87236 & 2 & $\mathrm{~s}$ & $29 \mathrm{cpx}$ basalt & 300 & 72 & $\mathrm{P}$ & \\
\hline V1-SDC & Sheeted dikes & Aswad & OM19-35 & No. 65 & 56.29968 & 24.87236 & 1 & $\mathrm{~s}$ & 15 aphyric basalt & 300 & 72 & $\mathrm{P}$ & \\
\hline V1-SDC & Sheeted dikes & Aswad & OM19-35 & No. 66 & 56.29968 & 24.87236 & 2 & $\mathrm{~s}$ & $43 \mathrm{cpx}$ basalt & 298 & 61 & $\mathrm{P}$ & \\
\hline V1-SDC & Sheeted dikes & Aswad & OM19-35 & No. 67 & 56.29968 & 24.87236 & 2 & $\mathrm{~s}$ & $96 \mathrm{pl} \mathrm{basalt}$ & 280 & 57 & $\mathrm{P}$ & \\
\hline V1-SDC & Sheeted dikes & Aswad & OM19-35 & No. 68 & 56.29968 & 24.87236 & 2 & s & $10 \mathrm{cpx}$ ol basalt & 82 & 78 & $\mathrm{P}$ & \\
\hline V1-SDC & Sheeted dikes & Aswad & OM19-35 & No. 69 & 56.29968 & 24.87236 & 1 & $\mathrm{~s}$ & 107 ol basalt & 92 & 82 & $\mathrm{P}$ & \\
\hline V1-SDC & Sheeted dikes & Aswad & OM19-35 & No. 70 & 56.29968 & 24.87236 & 1 & $\mathrm{~s}$ & $119 \mathrm{cpx}$ basalt & 86 & 80 & $\mathrm{P}$ & \\
\hline V1-SDC & Sheeted dikes & Aswad & OM19-35 & No. 71 & 56.29968 & 24.87236 & 2 & $\mathrm{~s}$ & 33 aphyric basalt & 97 & 89 & $\mathrm{P}$ & \\
\hline V1-SDC & Sheeted dikes & Aswad & OM19-35 & No. 72 & 56.29968 & 24.87236 & & & 19 aphyric basalt & & & & screen \\
\hline V1-SDC & Sheeted dikes & Aswad & OM19-35 & No. 73 & 56.29968 & 24.87236 & 1 & $\mathrm{~s}$ & $124 \mathrm{cpx}$ basalt & 104 & 90 & $\mathrm{P}$ & \\
\hline V1-SDC & Sheeted dikes & Aswad & OM19-35 & No. 74 & 56.29968 & 24.87236 & 2 & $\mathrm{~s}$ & 10 ol basalt & 266 & 76 & $\mathrm{P}$ & \\
\hline V1-SDC & Sheeted dikes & Aswad & OM19-35 & No. 75 & 56.29968 & 24.87236 & 2 & $\mathrm{~s}$ & $14 \mathrm{cpx}$ ol basalt & 270 & 80 & $\mathrm{P}$ & \\
\hline V1-SDC & Sheeted dikes & Aswad & OM19-35 & No. 76 & 56.29968 & 24.87236 & 2 & s & $34 \mathrm{cpx}$ basalt & 279 & 75 & $\mathrm{P}$ & \\
\hline V1-SDC & Sheeted dikes & Aswad & OM19-35 & No. 77 & 56.29968 & 24.87236 & & & 7 aphyric basalt & & & & screen \\
\hline V1-SDC & Sheeted dikes & Aswad & OM19-35 & No. 78 & 56.29968 & 24.87236 & 2 & $\mathrm{~s}$ & 44 aphyric basalt & 280 & 72 & $\mathrm{P}$ & \\
\hline V1-SDC & Sheeted dikes & Aswad & OM19-35 & No. 79 & 56.29968 & 24.87236 & 2 & $\mathrm{~s}$ & 2 aphyric basalt & 282 & 74 & $\mathrm{P}$ & \\
\hline V1-SDC & Sheeted dikes & Aswad & OM19-35 & No. 80 & 56.29968 & 24.87236 & 1 & $\mathrm{~s}$ & 10 aphyric basalt & 282 & 74 & $\mathrm{P}$ & \\
\hline V1-SDC & Sheeted dikes & Aswad & OM19-35 & No. 81 & 56.29998 & 24.87231 & 2 & $\mathrm{~s}$ & $96 \mathrm{pl} \mathrm{basalt}$ & 98 & 87 & $\mathrm{P}$ & Sample \\
\hline V1-SDC & Sheeted dikes & Aswad & OM19-35 & No. 82 & 56.29998 & 24.87231 & 2 & $\mathrm{~s}$ & $46 \mathrm{cpx}$ basalt & 60 & 50 & $\mathrm{P}$ & \\
\hline V1-SDC & Sheeted dikes & Aswad & OM19-35 & No. 83 & 56.29998 & 24.87231 & 2 & $\mathrm{~s}$ & aphyric basalt & 56 & 48 & $\mathrm{P}$ & \\
\hline V1-SDC & Sheeted dikes & Aswad & OM19-35 & No. 84 & 56.29998 & 24.87231 & 2 & B & 4.5 aphyric basalt & 73 & 80 & $\mathrm{P}$ & banding $1,0.5,0.5,1,1 \mathrm{~cm}$ west to east \\
\hline V1-SDC & Sheeted dikes & Aswad & OM19-35 & No. 85 & 56.29998 & 24.87231 & 2 & $\mathrm{~s}$ & 38 aphyric basalt & 78 & 82 & $\mathrm{P}$ & \\
\hline V1-SDC & Sheeted dikes & Aswad & OM19-35 & No. 86 & 56.29998 & 24.87231 & 2 & $\mathrm{~s}$ & 29 aphyric basalt & 284 & 70 & $\mathrm{P}$ & \\
\hline V1-SDC & Sheeted dikes & Aswad & OM19-35 & No. $86^{\prime}$ & 56.29998 & 24.87231 & & & 11 aphyric basalt & & & & screen \\
\hline V1-SDC & Sheeted dikes & Aswad & OM19-35 & No. 87 & 56.29998 & 24.87231 & 2 & $\mathrm{~s}$ & 9 aphyric basalt & 110 & 73 & $\mathrm{P}$ & \\
\hline
\end{tabular}




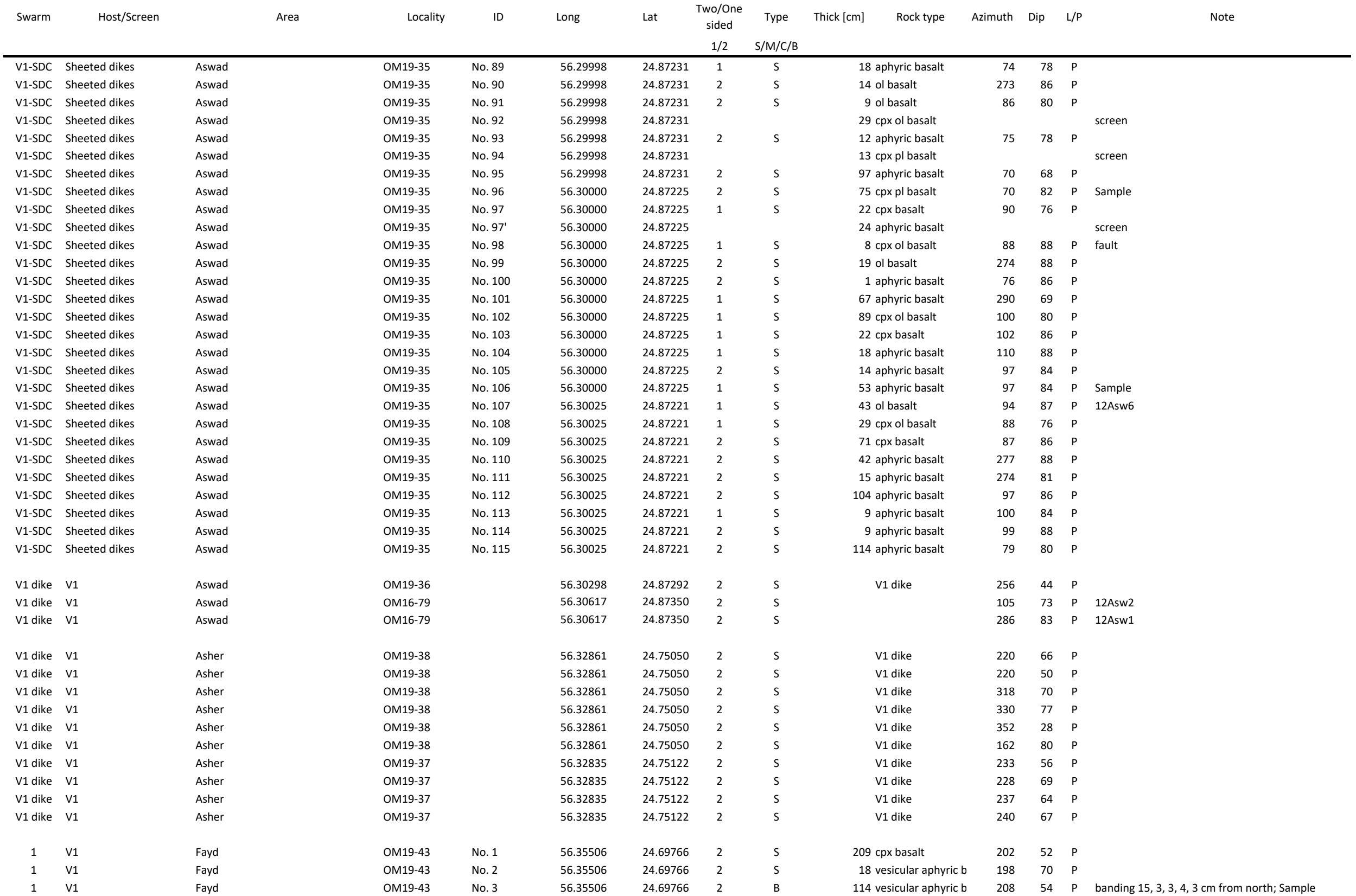




\begin{tabular}{|c|c|c|c|c|c|c|c|c|c|c|c|c|c|}
\hline \multirow[t]{2}{*}{ Swarm } & \multirow[t]{2}{*}{ Host/Screen } & \multirow[t]{2}{*}{ Area } & \multirow[t]{2}{*}{ Locality } & \multirow[t]{2}{*}{ ID } & \multirow[t]{2}{*}{ Long } & \multirow[t]{2}{*}{ Lat } & \multirow{2}{*}{$\begin{array}{c}\text { Two/One } \\
\text { sided } \\
1 / 2 \\
\end{array}$} & \multirow{2}{*}{$\begin{array}{c}\text { Type } \\
S / M / C / B\end{array}$} & \multirow[t]{2}{*}{ Thick [cm] } & \multirow[t]{2}{*}{ Azimuth } & \multirow[t]{2}{*}{ Dip } & \multirow[t]{2}{*}{$L / P$} & \multirow[t]{2}{*}{ Note } \\
\hline & & & & & & & & & & & & & \\
\hline 1 & $\mathrm{~V} 1$ & Fayd & OM19-43 & No. 4 & 56.35506 & 24.69766 & 2 & $\mathrm{~s}$ & 154 vesicular aphyric b & 224 & 78 & $P$ & \\
\hline 1 & $\mathrm{~V} 1$ & Fayd & OM19-43 & No. 5 & 56.35506 & 24.69766 & 2 & $\mathrm{~s}$ & $38 \mathrm{cpx}$ basalt & 210 & 69 & $\mathrm{P}$ & \\
\hline 1 & $\mathrm{v} 1$ & Fayd & OM19-43 & No. 6 & 56.35506 & 24.69766 & 2 & $\mathrm{~s}$ & 50 aphyric basalt & 234 & 64 & $\mathrm{P}$ & 4 drill holes \\
\hline 1 & v1 & Fayd & OM19-43 & No. 7 & 56.35506 & 24.69766 & 2 & $\mathrm{~s}$ & 40 aphyric basalt & 200 & 62 & $\mathrm{P}$ & \\
\hline 1 & $\mathrm{v} 1$ & Fayd & OM19-43 & No. 8 & 56.35506 & 24.69766 & 2 & $\mathrm{~s}$ & 234 aphyric basalt & 212 & 52 & $\mathrm{P}$ & \\
\hline 1 & v1 & Fayd & OM19-43 & No. 8 & 56.35506 & 24.69766 & 2 & $\mathrm{~s}$ & 234 aphyric basalt & 232 & 38 & $\mathrm{P}$ & south side of No. 8 \\
\hline 1 & v1 & Fayd & OM19-43 & No. 9 & 56.35506 & 24.69766 & 1 & $\mathrm{~s}$ & $87 \mathrm{cpx}$ basalt & 234 & 51 & $\mathrm{P}$ & \\
\hline 1 & $\mathrm{v} 1$ & Fayd & OM19-43 & No. 10 & 56.35506 & 24.69766 & 2 & $\mathrm{~s}$ & 89 aphyric basalt & 220 & 74 & $\mathrm{P}$ & variole rich in chilled margin; Sample \\
\hline 1 & v1 & Fayd & OM19-43 & No. 10 & 56.35506 & 24.69766 & 2 & $\mathrm{~s}$ & 89 aphyric basalt & 172 & 70 & $\mathrm{P}$ & bended \\
\hline 1 & v1 & Fayd & OM19-43 & No. 11 & 56.35506 & 24.69766 & 2 & $\mathrm{~s}$ & $78 \mathrm{cpx}$ basalt & 210 & 50 & $\mathrm{P}$ & \\
\hline 1 & Sheeted dikes & Fayd & OM19-46 & No. 1 & 56.32424 & 24.71875 & 1 & $\mathrm{~s}$ & aphyric basalt & 182 & 82 & $\mathrm{P}$ & \\
\hline 1 & Sheeted dikes & Fayd & OM19-46 & No. 2 & 56.32424 & 24.71875 & 2 & $\mathrm{~s}$ & aphyric basalt & 164 & 88 & $\mathrm{P}$ & \\
\hline 1 & Sheeted dikes & Fayd & OM19-46 & No. 3 & 56.32424 & 24.71875 & 1 & $\mathrm{~s}$ & aphyric basalt & 168 & 90 & $\mathrm{P}$ & \\
\hline 1 & Sheeted dikes & Fayd & OM19-46 & No. 4 & 56.32424 & 24.71875 & 2 & $\mathrm{~s}$ & aphyric basalt & 180 & 87 & $\mathrm{P}$ & \\
\hline 1 & Sheeted dikes & Fayd & OM19-46 & No. 5 & 56.32424 & 24.71875 & 2 & $\mathrm{~s}$ & aphyric basalt & 170 & 84 & $\mathrm{P}$ & \\
\hline 1 & Sheeted dikes & Fayd & OM19-46 & No. 6 & 56.32424 & 24.71875 & 1 & $\mathrm{~s}$ & aphyric basalt & 164 & 85 & $\mathrm{P}$ & \\
\hline 1 & Sheeted dikes & Fayd & OM19-46 & No. 7 & 56.32424 & 24.71875 & 2 & $\mathrm{~s}$ & aphyric basalt & 168 & 84 & $\mathrm{P}$ & \\
\hline 1 & Sheeted dikes & Fayd & OM19-46 & No. 8 & 56.32424 & 24.71875 & 2 & s & aphyric dolerite & 168 & 87 & $\mathrm{P}$ & \\
\hline 1 & Sheeted dikes & Fayd & OM19-46 & No. 9 & 56.32424 & 24.71875 & 2 & $\mathrm{~s}$ & aphyric basalt & 172 & 86 & $\mathrm{P}$ & \\
\hline 1 & Sheeted dikes & Fayd & OM19-46 & No. 10 & 56.32424 & 24.71875 & 1 & s & aphyric basalt & 172 & 88 & $\mathrm{P}$ & \\
\hline 1 & Sheeted dikes & Fayd & OM19-46 & No. 1 & 56.32424 & 24.71875 & 2 & $\mathrm{~s}$ & aphyric basalt & 162 & 80 & $\mathrm{P}$ & \\
\hline 1 & Sheeted dikes & Fayd & OM19-46 & No. 2 & 56.32424 & 24.71875 & 2 & $\mathrm{~s}$ & aphyric basalt & 160 & 79 & $\mathrm{P}$ & \\
\hline 1 & Sheeted dikes & Fayd & OM19-46 & No. 3 & 56.32424 & 24.71875 & 1 & $\mathrm{~s}$ & aphyric dolerite & 172 & 84 & $\mathrm{P}$ & \\
\hline 1 & Sheeted dikes & Fayd & OM19-46 & No. 4 & 56.32424 & 24.71875 & 1 & $\mathrm{~s}$ & porphyritic dolerit & 176 & 84 & $\mathrm{P}$ & \\
\hline 1 & Sheeted dikes & Fayd & OM19-46 & No. 5 & 56.32424 & 24.71875 & 2 & $\mathrm{~s}$ & & 162 & 80 & $\mathrm{P}$ & \\
\hline 1 & Sheeted dikes & Fayd & OM19-46 & No. 6 & 56.32424 & 24.71875 & 1 & $\mathrm{~s}$ & porphyritic basalt & 166 & 90 & $\mathrm{P}$ & \\
\hline 1 & Sheeted dikes & Fayd & OM19-46 & No. 7 & 56.32424 & 24.71875 & 2 & $\mathrm{~s}$ & aphyric dolerite & 176 & 88 & $\mathrm{P}$ & \\
\hline 1 & Sheeted dikes & Fayd & OM19-46 & No. 8 & 56.32424 & 24.71875 & 2 & $\mathrm{~s}$ & aphyric dolerite & 358 & 88 & $\mathrm{P}$ & \\
\hline 1 & Sheeted dikes & Fayd & OM19-46 & No. 9 & 56.32424 & 24.71875 & 2 & $\mathrm{~s}$ & aphyric basalt & 178 & 88 & $\mathrm{P}$ & \\
\hline 1 & Sheeted dikes & Fayd & OM19-46 & No. 10 & 56.32424 & 24.71875 & 2 & $\mathrm{~s}$ & aphyric basalt & 348 & 86 & $\mathrm{P}$ & \\
\hline 1 & Sheeted dikes & Fayd & OM19-46 & No. 11 & 56.32424 & 24.71875 & 2 & $\mathrm{~s}$ & aphyric basalt & 180 & 84 & $\mathrm{P}$ & \\
\hline 1 & Sheeted dikes & Fayd & OM19-46 & No. 12 & 56.32424 & 24.71875 & 1 & $\mathrm{~s}$ & aphyric basalt & 170 & 86 & $\mathrm{P}$ & \\
\hline 1 & Sheeted dikes & Fayd & OM19-46 & No. 13 & 56.32424 & 24.71875 & 1 & $\mathrm{~s}$ & aphyric basalt & 176 & 84 & $\mathrm{P}$ & \\
\hline 1 & Sheeted dikes & Fayd & OM19-46 & No. 14 & 56.32424 & 24.71875 & 2 & s & & 166 & 90 & $\mathrm{P}$ & \\
\hline 1 & Sheeted dikes & Fayd & OM19-46 & No. 15 & 56.32424 & 24.71875 & 1 & s & aphyric basalt & 170 & 88 & $\mathrm{P}$ & \\
\hline 1 & Sheeted dikes & Fayd & OM19-45 & No. $1^{\prime}$ & 56.32298 & 24.71308 & 2 & $\mathrm{~s}$ & 18 dacite & 19 & 79 & $\mathrm{P}$ & bended No. 1' \\
\hline 1 & Sheeted dikes & Fayd & OM19-45 & No. 26 & 56.32298 & 24.71308 & 1 & s & 60 ol pyroxenite & 34 & 88 & $\mathrm{P}$ & cut No. 25 and 29 \\
\hline 1 & Sheeted dikes & Fayd & OM19-45 & No. 27 & 56.32298 & 24.71308 & 1 & $\mathrm{~s}$ & 40 ol pyroxenite & 35 & 87 & $\mathrm{P}$ & cut No. 26 \\
\hline 1 & Sheeted dikes & Fayd & OM19-45 & No. 24 & 56.32298 & 24.71308 & 1 & $\mathrm{~s}$ & 60 ol pyroxenite & 46 & 87 & $\mathrm{P}$ & \\
\hline 1 & Sheeted dikes & Fayd & OM19-45 & No. 2 & 56.32298 & 24.71308 & 2 & $\mathrm{~s}$ & 28 clinopyroxenite? & 60 & 78 & $\mathrm{P}$ & \\
\hline 1 & Sheeted dikes & Fayd & OM19-45 & No. 0 & 56.32298 & 24.71308 & 1 & & pyroxenite & 68 & 82 & $\mathrm{P}$ & \\
\hline 1 & Sheeted dikes & Fayd & OM19-45 & No. 6 & 56.32298 & 24.71308 & 2 & $\mathrm{~s}$ & 6 pyroxenite & 76 & 76 & $P$ & \\
\hline 1 & Sheeted dikes & Fayd & OM19-45 & No. 20 & 56.32298 & 24.71308 & 2 & $\mathrm{~s}$ & 2.5 ol pyroxenite & 79 & 13 & $\mathrm{P}$ & bended \\
\hline 1 & Sheeted dikes & Fayd & OM19-45 & No. 28 & 56.32298 & 24.71308 & 2 & $\mathrm{~s}$ & 8 diolite? & 218 & 59 & $\mathrm{P}$ & intrude into No. 31 \\
\hline 1 & Sheeted dikes & Fayd & OM19-45 & No. 10 & 56.32298 & 24.71308 & 2 & $\mathrm{~s}$ & 71 ol pyroxenite & 222 & 63 & $\mathrm{P}$ & \\
\hline
\end{tabular}




\begin{tabular}{|c|c|c|c|c|c|c|c|c|c|c|c|c|c|}
\hline \multirow[t]{2}{*}{ Swarm } & \multirow[t]{2}{*}{ Host/Screen } & \multirow[t]{2}{*}{ Area } & \multirow[t]{2}{*}{ Locality } & \multirow[t]{2}{*}{ ID } & \multirow[t]{2}{*}{ Long } & \multirow[t]{2}{*}{ Lat } & \multirow{2}{*}{$\begin{array}{c}\text { Two/One } \\
\text { sided } \\
1 / 2\end{array}$} & \multirow{2}{*}{$\begin{array}{c}\text { Type } \\
\text { S/M/C/B }\end{array}$} & \multirow[t]{2}{*}{ Thick [cm] } & \multirow[t]{2}{*}{ Azimuth } & \multirow[t]{2}{*}{ Dip } & \multirow[t]{2}{*}{$L / P$} & \multirow[t]{2}{*}{ Note } \\
\hline & & & & & & & & & & & & & \\
\hline 1 & Sheeted dikes & Fayd & OM19-45 & No. 33 & 56.32298 & 24.71308 & 1 & $\mathrm{~s}$ & 14 ol pyroxenite & 226 & 60 & $\mathrm{P}$ & \\
\hline 1 & Sheeted dikes & Fayd & OM19-45 & No. $1^{\prime}$ & 56.32298 & 24.71308 & 2 & $\mathrm{~s}$ & 33 dacite & 228 & 58 & $\mathrm{P}$ & branch of No. 1; Sample \\
\hline 1 & Sheeted dikes & Fayd & OM19-45 & No. 9 & 56.32298 & 24.71308 & 2 & B & 66 ol pyroxenite & 228 & 43 & $\mathrm{P}$ & western side of No. 9 \\
\hline 1 & Sheeted dikes & Fayd & OM19-45 & No. 21 & 56.32298 & 24.71308 & 1 & $\mathrm{~s}$ & 16 pl-bearing ol pyrox & 230 & 50 & $\mathrm{P}$ & \\
\hline 1 & Sheeted dikes & Fayd & OM19-45 & No. 34 & 56.32298 & 24.71308 & 2 & $\mathrm{~s}$ & 33 pl-bearing ol pyrox & 232 & 57 & $\mathrm{P}$ & \\
\hline 1 & Sheeted dikes & Fayd & OM19-45 & No. 43 & 56.32298 & 24.71308 & & & gabbronorite & 237 & 70 & $\mathrm{P}$ & \\
\hline 1 & Sheeted dikes & Fayd & OM19-45 & No. 22 & 56.32298 & 24.71308 & 2 & $\mathrm{~s}$ & 3 pyroxenite & 240 & 48 & $\mathrm{P}$ & \\
\hline 1 & Sheeted dikes & Fayd & OM19-45 & No. 37 & 56.32298 & 24.71308 & 2 & $\mathrm{~s}$ & 68 ol pyroxenite & 240 & 71 & $\mathrm{P}$ & cut No. $38,39,40,41$ \\
\hline 1 & Sheeted dikes & Fayd & OM19-45 & No. 20 & 56.32298 & 24.71308 & 2 & $\mathrm{~s}$ & 4 ol pyroxenite & 242 & 74 & $\mathrm{P}$ & \\
\hline 1 & Sheeted dikes & Fayd & OM19-45 & No. 23 & 56.32298 & 24.71308 & 1 & $\mathrm{~s}$ & 70 pyroxenite & 242 & 61 & $\mathrm{P}$ & \\
\hline 1 & Sheeted dikes & Fayd & OM19-45 & No. 36 & 56.32298 & 24.71308 & 2 & $\mathrm{~s}$ & 122 pl-bearing ol pyrox & 242 & 71 & $\mathrm{P}$ & \\
\hline 1 & Sheeted dikes & Fayd & OM19-45 & No. 32 & 56.32298 & 24.71308 & 1 & $\mathrm{~s}$ & 184 ol gabbronorite & 243 & 61 & $\mathrm{P}$ & \\
\hline 1 & Sheeted dikes & Fayd & OM19-45 & No. 8 & 56.32298 & 24.71308 & 1 & $\mathrm{~s}$ & 64 ol pyroxenite & 250 & 58 & $\mathrm{P}$ & \\
\hline 1 & Sheeted dikes & Fayd & OM19-45 & No. 35 & 56.32298 & 24.71308 & 2 & $\mathrm{~s}$ & 36 pl-bearing ol pyrox & 254 & 63 & $\mathrm{P}$ & \\
\hline 1 & Sheeted dikes & Fayd & OM19-45 & No. 5 & 56.32298 & 24.71308 & 2 & $\mathrm{~s}$ & 17 gabbronorite & 98 & 69 & $\mathrm{P}$ & pegmatitic \\
\hline 1 & Sheeted dikes & Fayd & OM19-45 & No. 7 & 56.32298 & 24.71308 & 2 & $\mathrm{~s}$ & 84 ol pyroxenite & 100 & 82 & $\mathrm{P}$ & \\
\hline 1 & Sheeted dikes & Fayd & OM19-45 & No. 4 & 56.32298 & 24.71308 & 2 & $\mathrm{~s}$ & 48 gabbronorite & 102 & 76 & $\mathrm{P}$ & echelon \\
\hline 1 & Sheeted dikes & Fayd & OM19-45 & No. 11 & 56.32298 & 24.71308 & 2 & $\mathrm{~s}$ & 26 ol pyroxenite & 102 & 72 & $\mathrm{P}$ & \\
\hline 1 & Sheeted dikes & Fayd & OM19-45 & No. 14 & 56.32298 & 24.71308 & 2 & $\mathrm{~s}$ & 120 ol gabbronorite & 103 & 77 & $\mathrm{P}$ & \\
\hline 1 & Sheeted dikes & Fayd & OM19-45 & No. 42 & 56.32298 & 24.71308 & 2 & $\mathrm{~s}$ & 60 troctrite? & 106 & 58 & $\mathrm{P}$ & \\
\hline 1 & Sheeted dikes & Fayd & OM19-45 & No. 40 & 56.32298 & 24.71308 & 2 & $\mathrm{~s}$ & $81 \mathrm{pl}$-bearing wehrite & 108 & 74 & $\mathrm{P}$ & \\
\hline 1 & Sheeted dikes & Fayd & OM19-45 & No. 4 & 56.32298 & 24.71308 & 2 & $\mathrm{~s}$ & 30 gabbronorite & 113 & 66 & $\mathrm{P}$ & \\
\hline 1 & Sheeted dikes & Fayd & OM19-45 & No. 12 & 56.32298 & 24.71308 & 1 & $\mathrm{~s}$ & 68 ol pyroxenite & 114 & 62 & $\mathrm{P}$ & Sample \\
\hline 1 & Sheeted dikes & Fayd & OM19-45 & No. 39 & 56.32298 & 24.71308 & 2 & $\mathrm{~s}$ & 123 ol pyroxenite & 121 & 85 & $\mathrm{P}$ & \\
\hline 1 & Sheeted dikes & Fayd & OM19-45 & No. 31 & 56.32298 & 24.71308 & 2 & $\mathrm{~s}$ & $36 \mathrm{pl}$-bearing pyroxer & 123 & 71 & $\mathrm{P}$ & \\
\hline 1 & Sheeted dikes & Fayd & OM19-45 & No. 15 & 56.32298 & 24.71308 & 1 & $\mathrm{~s}$ & 111 ol pyroxenite & 135 & 77 & $\mathrm{P}$ & \\
\hline 1 & Sheeted dikes & Fayd & OM19-45 & No. 29 & 56.32298 & 24.71308 & 1 & $\mathrm{~s}$ & 124 pyroxenite & 142 & 76 & $\mathrm{P}$ & \\
\hline 1 & Sheeted dikes & Fayd & OM19-45 & No. $1^{\prime}$ & 56.32298 & 24.71308 & 2 & $\mathrm{~s}$ & 8 dacite & 276 & 74 & $\mathrm{P}$ & branch of No. 1' \\
\hline 1 & Sheeted dikes & Fayd & OM19-45 & No. 9 & 56.32298 & 24.71308 & 2 & B & 66 ol pyroxenite & 280 & 88 & $\mathrm{P}$ & $15 \mathrm{~cm}$ banding \\
\hline 1 & Sheeted dikes & Fayd & OM19-45 & No. 16 & 56.32298 & 24.71308 & 2 & $\mathrm{~s}$ & 35 & 282 & 52 & $\mathrm{P}$ & \\
\hline 1 & Sheeted dikes & Fayd & OM19-45 & No. 25 & 56.32298 & 24.71308 & 1 & $\mathrm{~s}$ & $133 \mathrm{pl}$-bearing wehrite & 290 & 77 & $\mathrm{P}$ & \\
\hline 1 & Sheeted dikes & Fayd & OM19-45 & No. 33 & 56.32298 & 24.71308 & 1 & $\mathrm{~s}$ & 56 ol pyroxenite & 291 & 79 & $\mathrm{P}$ & bended \\
\hline 1 & Sheeted dikes & Fayd & OM19-45 & No. 1 & 56.32298 & 24.71308 & 2 & $\mathrm{~s}$ & 23 dacite & 296 & 59 & $\mathrm{P}$ & \\
\hline 1 & Sheeted dikes & Fayd & OM19-45 & No. 38 & 56.32298 & 24.71308 & 1 & $\mathrm{~s}$ & $154 \mathrm{pl}$-bearing wehrite & 316 & 78 & $\mathrm{P}$ & \\
\hline 1 & Sheeted dikes & Fayd & OM19-45 & No. 18 & 56.32298 & 24.71308 & 2 & $\mathrm{~s}$ & 20 pyroxenite & 320 & 88 & $\mathrm{P}$ & \\
\hline 1 & Sheeted dikes & Fayd & OM19-45 & No. 3 & 56.32298 & 24.71308 & & & & & & & screen \\
\hline 1 & Sheeted dikes & Fayd & OM19-45 & No. 13 & 56.32298 & 24.71308 & 1 & & ol gabbronorite & & & & \\
\hline 1 & Sheeted dikes & Fayd & OM19-45 & No. 17 & 56.32298 & 24.71308 & & & 124 pyroxenite & & & & screen \\
\hline 1 & Sheeted dikes & Fayd & OM19-45 & No. 19 & 56.32298 & 24.71308 & & & pl-bearing ol pyrox & & & & \\
\hline 1 & Sheeted dikes & Fayd & OM19-45 & No. 30 & 56.32298 & 24.71308 & & & 58 pyroxenite & & & & screen \\
\hline 1 & Sheeted dikes & Fayd & OM19-45 & No. 41 & 56.32298 & 24.71308 & & & 255 troctrite? & & & & \\
\hline 1 & Sheeted dikes & Fayd & OM19-47 & No. 1 & 56.32091 & 24.71845 & 2 & $\mathrm{~s}$ & ol cpx boninite? & 216 & 70 & $\mathrm{P}$ & \\
\hline 1 & Sheeted dikes & Fayd & OM19-47 & No. 2 & 56.32091 & 24.71845 & 2 & $\mathrm{~s}$ & pl cpx andesite? & 210 & 52 & $\mathrm{P}$ & \\
\hline 1 & Sheeted dikes & Fayd & OM19-47 & No. 3 & 56.32091 & 24.71845 & 2 & $\mathrm{~s}$ & 372 ol cpx boninite? & 240 & 68 & $\mathrm{P}$ & \\
\hline 1 & Sheeted dikes & Fayd & OM19-47 & No. 4 & 56.32091 & 24.71845 & 2 & $\mathrm{~s}$ & $118 \mathrm{cpx}$ boninite? & 230 & 50 & $\mathrm{P}$ & \\
\hline 1 & Sheeted dikes & Fayd & OM19-47 & No. 5 & 56.32091 & 24.71845 & 2 & $\mathrm{~s}$ & boninite? & 250 & 64 & $\mathrm{P}$ & \\
\hline
\end{tabular}


$1 / 2 \quad \mathrm{~S} / \mathrm{M} / \mathrm{C} / \mathrm{B}$

$\begin{array}{ll}\text { Sheeted dikes } & \text { Buwaydar W } \\ \text { Sheeted dikes } & \text { Buwaydar W } \\ \text { Sheeted dikes } & \text { Buwaydar W } \\ \text { Sheeted dikes } & \text { Buwaydar W } \\ \text { Sheeted dikes } & \text { Buwaydar W } \\ \text { Sheeted dikes } & \text { Buwaydar W } \\ \text { Sheeted dikes } & \text { Buwaydar W } \\ \text { Sheeted dikes } & \text { Buwaydar W } \\ \text { Sheeted dikes } & \text { Buwaydar W } \\ \text { Sheeted dikes } & \text { Buwaydar W } \\ \text { Sheeted dikes } & \text { Buwaydar W } \\ & \\ \text { Sheeted dikes } & \text { Buwaydar W } \\ \text { Sheeted dikes } & \text { Buwaydar W } \\ \text { Sheeted dikes } & \text { Buwaydar W } \\ \text { Sheeted dikes } & \text { Buwaydar W } \\ \text { Sheeted dikes } & \text { Buwaydar W } \\ \text { Sheeted dikes } & \text { Buwaydar W } \\ \text { Sheeted dikes } & \text { Buwaydar W } \\ \text { Sheeted dikes } & \text { Buwaydar W } \\ \text { Sheeted dikes } & \text { Buwaydar W } \\ \text { Sheeted dikes } & \text { Buwaydar W } \\ \text { Sheeted dikes } & \text { Buwaydar W } \\ \text { Sheeted dikes } & \text { Buwaydar W } \\ \text { Sheeted dikes } & \text { Buwaydar W } \\ \text { Sheeted dikes } & \text { Buwaydar W } \\ \text { Sheeted dikes } & \text { Buwaydar W } \\ \text { Sheeted dikes } & \text { Buwaydar W } \\ & \\ \text { Sheeted dikes } & \text { Rajmi } \\ \text { Sheeted dikes } & \text { Rajmi } \\ \text { Sheeted dikes } & \text { Rajmi } \\ \text { Sheeted dikes } & \text { Rajmi } \\ \text { Sheeted dikes } & \text { Rajmi } \\ \text { Sheeted dikes } & \text { Rajmi } \\ \text { Sheeted dikes } & \text { Rajmi } \\ \text { Sheeted dikes } & \text { Rajmi } \\ \text { Sheeted dikes } & \text { Rajmi } \\ \text { Sheeted dikes } & \text { Rajmi } \\ \text { Sheeted dikes } & \text { Rajmi } \\ \text { Sheeted dikes } & \text { Rajmi } \\ \text { Sheeted dikes } & \text { Rajmi } \\ \text { Sheeted dikes } & \text { Rajmi } \\ & \\ & \end{array}$

\section{M11-30}

OM11-30

OM11-30

OM11-30

OM11-30

OM11-30

OM11-30

OM11-30

M11-30

OM11-31

OM11-31

OM11-31

OM11-31

OM11-31

OM11-31

OM11-31

OM11-31

OM11-31

OM11-31

OM11-31

M11-31

OM11-31
OM11-31

\section{No. 1}

No. 2

No. 3

No. 4

No. 5

No. 6

No. 8

No. 9

No. 10

No. 11

No. 1

No. 2

No. 3

No. 4

No. 5

No. 7

No. 7

No. 8

No. 9
No. 10

No. 11

No. 12

No. 13

No. 14

No. 15

\section{OM19-23}

OM19-23

OM19-23

DM19-23 No. 2

OM19-23 No. 3

OM19-23 No. 4

No. 6

OM19-23 No. 7

NoM19-23 No. 7

No. 8

No. 9

$\begin{array}{ll}\text { OM19-23 } & \text { No. } 10 \\ \text { OM19-23 } & \text { No. } 10\end{array}$

No. 10
$56.35302778 \quad 24.66580556$

$\begin{array}{ll}56.35302778 & 24.66580556\end{array}$

$56.35302778 \quad 24.66580556$

$56.35302778 \quad 24.66580556$

$56.35302778 \quad 24.66580556$

$56.35302778 \quad 24.66580556$

$56.35302778 \quad 24.66580556$

$56.35302778 \quad 24.66580556$

$56.35302778 \quad 24.66580556$

$56.35302778 \quad 24.66580556$

$56.35302778 \quad 24.66580556$

$56.34761111 \quad 24.66536111$

$56.34761111 \quad 24.66536111$

$56.34761111 \quad 24.66536111$

$56.34761111 \quad 24.66536111$

$56.34761111 \quad 24.66536111$

$56.34761111 \quad 24.66536111$

$56.34761111 \quad 24.66536111$

$56.34761111 \quad 24.66536111$

$\begin{array}{ll}56.34761111 & 24.66536111\end{array}$

$56.34761111 \quad 24.66536111$

$56.34761111 \quad 24.66536111$

$56.34761111 \quad 24.66536111$

$56.34761111 \quad 24.66536111$

$56.34761111 \quad 24.66536111$

$56.34761111 \quad 24.66536111$

$56.34670 \quad 24.62985$

\subsection{0
-24.62985}

$56.34670 \quad 24.62985$

$\begin{array}{ll}56.34670 & 24.62985 \\ 56.34670 & 24.62985\end{array}$

$\begin{array}{ll}56.34670 & 24.62985\end{array}$

$56.34670 \quad 24.62985$

$56.34674 \quad 24.62944$

$56.34670 \quad 24.62985$

$56.34670 \quad 24.62985$

$56.34671 \quad 24.62947$
56.34671

$\begin{array}{ll}56.34671 & 24.62947\end{array}$

$\begin{array}{ll}56.34671 & 24.62947 \\ 56.34671 & 24.62947\end{array}$

Rajmi OM19-23

dolerite

dolerite

cpx basalt

porphyritic

aphyric basalt

porphyritic

porphyritic

$278 \quad 40 \quad \mathrm{P}$

$292 \quad 50 \quad \mathrm{P}$

$\begin{array}{lll}280 & 55 & \mathrm{P}\end{array}$

$286 \quad 51 \quad \mathrm{P}$

$\begin{array}{lll}300 & 60 & \mathrm{P}\end{array}$

$\begin{array}{lll}288 & 66 & \mathrm{P}\end{array}$

$\begin{array}{lll}286 & 61 \quad \mathrm{P} \\ 240 & 29 & -\end{array}$

$\begin{array}{lll}240 & 29 & \mathrm{P}\end{array}$

$\begin{array}{lll}236 & 28 & \mathrm{P}\end{array}$

$\begin{array}{lll}172 & 74 & P\end{array}$

$\begin{array}{lll}162 & 75 & P\end{array}$

$\begin{array}{lll}190 & 54 & \mathrm{P}\end{array}$

$\begin{array}{lll}182 & 48 & \mathrm{P}\end{array}$

$\begin{array}{lll}196 & 49 & \mathrm{P}\end{array}$

$194 \quad 56 \quad \mathrm{P}$

$\begin{array}{lll}180 \quad 52 & \mathrm{P}\end{array}$

$\begin{array}{lll}186 & 74 & \mathrm{P}\end{array}$

$\begin{array}{lll}185 & 78 & \mathrm{P}\end{array}$

$\begin{array}{lll}226 & 53 & \mathrm{P}\end{array}$

$\begin{array}{lll}227 & 44 & \mathrm{P}\end{array}$

$\begin{array}{lll}168 & 46 & \mathrm{P}\end{array}$

$\begin{array}{lll}192 & 77 & P\end{array}$

$\begin{array}{lll}174 & 55 & \mathrm{P}\end{array}$

$\begin{array}{lll}183 & 44 & P\end{array}$

$\begin{array}{lll}197 & 64 & \mathrm{P}\end{array}$

$\begin{array}{lll}174 & 53 & \mathrm{P}\end{array}$

$185 \quad 66 \quad P$

$\begin{array}{llll}204 & 65 & \mathrm{P} & \text { north side near chilled margin }\end{array}$

$\begin{array}{lll}180 & 59 & \mathrm{P}\end{array}$

$\begin{array}{lll}193 & 63 & \mathrm{P} \\ 200 & 63 & \mathrm{P}\end{array}$

$\begin{array}{lll}200 & 63 & \mathrm{P}\end{array}$

$202 \quad 65 \quad \mathrm{P}$

$\begin{array}{lll}174 & 56 & \mathrm{P} \\ 168 & 58 & \mathrm{P}\end{array}$

$168 \quad 58 \quad \mathrm{P}$

aphyric basat

$\begin{array}{llll}180 & 58 \quad \mathrm{P} & \text { north side near chiled margin }\end{array}$

porphyritic

$166 \quad 63 \quad P$ screen

porphyritic

$\begin{array}{lll}166 & 63 & \mathrm{P} \\ 176 & 70 & \mathrm{P}\end{array}$

$\begin{array}{lllll}\text { porphyrtic } & 176 & 70 & \mathrm{P} & \text { north side near chilled margin }\end{array}$ 


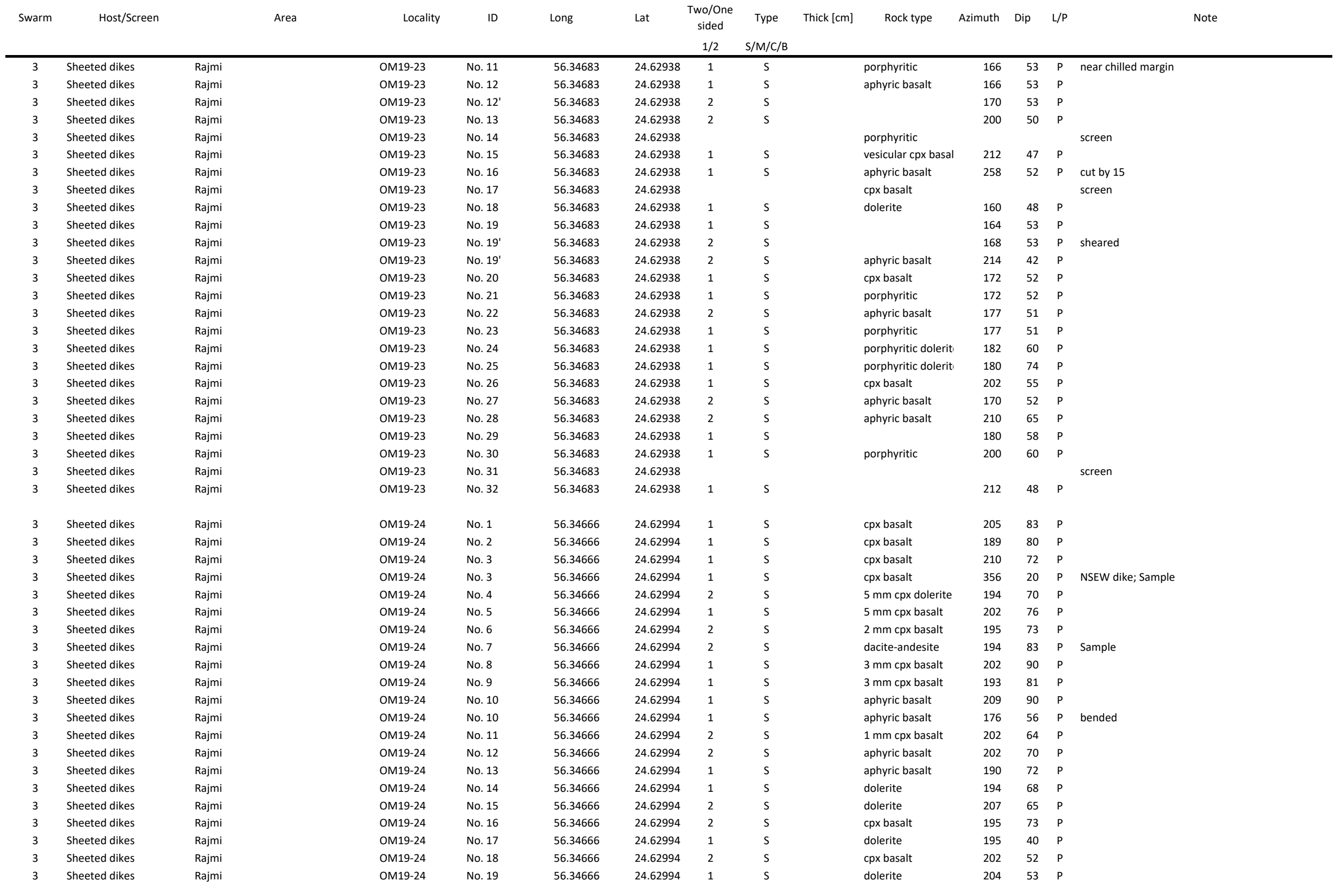




\begin{tabular}{|c|c|c|c|c|c|c|c|c|c|c|c|c|c|c|c|}
\hline \multirow[t]{2}{*}{ Swarm } & \multirow[t]{2}{*}{ Host/Screen } & \multirow{2}{*}{\multicolumn{2}{|c|}{ Area }} & \multirow[t]{2}{*}{ Locality } & \multirow[t]{2}{*}{ ID } & \multirow[t]{2}{*}{ Long } & \multirow[t]{2}{*}{ Lat } & \multirow{2}{*}{$\begin{array}{c}\text { Two/One } \\
\text { sided } \\
1 / 2\end{array}$} & \multirow{2}{*}{$\begin{array}{c}\text { Type } \\
\mathrm{S} / \mathrm{M} / \mathrm{C} / \mathrm{B}\end{array}$} & \multirow[t]{2}{*}{ Thick [cm] } & \multirow[t]{2}{*}{ Rock type } & \multirow[t]{2}{*}{ Azimuth } & \multirow[t]{2}{*}{ Dip } & \multirow[t]{2}{*}{$L / P$} & \multirow[t]{2}{*}{ Note } \\
\hline & & & & & & & & & & & & & & & \\
\hline 3 & Sheeted dikes & Rajmi & & OM19-24 & No. 20 & 56.34666 & 24.62994 & 2 & $\mathrm{~s}$ & & cpx pl basalt & 194 & 60 & $P$ & Sample \\
\hline 3 & Sheeted dikes & Rajmi & & OM19-24 & No. 21 & 56.34666 & 24.62994 & 2 & s & & dolerite & 202 & 53 & $\mathrm{P}$ & \\
\hline 3 & Sheeted dikes & Rajmi & & OM19-24 & No. 22 & 56.34666 & 24.62994 & 2 & $\mathrm{~s}$ & & aphyric basalt & 194 & 62 & $\mathrm{P}$ & \\
\hline 3 & Sheeted dikes & Rajmi & & OM19-24 & No. 23 & 56.34666 & 24.62994 & 1 & $\mathrm{~s}$ & & $1 \mathrm{~mm}$ cpx basalt & 202 & 80 & $\mathrm{P}$ & \\
\hline 3 & Sheeted dikes & Rajmi & & OM19-24 & No. 24 & 56.34666 & 24.62994 & 1 & s & & dolerite & 185 & 66 & $\mathrm{P}$ & \\
\hline 3 & Sheeted dikes & Rajmi & & OM19-24 & No. 25 & 56.34666 & 24.62994 & 1 & s & & $1 \mathrm{~mm}$ cpx basalt & 192 & 68 & $\mathrm{P}$ & \\
\hline 3 & Sheeted dikes & Rajmi & & OM19-24 & No. 26 & 56.34666 & 24.62994 & 1 & s & & $0.5 \mathrm{~mm}$ cp $\times$ basalt & 210 & 38 & $\mathrm{P}$ & sheared \\
\hline 3 & Sheeted dikes & Rajmi & & OM19-24 & No. 27 & 56.34666 & 24.62994 & 1 & $\mathrm{~s}$ & & $1 \mathrm{~mm}$ cpx basalt & 206 & 44 & $\mathrm{P}$ & \\
\hline 3 & Sheeted dikes & Rajmi & & OM19-24 & No. 28 & 56.34666 & 24.62994 & 2 & s & & $0.5 \mathrm{~mm}$ cp $\times$ basalt & 204 & 45 & $\mathrm{P}$ & \\
\hline 3 & Sheeted dikes & Rajmi & & OM19-24 & No. 29 & 56.34666 & 24.62994 & 1 & $\mathrm{~s}$ & & cpx pl basalt & 166 & 80 & $\mathrm{P}$ & \\
\hline 3 & Sheeted dikes & Rajmi & & OM19-24 & No. 30 & 56.34666 & 24.62994 & 1 & $\mathrm{~s}$ & & $5 \mathrm{~mm}$ cp x basalt & 200 & 69 & $\mathrm{P}$ & $\max 2 \mathrm{~cm} \mathrm{cpx}$ \\
\hline 3 & gabbros & Rajmi & & OM19-25 & No. 0 & 56.33172 & 24.62788 & & & & Gabbro & & & & \\
\hline 3 & gabbros & Rajmi & & OM19-25 & No. 1 & 56.33172 & 24.62788 & 2 & s & & $3 \mathrm{cpx}$ phyric & 190 & 88 & $\mathrm{P}$ & \\
\hline 3 & gabbros & Rajmi & & OM19-25 & No. 2 & 56.33172 & 24.62788 & 2 & $\mathrm{~s}$ & & 8 aphyric & 18 & 80 & $\mathrm{P}$ & \\
\hline 3 & gabbros & Rajmi & & OM19-25 & No. 3 & 56.33172 & 24.62788 & 2 & $\mathrm{~s}$ & & 6 porphyritic & 180 & 67 & $\mathrm{P}$ & $\max 5$ mm cpx; Sample \\
\hline 3 & gabbros & Rajmi & & OM19-25 & No. 4 & 56.33172 & 24.62788 & 2 & $\mathrm{~s}$ & & 0 dolerite & 190 & 75 & $\mathrm{P}$ & \\
\hline 3 & gabbros & Rajmi & & OM19-25 & No. 5 & 56.33172 & 24.62788 & 2 & $\mathrm{~s}$ & & 9 porphyritic & 192 & 70 & $\mathrm{P}$ & $\max 5 \mathrm{~mm} \mathrm{cpx}$ \\
\hline 3 & gabbros & Rajmi & & OM19-25 & No. 6 & 56.33172 & 24.62788 & 2 & s & & 5 aphyric & 190 & 67 & $\mathrm{P}$ & \\
\hline 3 & gabbros & Rajmi & & OM19-25 & No. 7 & 56.33172 & 24.62788 & 2 & s & & 6 aphyric & 180 & 72 & $\mathrm{P}$ & \\
\hline 3 & gabbros & Rajmi & & OM19-25 & No. 8 & 56.33172 & 24.62788 & 2 & s & & 0 dolerite & 208 & 42 & $\mathrm{P}$ & \\
\hline 3 & gabbros & Rajmi & & OM19-25 & No. 9 & 56.33172 & 24.62788 & 2 & s & & 7 porphyritic & 198 & 70 & $\mathrm{P}$ & \\
\hline 3 & gabbros & Rajmi & & OM19-25 & No. 10 & 56.33172 & 24.62788 & 2 & $\mathrm{~s}$ & & 8 aphyric & 190 & 87 & $\mathrm{P}$ & \\
\hline 3 & gabbros & Rajmi & & OM19-25 & No. 11 & 56.33172 & 24.62788 & 2 & s & & 2 aphyric & 170 & 88 & $\mathrm{P}$ & Sample \\
\hline 3 & gabbros & Rajmi & & OM19-26 & No. 1 & 56.33168 & 24.62819 & 2 & s & & $8 \mathrm{cpx}$ dolerite & 188 & 77 & $\mathrm{p}$ & \\
\hline 3 & gabbros & Rajmi & & OM19-26 & No. 2 & 56.33168 & 24.62819 & 1 & s & & $2 \mathrm{cpx}$ ol dolerite & 194 & 73 & $\mathrm{P}$ & \\
\hline 3 & gabbros & Rajmi & & OM19-26 & No. 3 & 56.33168 & 24.62819 & 2 & s & & 4 aphyric basalt & 180 & 82 & $\mathrm{P}$ & Sample \\
\hline 3 & gabbros & Rajmi & & OM19-26 & No. 4 & 56.33168 & 24.62819 & 2 & s & & 0 cpx basalt & 176 & 73 & $\mathrm{P}$ & \\
\hline 3 & gabbros & Rajmi & & OM19-26 & No. 4 & 56.33168 & 24.62819 & 2 & $\mathrm{~s}$ & & $0 \mathrm{cpx}$ basalt & 338 & 86 & $P$ & bended \\
\hline 3 & gabbros & Rajmi & & OM19-26 & No. 5 & 56.33168 & 24.62819 & 2 & $\mathrm{~s}$ & & $3 \mathrm{cpx}$ dolerite & 166 & 86 & $\mathrm{P}$ & \\
\hline 3 & gabbros & Rajmi & & OM19-26 & No. 5 & 56.33168 & 24.62819 & 2 & s & & $3 \mathrm{cpx}$ dolerite & 138 & 36 & $\mathrm{P}$ & sheared \\
\hline 3 & gabbros & Rajmi & & OM19-26 & No. 6 & 56.33168 & 24.62819 & 2 & $\mathrm{~s}$ & & 2 cpx pl dolerite & 160 & 81 & $\mathrm{P}$ & \\
\hline 3 & gabbros & Rajmi & & OM19-26 & No. 7 & 56.33168 & 24.62819 & 2 & $\mathrm{~s}$ & & $1 \mathrm{cpx}$ basalt & 214 & 90 & $\mathrm{P}$ & \\
\hline 3 & gabbros & Rajmi & & OM19-26 & No. 8 & 56.33168 & 24.62819 & 2 & $\mathrm{~s}$ & & $7 \mathrm{cpx}$ basalt & 206 & 88 & $\mathrm{P}$ & \\
\hline 3 & gabbros & Rajmi & & OM19-26 & No. 9 & 56.33168 & 24.62819 & 2 & $\mathrm{~s}$ & & $5 \mathrm{cpx}$ dolerite & 190 & 82 & $\mathrm{P}$ & Sample \\
\hline 3 & gabbros & Rajmi & & OM19-26 & No. 10 & 56.33168 & 24.62819 & 2 & s & & cpx basalt & 204 & 80 & $\mathrm{P}$ & \\
\hline 3 & $\mathrm{v}_{1}$ & Rajmi & & OM19-31 & No. 1 & 56.37509 & 24.62365 & 2 & s & & 3 aphyric basalt & 234 & 54 & $\mathrm{p}$ & massive flow host \\
\hline 3 & v1 & Rajmi & & OM19-31 & No. 2 & 56.37509 & 24.62365 & 2 & $\mathrm{~s}$ & & & 212 & 54 & $\mathrm{P}$ & \\
\hline 3 & v1 & Rajmi & & OM19-31 & No. 3 & 56.37509 & 24.62365 & 2 & $\mathrm{~s}$ & & aphyric basalt & 210 & 56 & $\mathrm{P}$ & \\
\hline 3 & $\mathrm{~V} 1_{1}$ & Rajmi & & OM19-31 & No. 4 & 56.37509 & 24.62365 & 2 & s & $500+$ & & 230 & 55 & $\mathrm{P}$ & massive flow host \\
\hline 3 & v1 & Rajmi & & OM19-31 & No. 5 & 56.37509 & 24.62365 & 2 & $\mathrm{~s}$ & & sparsely cpx phyric & 239 & 57 & $\mathrm{P}$ & \\
\hline 3 & V1 & Rajmi & & OM19-31 & No. 6 & 56.37509 & 24.62365 & 2 & s & & aphyric basalt & 231 & 52 & $P$ & tuff breccia; cpx phyric basalt host \\
\hline 3 & $\mathrm{~V} 1$ & Rajmi & & OM19-32 & No. 1 & 56.37437 & 24.62359 & 2 & s & & 3 dolerite & 238 & 60 & $\mathrm{p}$ & \\
\hline 3 & $\mathrm{v} 1$ & Rajmi & & OM19-32 & No. 2 & 56.37437 & 24.62359 & 1 & $\mathrm{~s}$ & & dolerite & 55 & 70 & $P$ & \\
\hline
\end{tabular}




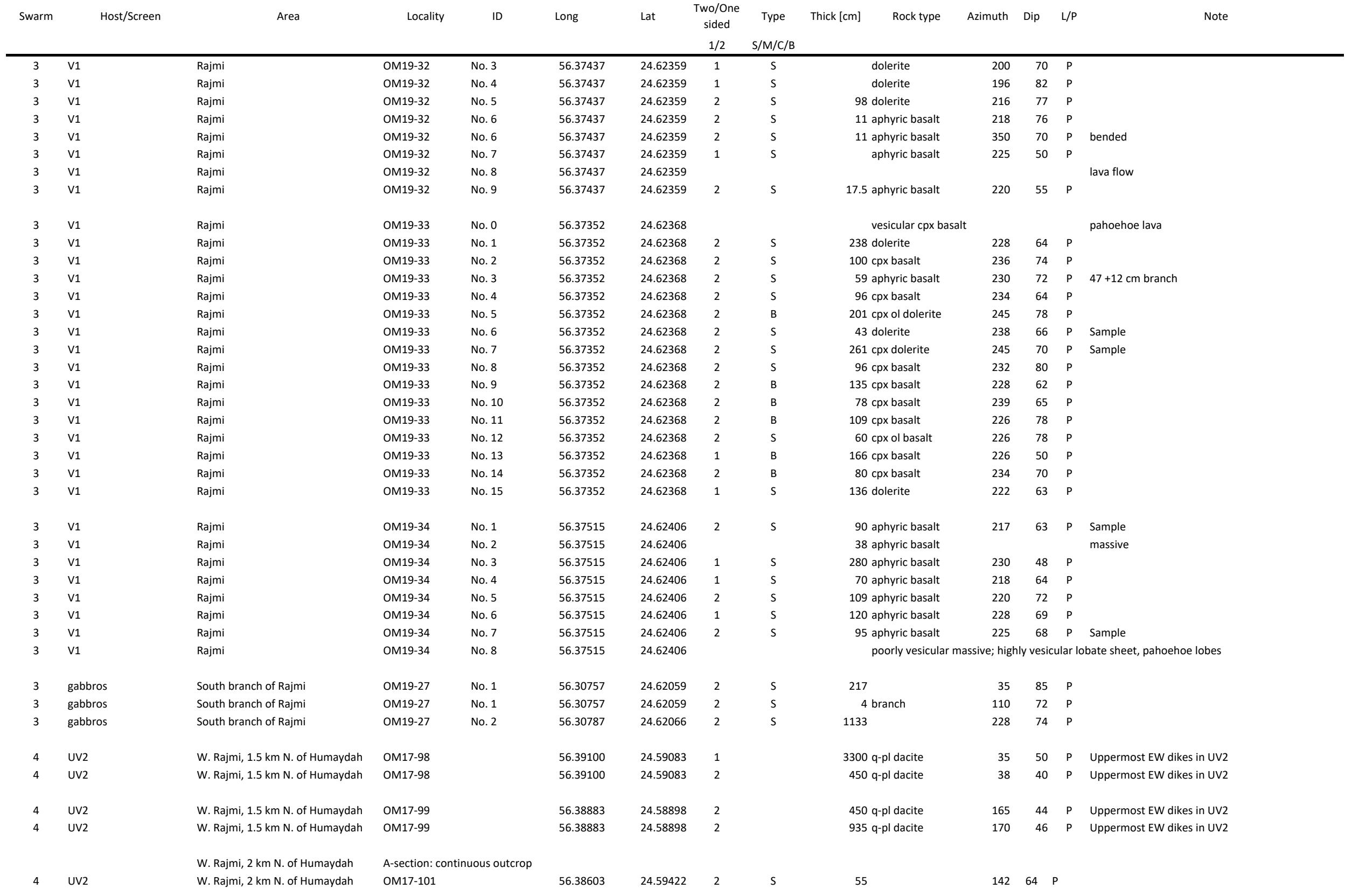




\begin{tabular}{|c|c|c|c|c|c|c|c|c|c|c|c|c|c|c|}
\hline \multirow[t]{2}{*}{ Swarm } & \multirow[t]{3}{*}{ Host/Screen } & \multirow[t]{2}{*}{ Area } & \multirow[t]{2}{*}{ Locality } & \multirow[t]{2}{*}{ ID } & \multirow[t]{2}{*}{ Long } & \multirow[t]{2}{*}{ Lat } & \multirow{2}{*}{$\begin{array}{c}\text { Two/One } \\
\text { sided } \\
1 / 2 \\
\end{array}$} & \multirow{2}{*}{$\begin{array}{c}\text { Type } \\
\text { S/M/C/B }\end{array}$} & \multirow[t]{2}{*}{ Thick [cm] } & \multirow[t]{2}{*}{ Rock type } & \multirow[t]{2}{*}{ Azimuth } & \multirow[t]{2}{*}{ Dip } & \multirow[t]{2}{*}{$L / P$} & \multirow[t]{2}{*}{ Note } \\
\hline & & & & & & & & & & & & & & \\
\hline 4 & & W. Rajmi, $2 \mathrm{~km} \mathrm{~N}$. of Humaydah & OM17-101 & & 56.38603 & 24.59422 & 2 & $\mathrm{~s}$ & & 0 dolerite & & & & \\
\hline 4 & UV2 & W. Rajmi, $2 \mathrm{~km} \mathrm{~N}$. of Humaydah & OM17-101 & & 56.38603 & 24.59422 & 0 & $\mathrm{~s}$ & & 15 dolerite & & & & \\
\hline 4 & UV2 & W. Rajmi, $2 \mathrm{~km} \mathrm{~N}$. of Humaydah & OM17-101 & & 56.38603 & 24.59422 & 2 & $\mathrm{~s}$ & & 8 c. aphyric boninite & 165 & $43 \quad \mathrm{P}$ & $\mathrm{P}$ & \\
\hline 4 & UV2 & W. Rajmi, $2 \mathrm{~km} \mathrm{~N}$. of Humaydah & OM17-101 & & 56.38603 & 24.59422 & 0 & $\mathrm{~s}$ & 80 & & & & & \\
\hline 4 & UV2 & W. Rajmi, $2 \mathrm{~km} \mathrm{~N}$. of Humaydah & OM17-101 & & 56.38603 & 24.59422 & 2 & $\mathrm{~s}$ & & 0 aphyric boninite & 150 & $60 \mathrm{P}$ & $\mathrm{P}$ & \\
\hline 4 & UV2 & W. Rajmi, $2 \mathrm{~km} \mathrm{~N}$. of Humaydah & OM17-101 & & 56.38603 & 24.59422 & 1 & $\mathrm{~s}$ & & 0 dolerite & 140 & $70 \mathrm{P}$ & $\mathrm{p}$ & \\
\hline 4 & UV2 & W. Rajmi, $2 \mathrm{~km} \mathrm{~N}$. of Humaydah & OM17-101 & & 56.38603 & 24.59422 & 0 & $\mathrm{~s}$ & & 0 dolerite & & & & \\
\hline \multirow[t]{2}{*}{4} & UV2 & W. Rajmi, $2 \mathrm{~km} \mathrm{~N}$. of Humaydah & OM17-101 & & 56.38603 & 24.59422 & 1 & $\mathrm{~s}$ & & 0 dolerite & 158 & $71 \mathrm{P}$ & $\mathrm{P}$ & \\
\hline & & W. Rajmi, $2 \mathrm{~km} \mathrm{~N}$. of Humaydah & B-section: con & inous outc & per stream of & & & & & & & & & \\
\hline 4 & UV2 & W. Rajmi, $2 \mathrm{~km} \mathrm{~N}$. of Humaydah & OM17-101 & & 56.38603 & 24.59422 & 1 & $\mathrm{~s}$ & & 5 aphyric boninite & 160 & $59 \mathrm{P}$ & $\mathrm{P}$ & \\
\hline 4 & UV2 & W. Rajmi, $2 \mathrm{~km} \mathrm{~N}$. of Humaydah & OM17-101 & & 56.38603 & 24.59422 & 2 & $\mathrm{~s}$ & & 3 aphyric boninite & 154 & $69 \mathrm{P}$ & $\mathrm{P}$ & \\
\hline 4 & uV2 & W. Rajmi, $2 \mathrm{~km} \mathrm{~N}$. of Humaydah & OM17-101 & & 56.38603 & 24.59422 & 0 & $\mathrm{~s}$ & & 9 dolerite & & & & \\
\hline 4 & UV2 & W. Rajmi, $2 \mathrm{~km} \mathrm{~N}$. of Humaydah & OM17-101 & & 56.38603 & 24.59422 & 2 & $\mathrm{~s}$ & & 8 aphyric boninite & 172 & $50 \mathrm{P}$ & $\mathrm{p}$ & \\
\hline 4 & UV2 & W. Rajmi, $2 \mathrm{~km} \mathrm{~N}$. of Humaydah & OM17-101 & & 56.38603 & 24.59422 & 2 & $\mathrm{~s}$ & & 2 aphyric boninite & 166 & $51 \mathrm{P}$ & $\mathrm{P}$ & Sample \\
\hline 4 & UV2 & W. Rajmi, $2 \mathrm{~km} \mathrm{~N}$. of Humaydah & OM17-101 & & 56.38603 & 24.59422 & & & & 3 ol-aug boninite pah & hoehoe & & & host \\
\hline 4 & UV2 & W. Rajmi, $2 \mathrm{~km} \mathrm{~N}$. of Humaydah & OM17-101 & & 56.38603 & 24.59422 & 2 & $\mathrm{~s}$ & & 2 aphyric boninite & 172 & $68 \mathrm{P}$ & $\mathrm{P}$ & Sample \\
\hline 4 & UV2 & W. Rajmi, $2 \mathrm{~km} \mathrm{~N}$. of Humaydah & OM17-101 & & 56.38603 & 24.59422 & 0 & $\mathrm{~s}$ & & 9 dolerite & & & & \\
\hline 4 & UV2 & W. Rajmi, $2 \mathrm{~km} \mathrm{~N}$. of Humaydah & OM17-101 & & 56.38603 & 24.59422 & 2 & $\mathrm{~s}$ & & $6 \mathrm{cpx}$ boninite & 164 & $77 P$ & $\mathrm{P}$ & \\
\hline 4 & UV2 & W. Rajmi, $2 \mathrm{~km} \mathrm{~N}$. of Humaydah & OM17-101 & & 56.38603 & 24.59422 & 0 & $\mathrm{~s}$ & & 4 dolerite & & & & \\
\hline 4 & UV2 & W. Rajmi, $2 \mathrm{~km} \mathrm{~N}$. of Humaydah & OM17-101 & & 56.38603 & 24.59422 & 2 & s & & 0 aphyric boninite & 174 & $72 \mathrm{P}$ & $P$ & \\
\hline 4 & uV2 & W. Rajmi, $2 \mathrm{~km} \mathrm{~N}$. of Humaydah & OM17-102 & & 56.38171 & 24.59512 & 2 & s & & 5 cpx boninite & 162 & $68 \mathrm{P}$ & $P$ & \\
\hline 4 & UV2 & W. Rajmi, $2 \mathrm{~km} \mathrm{~N}$. of Humaydah & OM17-102 & & 56.38171 & 24.59512 & 2 & $\mathrm{~s}$ & & 5 ol-aug boninite & 144 & $60 \mathrm{P}$ & $P$ & \\
\hline 4 & UV2 & W. Rajmi, $2 \mathrm{~km} \mathrm{~N}$. of Humaydah & OM17-102 & & 56.38171 & 24.59512 & 2 & c & & 1 aphyric boninite & 156 & $52 \mathrm{P}$ & $\mathrm{P}$ & $17 \mathrm{~cm}$ thick cpx phyric boninite in center \\
\hline 4 & UV2 & W. Rajmi, $2 \mathrm{~km} \mathrm{~N}$. of Humaydah & OM17-102 & & 56.38171 & 24.59512 & 2 & $\mathrm{~s}$ & & $8 \mathrm{cpx}$ boninite & 168 & $54 \mathrm{P}$ & $P$ & \\
\hline 4 & UV2 & W. Rajmi, $2 \mathrm{~km} \mathrm{~N}$. of Humaydah & OM17-102 & & 56.38171 & 24.59512 & 2 & c & & 5 aphyric boninite & 120 & $44 \mathrm{P}$ & $\mathrm{P}$ & $32 \mathrm{~cm} \mathrm{cpx} \mathrm{phyric} \mathrm{boninite} \mathrm{in} \mathrm{center}$ \\
\hline 4 & UV2 & W. Rajmi, $2 \mathrm{~km} \mathrm{~N}$. of Humaydah & OM17-102 & & 56.38171 & 24.59512 & 2 & $\mathrm{~s}$ & & 5 aphyric boninite & 174 & $66 \mathrm{P}$ & $\mathrm{P}$ & \\
\hline 4 & UV2 & W. Rajmi, $2 \mathrm{~km} \mathrm{~N}$. of Humaydah & OM17-102 & & 56.38171 & 24.59512 & 2 & $\mathrm{~s}$ & & 3 aphyric boninite & 152 & $30 \mathrm{P}$ & $\mathrm{P}$ & \\
\hline 4 & UV2 & W. Rajmi, $2 \mathrm{~km} \mathrm{~N}$. of Humaydah & OM17-102 & & 56.38171 & 24.59512 & 2 & $\mathrm{~s}$ & & 7 aphyric boninite & 168 & $50 \mathrm{P}$ & $P$ & \\
\hline 4 & UV2 & W. Rajmi, $2 \mathrm{~km} \mathrm{~N}$. of Humaydah & OM17-102 & & 56.38171 & 24.59512 & 2 & $\mathrm{~s}$ & & 5 aphyric boninite & 126 & $46 \mathrm{P}$ & $\mathrm{P}$ & westernside chilled margin elased upper \\
\hline 4 & UV2 & W. Rajmi, $2 \mathrm{~km} \mathrm{~N}$. of Humaydah & OM17-102 & & 56.38171 & 24.59512 & 2 & $\mathrm{~s}$ & & 0 aphyric boninite & 135 & $58 \mathrm{P}$ & $3 \mathrm{P}$ & \\
\hline 4 & UV2 & W. Rajmi, $2 \mathrm{~km} \mathrm{~N}$. of Humaydah & OM17-102 & & 56.38171 & 24.59512 & 2 & $\mathrm{~s}$ & & 0 aphyric boninite & 165 & $56 \mathrm{P}$ & $\mathrm{P}$ & become narrow on foot \\
\hline 4 & UV2 & W. Rajmi, $2 \mathrm{~km} \mathrm{~N}$. of Humaydah & OM17-102 & & 56.38171 & 24.59512 & 2 & $\mathrm{~s}$ & & 2 aphyric boninite & 158 & $52 \mathrm{P}$ & $P$ & \\
\hline 4 & UV2 & W. Rajmi, $2 \mathrm{~km} \mathrm{~N}$. of Humaydah & OM17-102 & & 56.38171 & 24.59512 & 2 & $\mathrm{~s}$ & & 5 sparse cpx boninit & 158 & $57 \mathrm{P}$ & $\mathrm{P}$ & \\
\hline 4 & UV2 & W. Rajmi, $2 \mathrm{~km} \mathrm{~N}$. of Humaydah & OM17-102 & & 56.38171 & 24.59512 & 2 & s & & 1 sparse cpx boninit & 168 & $60 \mathrm{P}$ & $\mathrm{P}$ & \\
\hline 4 & Late intrusives & South branch of Rajmi & OM19-28 & No. 1 & 56.31644 & 24.59546 & 2 & s & 66 & & 190 & 88 & $P$ & \\
\hline 4 & Late intrusives & South branch of Rajmi & OM19-28 & No. 2 & 56.31659 & 24.59544 & 1 & $\mathrm{~s}$ & & & 342 & 60 & $P$ & \\
\hline 4 & Late intrusives & South branch of Rajmi & OM19-28 & No. 3 & 56.31659 & 24.59544 & 2 & s & & 5 ol gabbronorite & 140 & 56 & $P$ & felsic tornalite assimilated \\
\hline 4 & Late intrusives & North branch of W. Zabin & OM19-29 & No. 1 & 56.31816 & 24.59030 & 1 & s & & gabbronorite & 22 & 82 & $P$ & \\
\hline 4 & Late intrusives & North branch of W. Zabin & OM19-29 & No. 1 & 56.31816 & 24.59030 & 1 & $\mathrm{~s}$ & & gabbronorite & 234 & 20 & $P$ & sill-like dimention \\
\hline 4 & Late intrusives & North branch of W. Zabin & OM19-29 & No. 2 & 56.31816 & 24.59030 & & & & $6 \mathrm{Hbl}$ gabbronorite & & & & screen \\
\hline 4 & Late intrusives & North branch of W. Zabin & OM19-29 & No. 3 & 56.31816 & 24.59030 & 1 & $\mathrm{~s}$ & & 2 dolerite & 34 & 84 & $P$ & \\
\hline 4 & Late intrusives & North branch of W. Zabin & OM19-29 & No. 4 & 56.31816 & 24.59030 & 2 & $\mathrm{~s}$ & & $85 \mathrm{~mm}$ cpx dolerite & 56 & 86 & 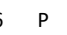 & \\
\hline 4 & Late intrusives & North branch of W. Zabin & OM19-29 & No. 5 & 56.31816 & 24.59030 & & & & gabbronorite & & & & screen; assimilation \\
\hline 4 & Late intrusives & North branch of W. Zabin & OM19-29 & No. 6 & 56.31816 & 24.59030 & 2 & $\mathrm{~s}$ & & 9 dolerite & 54 & 17 & $P$ & cut 5 and thrusted; Sample \\
\hline
\end{tabular}




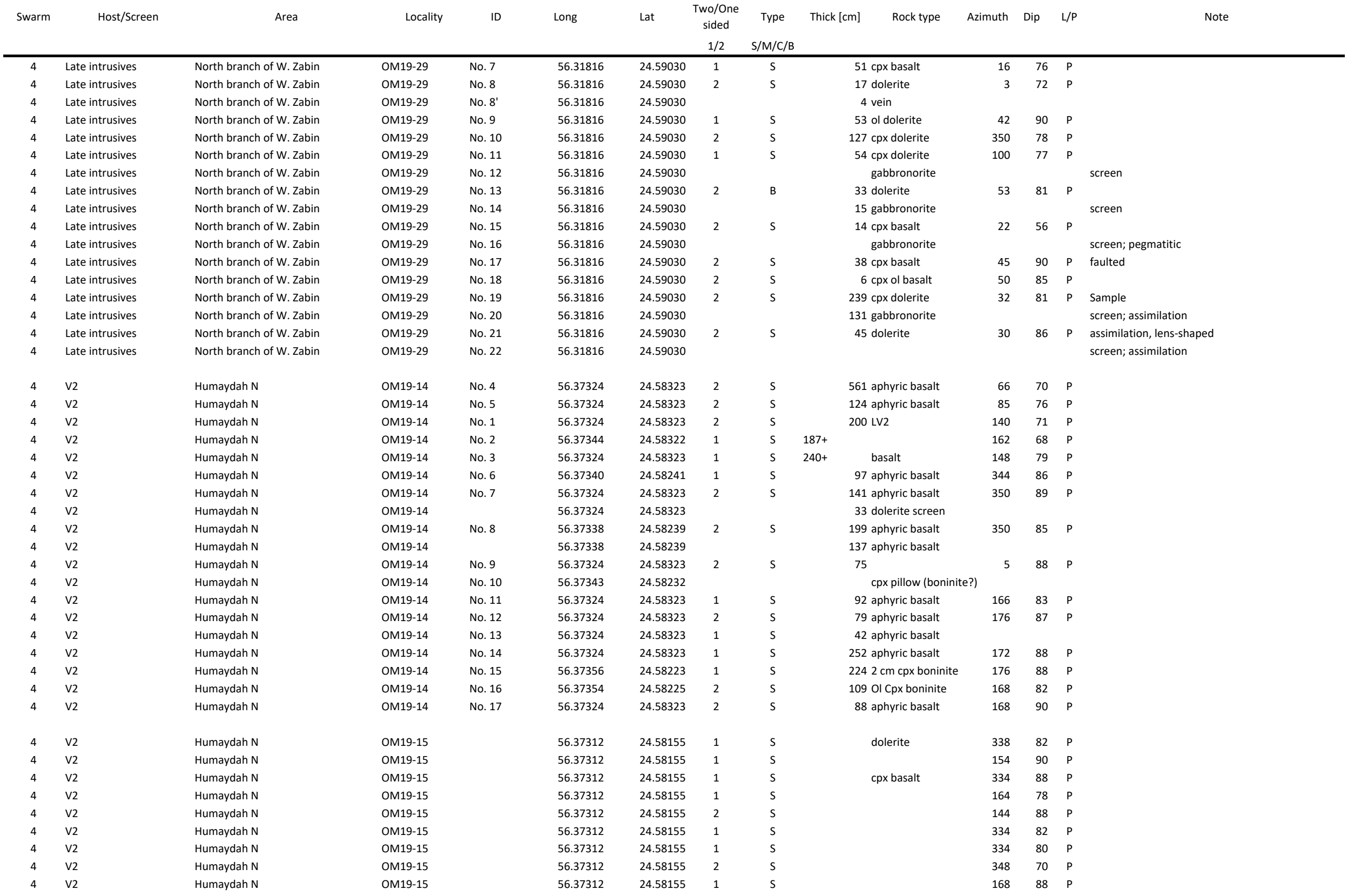




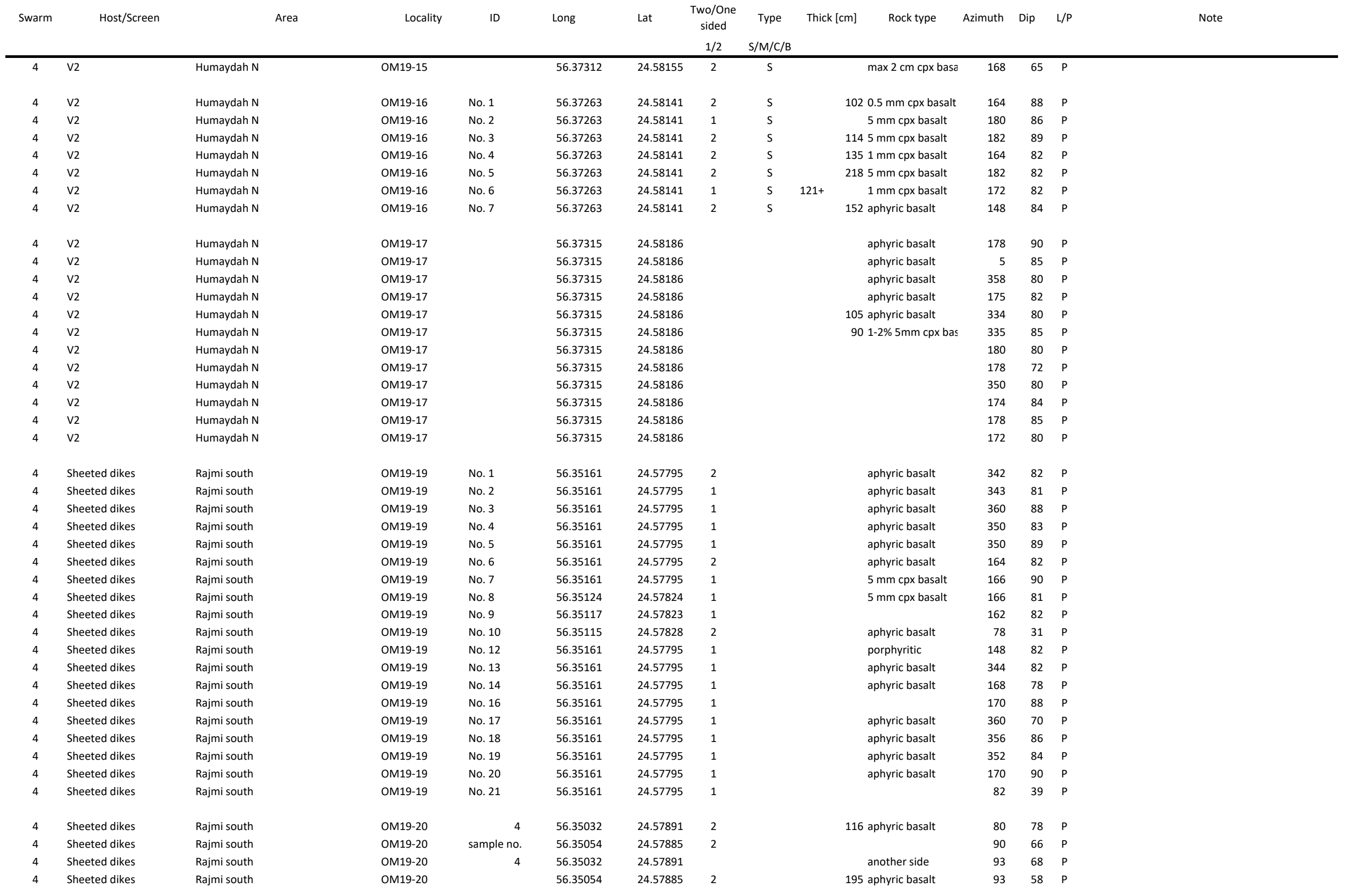




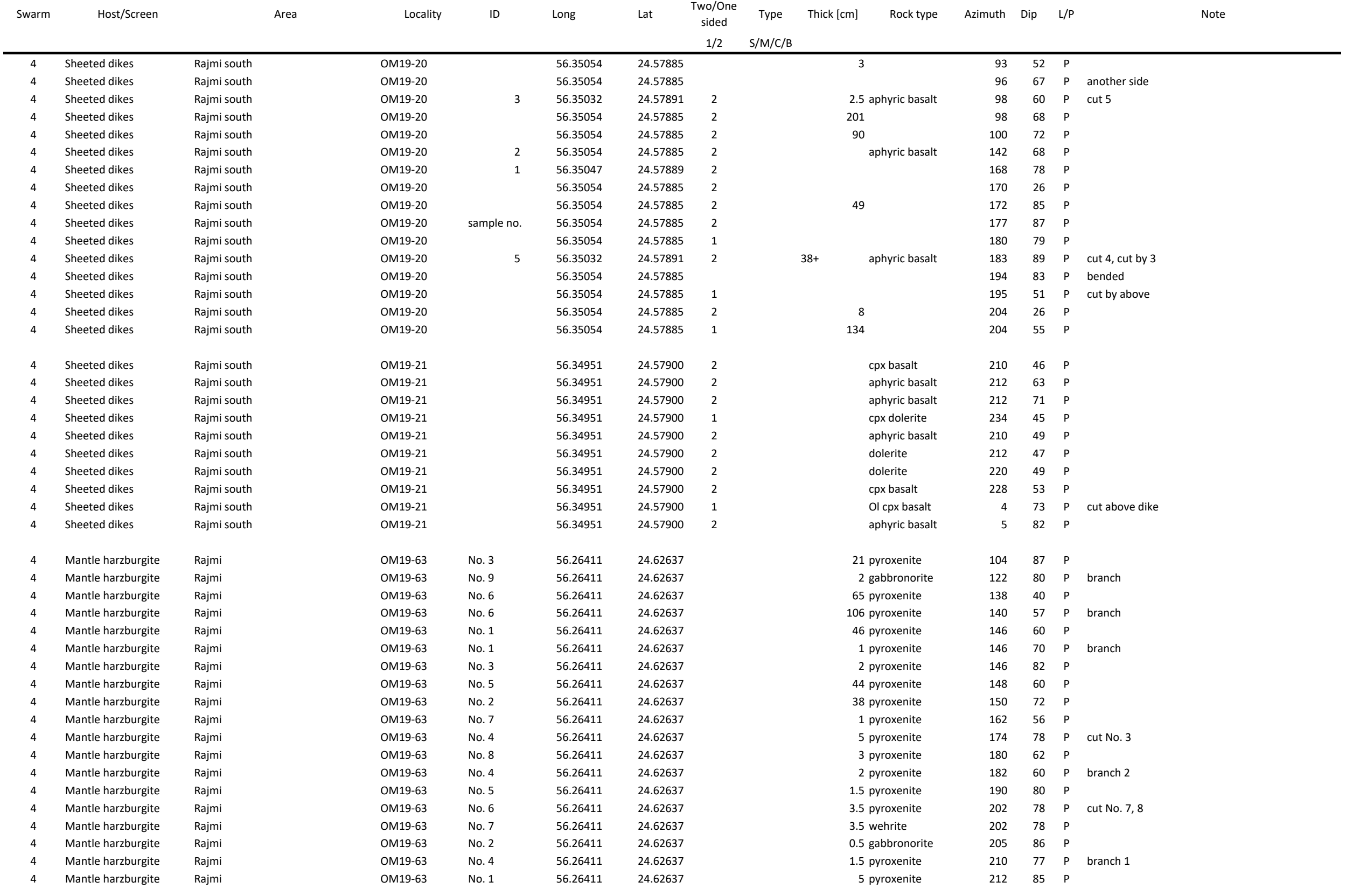




\begin{tabular}{|c|c|c|c|c|c|c|c|c|c|c|c|c|c|c|c|}
\hline \multirow[t]{2}{*}{ Swarm } & \multirow[t]{2}{*}{ Host/Screen } & \multirow{2}{*}{\multicolumn{2}{|c|}{ Area }} & \multirow[t]{2}{*}{ Locality } & \multirow[t]{2}{*}{ ID } & \multirow[t]{2}{*}{ Long } & \multirow[t]{2}{*}{ Lat } & \multirow{2}{*}{$\begin{array}{c}\text { Two/One } \\
\text { sided } \\
1 / 2 \\
\end{array}$} & \multirow{2}{*}{$\begin{array}{c}\text { Type } \\
\mathrm{S} / \mathrm{M} / \mathrm{C} / \mathrm{B}\end{array}$} & Thick [cm] & \multirow[t]{2}{*}{ Rock type } & \multirow[t]{2}{*}{ Azimuth } & \multirow[t]{2}{*}{ Dip } & \multirow[t]{2}{*}{$L / P$} & \multirow[t]{2}{*}{ Note } \\
\hline & & & & & & & & & & & & & & & \\
\hline 4 & Mantle harzburgite & Rajmi & & OM19-63 & No. 9 & 56.26411 & 24.62637 & & & & 2 gabbronorite & 214 & 90 & $P$ & \\
\hline 4 & Mantle harzburgite & Rajmi & & OM19-63 & No. 4 & 56.26411 & 24.62637 & & & & 2 pyroxenite & 222 & 88 & $\mathrm{P}$ & \\
\hline 4 & Mantle harzburgite & Rajmi & & OM19-63 & No. 8 & 56.26411 & 24.62637 & & & & 5 pyroxenite & 295 & 84 & $\mathrm{P}$ & \\
\hline 4 & Mantle harzburgite & Rajmi & & OM19-63 & No. 6 & 56.26411 & 24.62637 & & & & 6 pyroxenite & 300 & 80 & $\mathrm{P}$ & branch \\
\hline 4 & Late intrusives & Rajmi & & OM19-64 & & 56.26851 & 24.63120 & & & & gabbronorite & 156 & 66 & $\mathrm{P}$ & \\
\hline 4 & Late intrusives & Rajmi & & OM19-65 & No. 1 & 56.26917 & 24.63126 & & & & 5 felsic vein & 168 & 62 & $\mathrm{P}$ & \\
\hline 4 & Late intrusives & Rajmi & & OM19-65 & No. 1 & 56.26917 & 24.63126 & & & & 5 felsic vein & 224 & 90 & $\mathrm{P}$ & bended \\
\hline 4 & Late intrusives & Rajmi & & OM19-65 & No. 2 & 56.26917 & 24.63126 & & & & 2 felsic vein & 256 & 78 & $\mathrm{P}$ & \\
\hline 4 & Late intrusives & Rajmi & & OM19-65 & No. 3 & 56.26917 & 24.63126 & & & & 8 felsic vein & 229 & 80 & $\mathrm{P}$ & \\
\hline 4 & Late intrusives & Rajmi & & OM19-65 & No. 4 & 56.26917 & 24.63126 & & & & 8 felsic vein & 186 & 85 & $\mathrm{P}$ & \\
\hline 4 & Late intrusives & Rajmi & & OM19-65 & No. 5 & 56.26917 & 24.63126 & & & & 5 felsic vein & 25 & 72 & $\mathrm{P}$ & \\
\hline 4 & Late intrusives & Rajmi & & OM19-65 & No. 6 & 56.26917 & 24.63126 & & & $<9$ & & 352 & 87 & $\mathrm{P}$ & \\
\hline 4 & Late intrusives & Rajmi & & OM19-65 & No. 7 & 56.26917 & 24.63126 & & & $<2$ & felsic vein & 205 & 80 & $\mathrm{P}$ & \\
\hline 4 & Late intrusives & Rajmi & & OM19-65 & No. 7 & 56.26917 & 24.63126 & & & $<3$ & felsic vein & 28 & 67 & $\mathrm{P}$ & echelon \\
\hline 4 & Late intrusives & Rajmi & & OM19-65 & No. 1 & 56.26917 & 24.63126 & & & & & 240 & 78 & $\mathrm{P}$ & \\
\hline 4 & Late intrusives & Rajmi & & OM19-65 & No. 2 & 56.26917 & 24.63126 & & & & & 190 & 62 & $\mathrm{P}$ & \\
\hline 4 & Late intrusives & Rajmi & & OM19-65 & No. 3 & 56.26917 & 24.63126 & & & & & 214 & 66 & $\mathrm{P}$ & \\
\hline 4 & Late intrusives & Rajmi & & OM19-65 & No. 4 & 56.26917 & 24.63126 & & & 24 & 4 & 212 & 70 & $\mathrm{P}$ & \\
\hline 4 & Late intrusives & Rajmi & & OM19-65 & No. 5 & 56.26917 & 24.63126 & & & & 1 & 202 & 46 & $\mathrm{P}$ & \\
\hline 4 & Late intrusives & Rajmi & & OM19-65 & & 56.26917 & 24.63126 & & & & felsic vein & 162 & 62 & $\mathrm{P}$ & Host gabbronorite N4W75E \\
\hline 4 & Late intrusives & Rajmi & & OM19-65 & & 56.26917 & 24.63126 & & & & felsic vein & 144 & 71 & $\mathrm{P}$ & \\
\hline 4 & Late intrusives & Rajmi & & OM19-65 & & 56.26917 & 24.63126 & & & & felsic vein & 180 & 77 & $\mathrm{P}$ & Host gabbronorite N4E72E \\
\hline 4 & Late intrusives & Rajmi & & OM19-65 & & 56.26917 & 24.63126 & & & & felsic vein & 168 & 51 & $\mathrm{P}$ & \\
\hline 4 & Late intrusives & Rajmi & & OM19-66 & & 56.26760 & 24.63112 & & & & 5 hb diolite & 38 & 72 & $\mathrm{P}$ & sinistral \\
\hline 4 & Late intrusives & Rajmi & & OM19-66 & No. 1 & 56.26760 & 24.63112 & & & & 5 hb diolite & 39 & 60 & $\mathrm{P}$ & sinistral \\
\hline 4 & Late intrusives & Rajmi & & OM19-66 & & 56.26760 & 24.63112 & & & & 2 hb diolite & 48 & 64 & $P$ & Sample \\
\hline 4 & Late intrusives & Rajmi & & OM19-66 & No. 2 & 56.26760 & 24.63112 & & & & 5 & 212 & 66 & $P$ & dextral \\
\hline 4 & Late intrusives & Rajmi & & OM19-66 & No. 1 & 56.26760 & 24.63112 & & & & 5 hb diolite & 156 & 80 & $\mathrm{P}$ & \\
\hline 4 & Late intrusives & Rajmi & & ом19-66 & No. 1 & 56.26760 & 24.63112 & & & & & 318 & 46 & $P$ & \\
\hline 4 & Late intrusives & Rajmi & & OM19-67 & & 56.26629 & 24.63104 & & & & 5 hb gabbro & 72 & 62 & $\mathrm{P}$ & $3 \mathrm{~cm}$ thick $2 \mathrm{px}$ pyroxinite at chilled margin \\
\hline 4 & Late intrusives & Rajmi & & ом19-68 & No. 3 & 56.26600 & 24.63165 & & & & 1 hb diolite & 8 & 80 & $\mathrm{P}$ & dextral \\
\hline 4 & Late intrusives & Rajmi & & OM19-68 & No. 2 & 56.26600 & 24.63165 & & & & 5 hb gabbronorite & 35 & 80 & $\mathrm{P}$ & sinistral \\
\hline 4 & Late intrusives & Rajmi & & OM19-68 & & 56.26600 & 24.63165 & & & & hb diolite & 42 & 68 & $\mathrm{P}$ & \\
\hline 4 & Late intrusives & Rajmi & & OM19-68 & No. 2 & 56.26600 & 24.63165 & & & & 5 hb gabbronorite & 66 & 87 & $\mathrm{P}$ & \\
\hline 4 & Late intrusives & Rajmi & & OM19-68 & No. 2 & 56.26600 & 24.63165 & & & & 3 hb diolite & 212 & 80 & $\mathrm{P}$ & sinistral \\
\hline 4 & Late intrusives & Rajmi & & OM19-68 & & 56.26600 & 24.63165 & & & & hb diolite & 100 & 22 & $\mathrm{P}$ & \\
\hline 4 & Late intrusives & Rajmi & & OM19-68 & No. 3 & 56.26600 & 24.63165 & & & & 1 hb diolite & 140 & 53 & $\mathrm{P}$ & \\
\hline 4 & Late intrusives & Rajmi & & OM19-68 & No. 1 & 56.26600 & 24.63165 & & & 2 & 2 & 284 & 70 & $\mathrm{P}$ & sinistral \\
\hline 4 & Late intrusives & Rajmi & & OM19-68 & No. 1 & 56.26600 & 24.63165 & & & & 3 gabbronorite & 287 & 88 & $\mathrm{P}$ & dextral \\
\hline
\end{tabular}


$1 / 2 \quad \mathrm{~S} / \mathrm{M} / \mathrm{C} / \mathrm{B}$

$\begin{array}{ll}\text { Late intrusives } & \text { Rajmi } \\ \text { Late intrusives } & \text { Rajmi } \\ \text { Late intrusives } & \text { Rajmi } \\ \text { Late intrusives } & \text { Rajmi } \\ \text { Late intrusives } & \text { Rajmi } \\ \text { Late intrusives } & \text { Rajmi } \\ \text { Late intrusives } & \text { Rajmi } \\ & \\ \text { Late intrusives } & \text { Rajmi } \\ \text { Late intrusives } & \text { Rajmi } \\ \text { Late intrusives } & \text { Rajmi } \\ & \\ \text { Mantle harzburgite } & \text { Rajmi } \\ \text { Mantle harzburgite } & \text { Rajmi } \\ \text { Mantle harzburgite } & \text { Rajmi } \\ \text { Mantle harzburgite } & \text { Rajmi } \\ \text { Mantle harzburgite } & \text { Rajmi } \\ \text { Mantle harzburgite } & \text { Rajmi } \\ \text { Mantle harzburgite } & \text { Rajmi } \\ \text { Mantle harzburgite } & \text { Rajmi } \\ \text { Mantle harzburgite } & \text { Rajmi } \\ \text { Mantle harzburgite } & \text { Rajmi } \\ \text { Mantle harzburgite } & \text { Rajmi } \\ \text { Mantle harzburgite } & \text { Rajmi } \\ \text { Mantle harzburgite } & \text { Rajmi } \\ \text { Mantle harzburgite } & \text { Rajmi } \\ \text { Mantle harzburgite } & \text { Rajmi } \\ \text { Mantle harzburgite } & \text { Rajmi } \\ \text { Mantle harzburgite } & \text { Rajmi } \\ \text { Mantle harzburgite } & \text { Rajmi } \\ \text { Mantle harzburgite } & \text { Rajmi } \\ \text { Mantle harzburgite } & \text { Rajmi } \\ \text { Mantle harzburgite } & \text { Rajmi } \\ \text { Mantle harzburgite } & \text { Rajmi } \\ \text { Mantle harzburgite } & \text { Rajmi } \\ \text { Mantle harzburgite } & \text { Rajmi } \\ \text { Mantle harzburgite } & \text { Rajmi } \\ \text { Mantle harzburgite } & \text { Rajmi } \\ \text { Mantle harzburgite } & \text { Rajmi } \\ \text { Mantle harzburgite } & \text { Rajmi } \\ \text { Mantle harzburgite } & \text { Rajmi } \\ \text { Mantle harzburgite } & \text { Rajmi } \\ \text { Mantle harzburgite } & \text { Rajmi } \\ \text { Mantle harzburgite } & \text { Rajmi } \\ \text { Mantle harzburgite } & \text { Rajmi } \\ \text { Mantle harzburgite } & \text { Rajmi }\end{array}$

OM19-69

OM19-69

No. 2

\section{No. 2}

No. 1

No. 1

56.26635

56.26635

24.63253

OM19-69

OM19-69 No. 1

56.26635

56.26635

24.63253

24.63253

\begin{tabular}{ll}
56.26635 & 24.63253 \\
\hline
\end{tabular}

$56.26635 \quad 24.63253$

\begin{tabular}{llll} 
OM19-70 No. 1 & 56.26605 & 24.63292 \\
\hline
\end{tabular}

$\begin{array}{llll}\text { M19-70 No. } 2 & 56.26605 & 24.63292 \\ & & 56.2605 & 2.63292\end{array}$

No. 2

OM19-62 No. 4

OM19-62 No. 5

OM19-62 No. 6

OM19-62 No. 7

DM19-62 No. 8

$\begin{array}{ll}\text { OM19-62 } & \text { No. } 9 \\ \text { OM19-62 } & \text { No. } 10 \\ \text { OM1962 } & \text { No. }\end{array}$

OM19-62 No. 11

OM19-62 No. 12

OM19-62 No. 13

NM19-62 No.14

No. 1

OM19-62 No. 2

OM19-62 No. 4

OM19-62 No. 5

OM19-62 No. 6

OM19-62 No. 7

OM19-62 No. 8

OM19-62 No.10

OM19-62 No. 11

OM19-62 No. 12

OM19-62 No. 13

No.19-62

OM19-62 No. 2

OM19-62 No. 3

OM19-62 No. 5

OM19-62 No. 6

OM19-62
56.26605

24.63292

$\begin{array}{lll}56.25417 & 24.61878 \\ 56.25417 & 24.61878\end{array}$

$56.25417 \quad 24.61878$

$56.25417 \quad 24.61878$

$56.25417 \quad 24.61878$

$56.25417 \quad 24.61878$

$56.25417 \quad 24.61878$

$\begin{array}{ll}56.25417 & 24.61878 \\ 56.25417 & 24.61878\end{array}$

$56.25417 \quad 24.61878$

$56.25417 \quad 24.61878$

$56.25417 \quad 24.61878$

$56.25417 \quad 24.61878$

$56.25417 \quad 24.61878$

$56.25417 \quad 24.61878$

$56.25417 \quad 24.61878$

$56.25417 \quad 24.61878$

$56.25417 \quad 24.61878$

$\begin{array}{ll}56.25417 & 24.61878\end{array}$

$56.25417 \quad 24.61878$

$56.25417 \quad 24.61878$

$56.25417 \quad 24.61878$

$\begin{array}{ll}56.25417 & 24.61878 \\ 5625417 & 2461878\end{array}$

$56.25417 \quad 24.61878$

$\begin{array}{ll}56.25417 & 24.61878\end{array}$

$\begin{array}{ll}56.25417 & 24.61878\end{array}$

$56.25417 \quad 24.61878$

$56.25417 \quad 24.61878$

$56.25417 \quad 24.61878$

$56.25417 \quad 24.61878$

$56.25417 \quad 24.61878$

$\begin{array}{ll}56.25417 & 24.61878\end{array}$
No. 7
Thick [cm] Rock type Azimuth Dip L/P

ajmi

1

$\begin{array}{rrrl}18 & 87 & \mathrm{P} & \text { dextral } \\ 26 & 82 & \mathrm{P} & \text { sinistral } \\ 200 & 90 & \mathrm{P} & \text { sinistral } \\ 206 & 72 & \mathrm{P} & \text { sinistral } \\ 98 & 88 & \mathrm{P} & \\ 132 & 67 & \mathrm{P} & \text { dextral } \\ 154 & 80 & \mathrm{P} & \\ & & & \\ 4 & 87 & \mathrm{P} & \text { sinistral } \\ 172 & 80 & \mathrm{P} & \text { sinistral } \\ 38 & 84 & \mathrm{P} & \text { sinistral }\end{array}$

2

$38 \quad 84 \quad \mathrm{P}$ sinistral

\begin{tabular}{lllll}
7 & ol gabbronorite & 216 & 84 & $\mathrm{P}$ \\
\hline & 5 ol gabbronorite & 207 & 84 & $\mathrm{P}$
\end{tabular}

7.5 ol-2px pyroxinite $\quad 346 \quad 80 \quad P \quad$ Harzburgite foliation: N25E24E

8 ol gabbronorite $\quad 34 \quad 81 \quad \mathrm{P}$

32 px pyroxinite $\quad 172 \quad 80 \quad P$

$6.52 \mathrm{px}$ pyroxinite $\quad 155 \quad 70 \quad \mathrm{P}$

16 ol gabbronorite $\quad 203 \quad 80 \quad \mathrm{P}$

$0.62 \mathrm{px}$ pyroxinite $\quad 76 \quad 83 \quad \mathrm{p}$

$\begin{array}{lllll}1.52 \text { px pyroxinite } & 350 & 83 & \mathrm{P} & \text { cross with No. } 8\end{array}$

$\begin{array}{llll}208 & 83 & P & \text { Harzburgite foliation: N52W15N with weak liniation }\end{array}$

1.5 ol gabbronorite $\quad 202 \quad 80 \quad \mathrm{P}$

$\begin{array}{lllll}3 \text { ol gabbronorite } & 206 & 84 & \mathrm{P} & \text { divide to No. } 11\end{array}$

2 ol gabbronorite $\quad 214 \quad 80 \quad \mathrm{P}$

2 ol gabbronorite $\quad 202 \quad 80 \quad P \quad$ Harzburgite foliation: N44E14N, N45E22S, N57E22S

10 pyroxenite

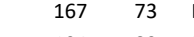

2 ol gabbronorite $\quad 188 \quad 82 \quad \mathrm{P}$

1 pyroxenite $\quad 180 \quad 84 \quad \mathrm{P}$

4 ol gabbronorite $\quad 246 \quad 80 \quad \mathrm{P}$

1.5 ol gabbronorite $\quad 206 \quad 88 \quad \mathrm{P}$

2.5 pyroxenite $\quad 188 \quad 73 \quad P$

19 ol gabbronorite $\quad 180 \quad 85 \quad \mathrm{P}$

3 pyroxenite $\quad 202 \quad 79 \quad \mathrm{P}$

6 pyroxenite $\quad 210 \quad 74 \quad \mathrm{P}$

6 pyroxenite $\quad 60-60$ P

$\begin{array}{lrlll}6.5 & \text { ol gabbronorite } & 200 & 77 & P\end{array}$

$\begin{array}{llll}4.5 \text { ol gabbronorite } & 200 & 77 & \mathrm{P} \\ 1.5 \text { pyroxenite } & 172 & 81 & \mathrm{P}\end{array}$

21 gabbronorite $\quad 222 \quad 54 \quad P$

2.5 gabbronorite $\quad 210 \quad 82 \quad \mathrm{P}$

$\begin{array}{llll}1 \text { pyroxenite } & 174 & 62 & \mathrm{P} \\ 3 \text { gabbronorite } & 210 & 76 & \mathrm{P}\end{array}$

5 pyroxenite $\quad 217 \quad 86 \quad P$

1 gabbronorite $\quad 215 \quad 89 \quad \mathrm{P}$ 


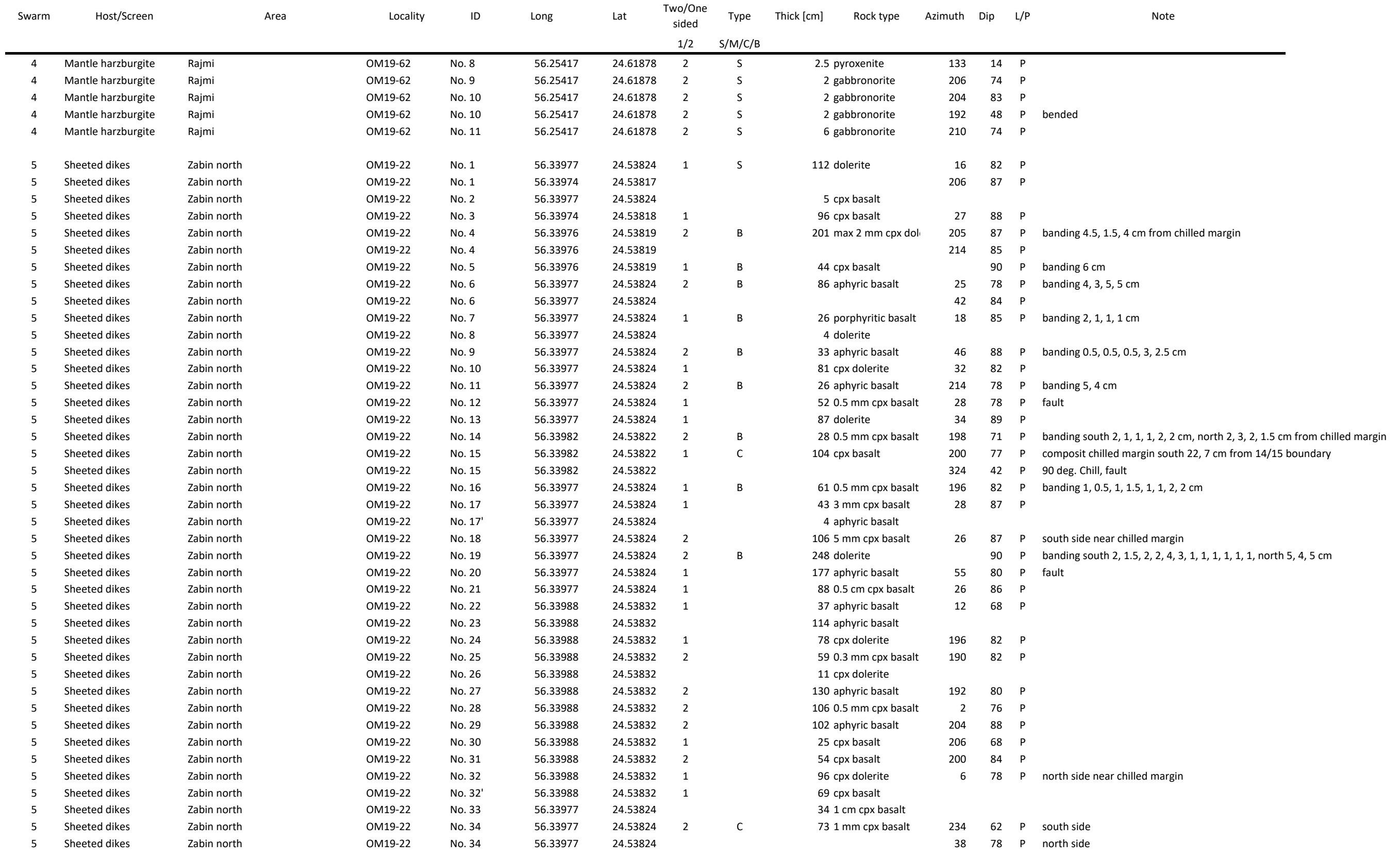




\begin{tabular}{|c|c|c|c|c|c|c|c|c|c|c|c|c|c|c|}
\hline \multirow[t]{2}{*}{ Swarm } & \multirow[t]{2}{*}{ Host/Screen } & \multirow[t]{2}{*}{ Area } & \multirow[t]{2}{*}{ Locality } & \multirow[t]{2}{*}{ ID } & \multirow[t]{2}{*}{ Long } & \multirow[t]{2}{*}{ Lat } & \multirow{2}{*}{$\begin{array}{c}\text { Two/One } \\
\text { sided } \\
1 / 2\end{array}$} & \multirow{2}{*}{$\begin{array}{c}\text { Type } \\
S / M / C / B\end{array}$} & \multirow[t]{2}{*}{ Thick [cm] } & \multirow[t]{2}{*}{ Rock type } & \multirow[t]{2}{*}{ Azimuth } & \multirow[t]{2}{*}{ Dip } & \multirow[t]{2}{*}{$L / P$} & \multirow[t]{2}{*}{ Note } \\
\hline & & & & & & & & & & & & & & \\
\hline 5 & Sheeted dikes & Zabin north & OM19-22 & No. 35 & 56.33977 & 24.53824 & 2 & & & $55 \mathrm{~mm}$ cpx basalt & 202 & 80 & $P$ & intruding 34 \\
\hline 5 & Sheeted dikes & Zabin north & OM19-22 & No. 36 & 56.33977 & 24.53824 & & & & $980.2 \mathrm{~mm}$ cpx basalt & & & & \\
\hline 5 & Sheeted dikes & Zabin north & OM19-22 & No. 37 & 56.33977 & 24.53824 & 2 & & & $311 \mathrm{~mm} \mathrm{cpx}$ basalt & 27 & 88 & $\mathrm{P}$ & \\
\hline 5 & Sheeted dikes & Zabin north & OM19-22 & No. 38 & 56.33977 & 24.53824 & & & & $77 \mathrm{cpx}$ basalt & & & & \\
\hline 5 & Sheeted dikes & Zabin north & OM19-22 & No. 39 & 56.33977 & 24.53824 & 2 & B & & $371 \mathrm{~mm}$ cpx basalt & 180 & 76 & $\mathrm{P}$ & banding $3,2,4 \mathrm{~cm}$ \\
\hline 5 & Sheeted dikes & Zabin north & OM19-22 & No. 40 & 56.33977 & 24.53824 & 1 & & & $621 \mathrm{~mm}$ cpx basalt & 186 & 80 & $\mathrm{P}$ & \\
\hline 5 & Sheeted dikes & Zabin north & OM19-22 & No. 41 & 56.33977 & 24.53824 & 1 & B & & $581 \mathrm{~mm}$ cpx basalt & 200 & 87 & $\mathrm{P}$ & banding $1.5,4 \mathrm{~cm}$ \\
\hline 5 & Sheeted dikes & Zabin north & OM19-22 & No. 42 & 56.33977 & 24.53824 & 1 & & & $832 \mathrm{~mm}$ cpx basalt & 200 & 87 & $\mathrm{P}$ & intruded by 44,43 \\
\hline 5 & Sheeted dikes & Zabin north & OM19-22 & No. 44 & 56.33977 & 24.53824 & 2 & & & $245 \mathrm{~mm}$ cpx basalt & 27 & 55 & $\mathrm{P}$ & bended south \\
\hline 5 & Sheeted dikes & Zabin north & OM19-22 & No. 43 & 56.33977 & 24.53824 & 2 & & & $121 \mathrm{~mm}$ cpx basalt & 200 & 82 & $\mathrm{P}$ & \\
\hline 5 & Sheeted dikes & Zabin north & OM19-22 & No. 43 & 56.33977 & 24.53824 & & & & $221 \mathrm{~mm}$ cpx basalt & 180 & 77 & $\mathrm{p}$ & branch; intruding to 42,44 \\
\hline 5 & Sheeted dikes & Zabin north & OM19-22 & No. 45 & 56.33977 & 24.53824 & 1 & & & 21 vesicular $1 \mathrm{~mm} \mathrm{cpx}$ & 12 & 84 & $\mathrm{P}$ & \\
\hline 5 & Sheeted dikes & Zabin north & OM19-22 & No. 46 & 56.33977 & 24.53824 & 2 & B & & $501 \mathrm{~mm}$ cpx basalt & 25 & 82 & $\mathrm{p}$ & banding $3,1,3 \mathrm{~cm}$ \\
\hline 5 & Sheeted dikes & Zabin north & OM19-22 & No. 47 & 56.33977 & 24.53824 & 1 & & & $611 \mathrm{~mm}$ cpx basalt & 225 & 86 & $\mathrm{p}$ & include $24 \mathrm{~cm}$ wide patchy tuff breccia \\
\hline 5 & Sheeted dikes & Zabin north & OM19-22 & No. 48 & 56.33977 & 24.53824 & 1 & & & $730.5 \mathrm{~mm} \mathrm{cpx} \mathrm{basalt}$ & 23 & 89 & $\mathrm{P}$ & \\
\hline 5 & Sheeted dikes & Zabin north & OM19-22 & No. 49 & 56.34002 & 24.53839 & & & & 91 tuff breccia, glassy & & & & agglutinate \\
\hline 5 & Sheeted dikes & Zabin north & OM19-22 & No. 51 & 56.33977 & 24.53824 & 2 & & & $76 \mathrm{cpx}$ dolerite & 231 & 86 & $\mathrm{P}$ & feeder dike of 49 ; intruded by 50 \\
\hline 5 & Sheeted dikes & Zabin north & OM19-22 & No. 52 & 56.33977 & 24.53824 & 2 & & & $11 \mathrm{max} 5 \mathrm{~mm}$ cpx dol. & 34 & 82 & $\mathrm{P}$ & \\
\hline 5 & Sheeted dikes & Zabin north & OM19-22 & No. 52 & 56.33977 & 24.53824 & & & & & 50 & 90 & $\mathrm{P}$ & north side \\
\hline 5 & Sheeted dikes & Zabin north & OM19-22 & No. 53 & 56.34001 & 24.53843 & 1 & & & $981 \mathrm{~mm}$ cpx basalt & 48 & 86 & $\mathrm{P}$ & \\
\hline 5 & Sheeted dikes & Zabin north & OM19-22 & No. 54 & 56.33977 & 24.53824 & 2 & & & $0.2 \mathrm{~mm}$ cpx basalt & 159 & 73 & $\mathrm{P}$ & bended north \\
\hline 5 & Sheeted dikes & Zabin north & OM19-22 & No. 55 & 56.33977 & 24.53824 & 2 & & & $485 \mathrm{~mm}$ cpx basalt & 218 & 72 & $\mathrm{P}$ & \\
\hline 5 & Sheeted dikes & Zabin north & OM19-22 & No. 56 & 56.33977 & 24.53824 & 2 & & & 59 aphyric basalt & 102 & 45 & $\mathrm{P}$ & \\
\hline 5 & Sheeted dikes & Zabin north & OM19-22 & No. 56 & 56.33977 & 24.53824 & & & & 31 & 226 & 67 & $\mathrm{P}$ & bended north \\
\hline 5 & Sheeted dikes & Zabin north & OM19-22 & No. 57 & 56.33977 & 24.53824 & & & & $.645 \mathrm{~mm}$ cpx basalt & & & & screen \\
\hline 5 & Sheeted dikes & Zabin north & OM19-22 & No. 58 & 56.33977 & 24.53824 & 2 & B & & 89 dolerite & 30 & 87 & $\mathrm{P}$ & banding $15 \mathrm{~cm}$ \\
\hline 5 & Sheeted dikes & Zabin north & OM19-22 & No. 59 & 56.33977 & 24.53824 & 2 & B & & $225 \mathrm{~mm}$ cpx basalt & 211 & 81 & $\mathrm{P}$ & banding $10,6,10 \mathrm{~cm}$; intruded by 60 and 61 \\
\hline 5 & Sheeted dikes & Zabin north & OM19-22 & No. 60 & 56.33977 & 24.53824 & 2 & & & 37 aphyric basalt & 33 & 81 & $\mathrm{P}$ & \\
\hline 5 & Sheeted dikes & Zabin north & OM19-22 & No. 61 & 56.33977 & 24.53824 & & & & 1 & & & & \\
\hline 5 & Sheeted dikes & Zabin north & OM19-22 & No. $60^{\prime}$ & 56.33977 & 24.53824 & & & & $61 \mathrm{cpx}$ dolerite & & & & screen \\
\hline 5 & Sheeted dikes & Zabin north & OM19-22 & No. 61' & 56.33977 & 24.53824 & 2 & & & 25 aphyric basalt & 250 & 74 & $\mathrm{P}$ & fault \\
\hline 5 & Sheeted dikes & Zabin north & OM19-22 & No. 62 & 56.33977 & 24.53824 & & & & $143 \mathrm{~mm}$ cpx basalt & & & & screen \\
\hline 5 & Sheeted dikes & Zabin north & OM19-22 & No. 63 & 56.33977 & 24.53824 & 1 & & & 16 aphyric basalt & 233 & 77 & $\mathrm{P}$ & \\
\hline 5 & Sheeted dikes & Zabin north & OM19-22 & No. 64 & 56.33977 & 24.53824 & 2 & & & 26 aphyric basalt & 216 & 85 & $\mathrm{P}$ & \\
\hline 5 & Sheeted dikes & Zabin north & OM19-22 & No. 65 & 56.33977 & 24.53824 & 1 & & & $791 \mathrm{~mm}$ cpx dolerite & 232 & 82 & $\mathrm{P}$ & \\
\hline 5 & Sheeted dikes & Zabin north & OM19-22 & No. 66 & 56.33977 & 24.53824 & 1 & & & 38 dolerite & 216 & 79 & $\mathrm{P}$ & \\
\hline 5 & Sheeted dikes & Zabin north & OM19-22 & No. 67 & 56.33977 & 24.53824 & 2 & & & 30 vesicular aphyric $b$ & 235 & 80 & $\mathrm{P}$ & \\
\hline 5 & Sheeted dikes & Zabin north & OM19-22 & No. 68 & 56.33977 & 24.53824 & & & & 02 tuff breccia, glassy & & & & agglutinate \\
\hline 5 & Sheeted dikes & Zabin north & OM19-22 & No. 69 & 56.33977 & 24.53824 & 2 & & & 6 aphyric basalt & 220 & 60 & $\mathrm{P}$ & wavy intruding 68 \\
\hline 5 & Sheeted dikes & Zabin north & OM19-22 & No. 70 & 56.33977 & 24.53824 & 2 & & & $261 \mathrm{~mm} \mathrm{cpx}$ basalt & 240 & 77 & $\mathrm{P}$ & $\max 2.5 \mathrm{~cm} \mathrm{cpx}$ \\
\hline 5 & Sheeted dikes & Zabin north & OM19-22 & No. 71 & 56.33977 & 24.53824 & 1 & & & $555 \mathrm{~mm}$ cpx basalt & 198 & 90 & $\mathrm{P}$ & wavy boundary with agglutinate \\
\hline 5 & Sheeted dikes & Zabin north & OM19-22 & No. $72^{\prime}$ & 56.33977 & 24.53824 & & & $132+$ & tuff breccia, glassy & & & & agglutinate \\
\hline 5 & Sheeted dikes & Zabin north & OM19-22 & No. 72 & 56.33977 & 24.53824 & 2 & B & & $80 \mathrm{cpx} \mathrm{pl} \mathrm{basalt}$ & 196 & 90 & $\mathrm{P}$ & intruding tuff breccia; banding $8 \mathrm{~cm}$ \\
\hline 5 & Sheeted dikes & Zabin north & OM19-22 & south No. 1 & 56.33977 & 24.53824 & 2 & & & & 27 & 85 & $\mathrm{P}$ & \\
\hline 5 & Sheeted dikes & Zabin north & OM19-22 & & 56.33977 & 24.53824 & 2 & & & & 27 & 79 & $\mathrm{P}$ & \\
\hline 5 & Sheeted dikes & Zabin north & OM19-22 & & 56.33977 & 24.53824 & 1 & & & & 28 & 71 & P & \\
\hline
\end{tabular}




\begin{tabular}{|c|c|c|c|c|c|c|c|c|c|c|c|c|c|}
\hline Swarm & Host/Screen & Area & Locality & ID & Long & Lat & $\begin{array}{c}\text { Two/One } \\
\text { sided } \\
1 / 2\end{array}$ & $\begin{array}{c}\text { Type } \\
\text { S/M/C/B }\end{array}$ & Thick [cm] & Azimuth & Dip & $L / P$ & Note \\
\hline 5 & Sheeted dikes & Zab' in & OM19-39 & & 56.32520 & 24.52831 & $\frac{1 / 2}{2}$ & $\mathrm{~s}$ & 8 aphyric basalt & 2 & 75 & $P$ & \\
\hline 5 & Sheeted dikes & Zab' in & OM19-39 & & 56.32520 & 24.52831 & 0 & $\mathrm{~s}$ & 83 aphyric dolerite & & & & \\
\hline 5 & Sheeted dikes & Zab' in & OM19-39 & & 56.32520 & 24.52831 & 2 & $\mathrm{~s}$ & 17 aphyric basalt & 12 & 83 & $\mathrm{P}$ & \\
\hline 5 & Sheeted dikes & Zab' in & OM19-39 & & 56.32520 & 24.52831 & 2 & $\mathrm{~s}$ & 19 aphyric dolerite & & & & \\
\hline 5 & Sheeted dikes & $Z a b^{\prime}$ in & OM19-39 & & 56.32520 & 24.52831 & 2 & $\mathrm{~s}$ & 49 & 12 & 83 & $\mathrm{P}$ & \\
\hline 5 & Sheeted dikes & $Z a b^{\prime}$ in & OM19-39 & & 56.32520 & 24.52831 & 0 & $\mathrm{~s}$ & 28 aphyric dolerite & & & & \\
\hline 5 & Sheeted dikes & Zab' in & OM19-39 & & 56.32520 & 24.52831 & 2 & $\mathrm{~s}$ & 40 aphyric basalt & 189 & 86 & $\mathrm{P}$ & \\
\hline 5 & Sheeted dikes & $Z a b^{\prime}$ in & OM19-39 & & 56.32520 & 24.52831 & 0 & $\mathrm{~s}$ & 55 aphyric dolerite & & & & \\
\hline 5 & Sheeted dikes & Zab' in & OM19-39 & & 56.32520 & 24.52831 & 2 & $\mathrm{~s}$ & 1 aphyric basalt & & & & \\
\hline 5 & Sheeted dikes & Zab' in & OM19-39 & & 56.32520 & 24.52831 & 0 & $\mathrm{~s}$ & 100 aphyric dolerite & & & & \\
\hline 5 & Sheeted dikes & Zab' in & OM19-39 & & 56.32520 & 24.52831 & 2 & $\mathrm{~s}$ & 63 aphyric basalt & 10 & 83 & $\mathrm{P}$ & Changes direction below \\
\hline 5 & Sheeted dikes & Zab' in & OM19-39 & & 56.32520 & 24.52831 & 2 & $\mathrm{~s}$ & 63 aphyric basalt & 288 & 85 & $\mathrm{P}$ & Changes direction above \\
\hline 5 & Sheeted dikes & $Z a b^{\prime}$ in & OM19-39 & & 56.32520 & 24.52831 & 0 & $\mathrm{~s}$ & 66 aphyric dolerite & & & & \\
\hline 5 & Sheeted dikes & Zab' in & OM19-39 & & 56.32520 & 24.52831 & 2 & $\mathrm{~s}$ & 4.6 aphyric basalt & 12 & 90 & $\mathrm{P}$ & \\
\hline 5 & Sheeted dikes & $Z a b^{\prime}$ in & OM19-39 & & 56.32520 & 24.52831 & 0 & $\mathrm{~s}$ & 40 aphyric dolerite & 174 & 88 & $\mathrm{P}$ & \\
\hline 5 & Sheeted dikes & Zab' in & OM19-39 & & 56.32520 & 24.52831 & 2 & $\mathrm{~s}$ & cpx boninite? & 2 & 84 & $\mathrm{P}$ & \\
\hline 5 & Sheeted dikes & $Z a b^{\prime}$ in & OM19-39 & & 56.32520 & 24.52831 & 2 & $\mathrm{~s}$ & $\mathrm{cpx}$ boninite? & 102 & 58 & $\mathrm{P}$ & \\
\hline 5 & Sheeted dikes & Zab' in & OM19-39 & No. 1 & 56.32520 & 24.52831 & 1 & $\mathrm{~s}$ & $46 \mathrm{cpx}$ basalt & 196 & 85 & $\mathrm{P}$ & \\
\hline 5 & Sheeted dikes & Zab' in & OM19-39 & No. 2 & 56.32520 & 24.52831 & 2 & $\mathrm{~s}$ & $31 \mathrm{cpx}$ basalt & 192 & 80 & $\mathrm{P}$ & \\
\hline 5 & Sheeted dikes & $Z a b^{\prime}$ in & OM19-39 & No. 3 & 56.32520 & 24.52831 & 1 & $\mathrm{~s}$ & aphyric basalt & 244 & 58 & $\mathrm{P}$ & \\
\hline 5 & Sheeted dikes & $Z a b^{\prime}$ in & OM19-39 & No. 4 & 56.32520 & 24.52831 & 1 & $\mathrm{~s}$ & aphyric basalt & 216 & 56 & $\mathrm{P}$ & \\
\hline 5 & Sheeted dikes & Zab' in & OM19-39 & No. 5 & 56.32520 & 24.52831 & 2 & $\mathrm{~s}$ & 106 aphyric basalt & 198 & 72 & $\mathrm{P}$ & \\
\hline 5 & Sheeted dikes & $Z a b^{\prime}$ in & OM19-39 & No. 6 & 56.32520 & 24.52831 & 2 & $\mathrm{~s}$ & & 0 & 82 & $\mathrm{P}$ & \\
\hline 5 & Sheeted dikes & Zab' in & OM19-39 & No. 0 & 56.32520 & 24.52831 & 1 & & aphyric basalt & 334 & 80 & $\mathrm{P}$ & \\
\hline 5 & Sheeted dikes & Zab' in & OM19-39 & No. 1 & 56.32520 & 24.52831 & & & aphyric basalt & 354 & 78 & $\mathrm{P}$ & \\
\hline 5 & Sheeted dikes & Zab' in & OM19-39 & No. 3 & 56.32520 & 24.52831 & & & aphyric basalt & 354 & 84 & $\mathrm{P}$ & \\
\hline 5 & Sheeted dikes & $Z a b^{\prime}$ in & OM19-39 & No. 4 & 56.32520 & 24.52831 & 2 & & aphyric basalt & 358 & 86 & $\mathrm{P}$ & \\
\hline 5 & Sheeted dikes & $Z a b^{\prime}$ in & OM19-39 & No. 5 & 56.32520 & 24.52831 & 1 & & aphyric basalt & 305 & 67 & $\mathrm{P}$ & \\
\hline 5 & Sheeted dikes & $Z a b^{\prime}$ in & OM19-39 & No. 7 & 56.32520 & 24.52831 & & & aphyric dolerite & 342 & 80 & $\mathrm{P}$ & \\
\hline 5 & Sheeted dikes & Zab' in & OM19-39 & No. 9 & 56.32520 & 24.52831 & & & & 184 & 86 & $\mathrm{P}$ & \\
\hline 5 & Gabbro & $Z a b^{\prime}$ in & OM19-41 & & 56.31627 & 24.53267 & & & $192 \mathrm{cpx}$ ol basalt & 196 & 84 & $\mathrm{P}$ & host: ol gabbro \\
\hline 5 & Gabbro & Zab' in & OM19-41 & & 56.31627 & 24.53267 & & & $114 \mathrm{cpx}$ basalt & 192 & 85 & $\mathrm{P}$ & host: ol gabbro \\
\hline 5 & Gabbro & Zab' in & OM19-41 & & 56.31627 & 24.53267 & & & $42 \mathrm{cpx}$ basalt & 186 & 62 & $\mathrm{P}$ & host: ol gabbro \\
\hline 5 & Gabbro & Zab' in & OM19-41 & & 56.31627 & 24.53267 & & & $88 \mathrm{cpx}$ basalt & 0 & 90 & $\mathrm{P}$ & host: ol gabbro \\
\hline 5 & Gabbro & Zab' in & OM19-41 & & 56.31627 & 24.53267 & & & $6 \mathrm{cpx}$ basalt & 186 & 84 & $\mathrm{P}$ & host: ol gabbro \\
\hline 5 & Gabbro & Zab' in & OM19-41 & & 56.31627 & 24.53267 & & & $42 \mathrm{cpx}$ basalt & 198 & 84 & $\mathrm{P}$ & host: ol gabbro \\
\hline 5 & Gabbro & Zab' in & OM19-41 & & 56.31627 & 24.53267 & & & $59 \mathrm{cpx}$ basalt & 6 & 88 & $\mathrm{P}$ & host: ol gabbro \\
\hline 5 & Sheeted dikes & $Z a b^{\prime}$ in & OM19-40 & No. 50 & 56.31921 & 24.53142 & 1 & $\mathrm{~s}$ & $38 \mathrm{cpx}$ basalt & 90 & 70 & $\mathrm{P}$ & \\
\hline 5 & Sheeted dikes & Zab' in & OM19-40 & No. 70 & 56.31921 & 24.53142 & 2 & $\mathrm{~s}$ & $10 \mathrm{cpx}$ ol basalt & 122 & 59 & $\mathrm{P}$ & echelon \\
\hline 5 & Sheeted dikes & Zab' in & OM19-40 & No. $3^{\prime}$ & 56.31921 & 24.53142 & 2 & $\mathrm{~s}$ & $4 \mathrm{pl}$ basalt & 130 & 52 & $\mathrm{P}$ & bended No. 3 \\
\hline 5 & Sheeted dikes & Zab' in & OM19-40 & No. 47 & 56.31921 & 24.53142 & & & 122 aphyric basalt & 284 & 63 & $\mathrm{P}$ & Boundary with No. 51 \\
\hline 5 & Sheeted dikes & Zab' in & OM19-40 & No. 51 & 56.31921 & 24.53142 & 2 & $\mathrm{~s}$ & 28 aphyric basalt & 284 & 63 & $\mathrm{P}$ & Boundary with No. 47 \\
\hline 5 & Sheeted dikes & Zab' in & OM19-40 & No. 64 & 56.31921 & 24.53142 & 2 & $\mathrm{~s}$ & 10 aphyric basalt & 292 & 80 & $\mathrm{P}$ & boundary No. $65 ; 1.5,2.5 \mathrm{~cm}$ branch \\
\hline 5 & Sheeted dikes & Zab' in & OM19-40 & No. 51 & 56.31921 & 24.53142 & 2 & $\mathrm{~s}$ & 28 aphyric basalt & 300 & 88 & $\mathrm{P}$ & Boundary with No. 50 \\
\hline 5 & Sheeted dikes & Zab' in & OM19-40 & No. 63 & 56.31921 & 24.53142 & 2 & $\mathrm{~s}$ & $19 \mathrm{cpx} \mathrm{pl} \mathrm{basalt}$ & 300 & 68 & $\mathrm{P}$ & partly cut by No. 62 \\
\hline
\end{tabular}




\begin{tabular}{|c|c|c|c|c|c|c|c|c|c|c|c|c|c|}
\hline \multirow[t]{2}{*}{ Swarm } & \multirow[t]{2}{*}{ Host/Screen } & \multirow[t]{2}{*}{ Area } & \multirow[t]{2}{*}{ Locality } & \multirow[t]{2}{*}{ ID } & \multirow[t]{2}{*}{ Long } & \multirow[t]{2}{*}{ Lat } & \multirow{2}{*}{$\begin{array}{c}\text { Two/One } \\
\text { sided } \\
1 / 2\end{array}$} & \multirow{2}{*}{$\begin{array}{c}\text { Type } \\
\text { S/M/C/B }\end{array}$} & \multirow[t]{2}{*}{ Thick $[\mathrm{cm}] \quad$ Rock type } & \multirow[t]{2}{*}{ Azimuth } & \multirow[t]{2}{*}{ Dip } & \multirow[t]{2}{*}{$\mathrm{L} / \mathrm{P}$} & \multirow[t]{2}{*}{ Note } \\
\hline & & & & & & & & & & & & & \\
\hline 5 & Sheeted dikes & $2 a b^{\prime}$ in & OM19-40 & No. 78 & 56.31921 & 24.53142 & 2 & $\mathrm{~s}$ & 2.5 aphyric basalt & 350 & 81 & $P$ & echelon \\
\hline 5 & Sheeted dikes & $2 a b^{\prime}$ in & OM19-40 & No. 57 & 56.31921 & 24.53142 & 2 & $\mathrm{~s}$ & $53 \mathrm{cpx}$ basalt & 352 & 84 & $\mathrm{P}$ & cut No. $58,59,60$ \\
\hline 5 & Sheeted dikes & Zab' in & OM19-40 & No. 19 & 56.31921 & 24.53142 & 1 & s & $59 \mathrm{cpx} \mathrm{pl} \mathrm{ol} \mathrm{basalt}$ & 353 & 71 & $\mathrm{P}$ & \\
\hline 5 & Sheeted dikes & $Z a b^{\prime}$ in & OM19-40 & No. 74 & 56.31921 & 24.53142 & 2 & $\mathrm{~s}$ & aphyric basalt & 353 & 86 & $\mathrm{P}$ & intrude into No. 73 \\
\hline 5 & Sheeted dikes & $Z a b^{\prime}$ in & OM19-40 & No. 80 & 56.31921 & 24.53142 & 2 & s & $56 \mathrm{cpx}$ basalt & 353 & 75 & $\mathrm{P}$ & \\
\hline 5 & Sheeted dikes & $Z a b^{\prime}$ in & OM19-40 & No. 61 & 56.31921 & 24.53142 & 2 & B & 6 aphyric basalt & 355 & 82 & $\mathrm{P}$ & cut No. $58,59,60,62$ '; banding $1.5,1.5,1,2 \mathrm{~cm}$ east to west \\
\hline 5 & Sheeted dikes & Zab' in & OM19-40 & No. 71 & 56.31921 & 24.53142 & 1 & $\mathrm{~s}$ & 36 aphyric basalt & 356 & 88 & $\mathrm{P}$ & cut 72,73 \\
\hline 5 & Sheeted dikes & $2 a b^{\prime}$ in & OM19-40 & No. 21 & 56.31921 & 24.53142 & 2 & $\mathrm{~s}$ & $4 \mathrm{cpx}$ basalt & 357 & 79 & $\mathrm{P}$ & cut No. $22,23,24$ \\
\hline 5 & Sheeted dikes & Zab' in & OM19-40 & No. 18 & 56.31921 & 24.53142 & 2 & $\mathrm{~s}$ & 11 aphyric basalt & 4 & 80 & $\mathrm{P}$ & intrude into No. 19 \\
\hline 5 & Sheeted dikes & $Z a b^{\prime}$ in & OM19-40 & No. 78 & 56.31921 & 24.53142 & 2 & $\mathrm{~s}$ & 3.5 aphyric basalt & 16 & 85 & $\mathrm{P}$ & echelon \\
\hline 5 & Sheeted dikes & Zab' in & OM19-40 & No. 66' & 56.31921 & 24.53142 & 2 & $\mathrm{~s}$ & 6 aphyric basalt & 161 & 28 & $\mathrm{P}$ & branch of No. 66 \\
\hline 5 & Sheeted dikes & $2 a b^{\prime}$ in & OM19-40 & No. 46 & 56.31921 & 24.53142 & 1 & $\mathrm{~s}$ & $121 \mathrm{cpx} \mathrm{pl} \mathrm{basalt}$ & 168 & 74 & $\mathrm{P}$ & cut 47,48 \\
\hline 5 & Sheeted dikes & $Z a b^{\prime}$ in & OM19-40 & No. 48 & 56.31921 & 24.53142 & 2 & $\mathrm{~s}$ & $15 \mathrm{cp} \times \mathrm{pl}$ basalt & 168 & 76 & $\mathrm{P}$ & \\
\hline 5 & Sheeted dikes & $Z a b^{\prime}$ in & OM19-40 & No. 25 & 56.31921 & 24.53142 & 1 & $\mathrm{~s}$ & $50 \mathrm{cpx} \mathrm{pl} \mathrm{basalt}$ & 170 & 74 & $\mathrm{P}$ & \\
\hline 5 & Sheeted dikes & $Z a b^{\prime}$ in & OM19-40 & No. 68 & 56.31921 & 24.53142 & 2 & $\mathrm{~s}$ & 6 aphyric basalt & 170 & 86 & $\mathrm{P}$ & cut No. 64 \\
\hline 5 & Sheeted dikes & $Z a b^{\prime}$ in & OM19-40 & No. 73 & 56.31921 & 24.53142 & 2 & $\mathrm{~s}$ & 14 aphyric basalt & 172 & 53 & $\mathrm{P}$ & intrude into No. 72 \\
\hline 5 & Sheeted dikes & $2 a b^{\prime}$ in & OM19-40 & No. 82 & 56.31921 & 24.53142 & 2 & $\mathrm{~s}$ & $49 \mathrm{cpx} \mathrm{pl} \mathrm{basalt}$ & 175 & 60 & $\mathrm{P}$ & \\
\hline 5 & Sheeted dikes & $Z a b^{\prime}$ in & OM19-40 & No. 75 & 56.31921 & 24.53142 & 2 & $\mathrm{~s}$ & $3 \mathrm{cpx}$ basalt & 176 & 80 & $\mathrm{P}$ & intrude into No. 76 \\
\hline 5 & Sheeted dikes & $Z a b^{\prime}$ in & OM19-40 & No. 66 & 56.31921 & 24.53142 & 2 & $\mathrm{~s}$ & 12 aphyric basalt & 177 & 71 & $\mathrm{P}$ & cut No. 67 \\
\hline 5 & Sheeted dikes & $2 a b^{\prime}$ in & OM19-40 & No. 72 & 56.31921 & 24.53142 & 1 & $\mathrm{~s}$ & $33 \mathrm{cpx}$ pl basalt & 180 & 76 & $\mathrm{P}$ & \\
\hline 5 & Sheeted dikes & $Z a b^{\prime}$ in & OM19-40 & No. 65 & 56.31921 & 24.53142 & 1 & $\mathrm{~s}$ & $40 \mathrm{cpx}$ pl basalt & 182 & 68 & $\mathrm{P}$ & boundary with No. 67 ; cut No. 66 \\
\hline 5 & Sheeted dikes & $Z a b^{\prime}$ in & OM19-40 & No. 39 & 56.31921 & 24.53142 & 2 & $\mathrm{~s}$ & $18 \mathrm{cpx}$ basalt & 188 & 72 & $\mathrm{P}$ & cut No. $34,36,42,43$ \\
\hline 5 & Sheeted dikes & $Z a b^{\prime}$ in & OM19-40 & No. 78 & 56.31921 & 24.53142 & 2 & $\mathrm{~s}$ & 3.5 aphyric basalt & 189 & 67 & $\mathrm{P}$ & echelon \\
\hline 5 & Sheeted dikes & $2 a b^{\prime}$ in & OM19-40 & No. 64 & 56.31921 & 24.53142 & 2 & $\mathrm{~s}$ & 2 aphyric basalt & 190 & 70 & $\mathrm{P}$ & \\
\hline 5 & Sheeted dikes & Zab' in & OM19-40 & No. 1 & 56.31921 & 24.53142 & 1 & $\mathrm{~s}$ & 13 aphyric basalt & 192 & 73 & $\mathrm{P}$ & cut by No. 3 \\
\hline 5 & Sheeted dikes & $Z a b^{\prime}$ in & OM19-40 & No. 3 & 56.31921 & 24.53142 & 2 & $\mathrm{~s}$ & $50 \mathrm{pl} \mathrm{basalt}$ & 196 & 70 & $\mathrm{P}$ & \\
\hline 5 & Sheeted dikes & $2 a b^{\prime}$ in & OM19-40 & No. 35 & 56.31921 & 24.53142 & 2 & $\mathrm{~s}$ & 5 aphyric basalt & 196 & 58 & $\mathrm{P}$ & cut No. 40,41 \\
\hline 5 & Sheeted dikes & $Z a b^{\prime}$ in & OM19-40 & No. 40 & 56.31921 & 24.53142 & 1 & $\mathrm{~s}$ & $47 \mathrm{cpx}$ pl basalt & 196 & 77 & $\mathrm{P}$ & \\
\hline 5 & Sheeted dikes & $Z a b^{\prime}$ in & OM19-40 & No. 2 & 56.31921 & 24.53142 & 1 & $\mathrm{~s}$ & $127 \mathrm{cpx}$ basalt & 198 & 84 & $\mathrm{P}$ & w/8 $\mathrm{cm}$ wide branch; cut by No. 3 \\
\hline 5 & Sheeted dikes & Zab' in & OM19-40 & No. 10 & 56.31921 & 24.53142 & 2 & $\mathrm{~s}$ & 2.5 aphyric basalt & 198 & 84 & $\mathrm{P}$ & Included in No. 4 \\
\hline 5 & Sheeted dikes & $2 a b^{\prime}$ in & OM19-40 & No. 76 & 56.31921 & 24.53142 & 1 & $\mathrm{~s}$ & $29 \mathrm{cpx} \mathrm{pl} \mathrm{basalt}$ & 198 & 50 & $\mathrm{P}$ & \\
\hline 5 & Sheeted dikes & $2 a b^{\prime}$ in & OM19-40 & No. 6 & 56.31921 & 24.53142 & 2 & $\mathrm{~s}$ & 14 aphyric basalt & 202 & 63 & $\mathrm{P}$ & \\
\hline 5 & Sheeted dikes & Zab' in & OM19-40 & No. 36 & 56.31921 & 24.53142 & 2 & $\mathrm{~s}$ & 8 aphyric basalt & 202 & 56 & $\mathrm{P}$ & cut No. 37 \\
\hline 5 & Sheeted dikes & Zab' in & OM19-40 & No. 49 & 56.31921 & 24.53142 & 2 & $\mathrm{~s}$ & $78 \mathrm{cpx} \mathrm{pl} \mathrm{basalt}$ & 202 & 70 & $\mathrm{P}$ & \\
\hline 5 & Sheeted dikes & $2 a b^{\prime}$ in & OM19-40 & No. 53a & 56.31921 & 24.53142 & 1 & M & 44 aphyric basalt & 202 & 68 & $\mathrm{P}$ & \\
\hline 5 & Sheeted dikes & $Z a b^{\prime}$ in & OM19-40 & No. 23 & 56.31921 & 24.53142 & 2 & $\mathrm{~s}$ & $12 \mathrm{cpx} \mathrm{pl} \mathrm{basalt}$ & 203 & 78 & $\mathrm{P}$ & \\
\hline 5 & Sheeted dikes & Zab' in & OM19-40 & No. 7 & 56.31921 & 24.53142 & 2 & $\mathrm{~s}$ & 42 aphyric basalt & 206 & 83 & $\mathrm{P}$ & Sample \\
\hline 5 & Sheeted dikes & $2 a b^{\prime}$ in & OM19-40 & No. 11 & 56.31921 & 24.53142 & 2 & $\mathrm{~s}$ & 3 aphyric basalt & 206 & 70 & $\mathrm{P}$ & Included in No. 4 \\
\hline 5 & Sheeted dikes & $2 a b^{\prime}$ in & OM19-40 & No. 9 & 56.31921 & 24.53142 & 2 & $\mathrm{~s}$ & 3 aphyric basalt & 208 & 63 & $\mathrm{P}$ & Included in No. 4 \\
\hline 5 & Sheeted dikes & $Z a b^{\prime}$ in & OM19-40 & No. 27 & 56.31921 & 24.53142 & 2 & $\mathrm{~s}$ & 4 aphyric basalt & 208 & 52 & $\mathrm{P}$ & \\
\hline 5 & Sheeted dikes & $Z a b^{\prime}$ in & OM19-40 & No. 29 & 56.31921 & 24.53142 & 1 & $\mathrm{~s}$ & 35 aphyric basalt & 208 & 51 & $\mathrm{P}$ & cut No. 30, 31 \\
\hline 5 & Sheeted dikes & $2 a b^{\prime}$ in & OM19-40 & No. 31 & 56.31921 & 24.53142 & 1 & $\mathrm{~s}$ & 29 aphyric basalt & 208 & 49 & $\mathrm{P}$ & \\
\hline 5 & Sheeted dikes & $Z a b^{\prime}$ in & OM19-40 & No. 51' & 56.31921 & 24.53142 & 1 & $\mathrm{~s}$ & 66 aphyric basalt & 209 & 60 & $\mathrm{P}$ & \\
\hline 5 & Sheeted dikes & $2 a b^{\prime}$ in & OM19-40 & No. 12 & 56.31921 & 24.53142 & 2 & $\mathrm{~s}$ & 13 aphyric basalt & 210 & 62 & $\mathrm{P}$ & \\
\hline 5 & Sheeted dikes & Zab' in & OM19-40 & No. 43 & 56.31921 & 24.53142 & 2 & $\mathrm{~s}$ & $12 \mathrm{cpx}$ basalt & 210 & 47 & $\mathrm{P}$ & intrude into No. 42 ; cut No. 44 \\
\hline 5 & Sheeted dikes & $Z a b$ ' in & OM19-40 & No. 56 & 56.31921 & 24.53142 & 2 & $\mathrm{~s}$ & $124 \mathrm{cpx} \mathrm{pl} \mathrm{basalt}$ & 210 & 56 & $\mathrm{P}$ & \\
\hline 5 & Sheeted dikes & $Z a b^{\prime}$ in & OM19-40 & No. 26 & 56.31921 & 24.53142 & 2 & $\mathrm{~s}$ & $12 \mathrm{pl}$ basalt & 212 & 68 & $\mathrm{P}$ & \\
\hline
\end{tabular}




\begin{tabular}{|c|c|c|c|c|c|c|c|c|c|c|c|c|c|}
\hline \multirow[t]{2}{*}{ Swarm } & \multirow[t]{2}{*}{ Host/Screen } & \multirow[t]{2}{*}{ Area } & \multirow[t]{2}{*}{ Locality } & \multirow[t]{2}{*}{ ID } & \multirow[t]{2}{*}{ Long } & \multirow[t]{2}{*}{ Lat } & \multirow{2}{*}{$\begin{array}{c}\text { Two/One } \\
\text { sided } \\
1 / 2\end{array}$} & \multirow{2}{*}{$\begin{array}{c}\text { Type } \\
\text { S/M/C/B }\end{array}$} & \multirow[t]{2}{*}{ Thick $[\mathrm{cm}] \quad$ Rock type } & \multirow[t]{2}{*}{ Azimuth } & \multirow[t]{2}{*}{ Dip } & \multirow[t]{2}{*}{$L / P$} & \multirow[t]{2}{*}{ Note } \\
\hline & & & & & & & & & & & & & \\
\hline 5 & Sheeted dikes & $Z a b^{\prime}$ in & OM19-40 & No. 26' & 56.31921 & 24.53142 & 2 & $\mathrm{~s}$ & $5 \mathrm{pl}$ basalt & 212 & 64 & $\mathrm{P}$ & branch of No. 26; intrude into No. 28 \\
\hline 5 & Sheeted dikes & $Z a b^{\prime}$ in & OM19-40 & No. 30 & 56.31921 & 24.53142 & 2 & $\mathrm{~s}$ & $7.5 \mathrm{cpx} \mathrm{pl}$ basalt & 212 & 53 & $\mathrm{P}$ & \\
\hline 5 & Sheeted dikes & $Z_{a b}$ in & OM19-40 & No. 37 & 56.31921 & 24.53142 & 1 & $\mathrm{~s}$ & $31 \mathrm{cpxpl}$ basalt & 212 & 33 & $\mathrm{P}$ & \\
\hline 5 & Sheeted dikes & Zab' in & OM19-40 & No. 59 & 56.31921 & 24.53142 & 2 & $\mathrm{~s}$ & 25 aphyric basalt & 212 & 55 & $\mathrm{P}$ & intrude into No. 60 \\
\hline 5 & Sheeted dikes & $Z a b^{\prime}$ in & OM19-40 & No. 5 & 56.31921 & 24.53142 & 2 & $\mathrm{~s}$ & 12 aphyric basalt & 215 & 57 & $\mathrm{P}$ & \\
\hline 5 & Sheeted dikes & $Z a b^{\prime}$ in & OM19-40 & No. 70 & 56.31921 & 24.53142 & 2 & $\mathrm{~s}$ & $10 \mathrm{cpx}$ ol basalt & 215 & 72 & $\mathrm{P}$ & \\
\hline 5 & Sheeted dikes & $Z a b^{\prime}$ in & OM19-40 & No. 82 & 56.31921 & 24.53142 & 2 & $\mathrm{~s}$ & $9.5 \mathrm{cpx} \mathrm{pl}$ basalt & 215 & 58 & $\mathrm{P}$ & branch \\
\hline 5 & Sheeted dikes & $Z a b^{\prime}$ in & OM19-40 & No. 17 & 56.31921 & 24.53142 & 2 & $\mathrm{~s}$ & $38 \mathrm{cpx}$ basalt & 216 & 60 & $\mathrm{P}$ & \\
\hline 5 & Sheeted dikes & $Z a b^{\prime}$ in & OM19-40 & No. 4 & 56.31921 & 24.53142 & & & $80 \mathrm{cpx} \mathrm{pl} \mathrm{basalt}$ & 218 & 67 & $\mathrm{P}$ & \\
\hline 5 & Sheeted dikes & $Z a b^{\prime}$ in & OM19-40 & No. 52 & 56.31921 & 24.53142 & 1 & $\mathrm{~s}$ & $27 \mathrm{cpx} \mathrm{pl} \mathrm{basalt}$ & 218 & 38 & $\mathrm{P}$ & \\
\hline 5 & Sheeted dikes & Zab' in & OM19-40 & No. 16 & 56.31921 & 24.53142 & 2 & $\mathrm{~s}$ & 12.5 aphyric basalt & 220 & 61 & $\mathrm{P}$ & \\
\hline 5 & Sheeted dikes & $Z a b^{\prime}$ in & OM19-40 & No. 54 & 56.31921 & 24.53142 & 2 & $\mathrm{~s}$ & $120 \mathrm{cpx} \mathrm{pl} \mathrm{basalt}$ & 220 & 64 & $\mathrm{P}$ & cut No. $51^{\prime}, 52,53,55$ \\
\hline 5 & Sheeted dikes & $Z a b^{\prime}$ in & OM19-40 & No. 58 & 56.31921 & 24.53142 & 2 & $\mathrm{~s}$ & $248 \mathrm{cpx} \mathrm{pl} \mathrm{basalt}$ & 220 & 47 & $\mathrm{P}$ & \\
\hline 5 & Sheeted dikes & $Z a b^{\prime}$ in & OM19-40 & No. 13 & 56.31921 & 24.53142 & 1 & $\mathrm{~s}$ & cpx basalt & 224 & 53 & $\mathrm{P}$ & \\
\hline 5 & Sheeted dikes & $Z_{a b}$ in & OM19-40 & No. 22 & 56.31921 & 24.53142 & 2 & $\mathrm{~s}$ & $37 \mathrm{cpx}$ basalt & 224 & 53 & $\mathrm{P}$ & \\
\hline 5 & Sheeted dikes & $Z a b^{\prime}$ in & OM19-40 & No. 15 & 56.31921 & 24.53142 & 2 & $\mathrm{~s}$ & 48 aphyric basalt & 225 & 60 & $\mathrm{P}$ & \\
\hline 5 & Sheeted dikes & $Z a b^{\prime}$ in & OM19-40 & No. 50 & 56.31921 & 24.53142 & 1 & $\mathrm{~s}$ & $19 \mathrm{cpx}$ basalt & 226 & 50 & $\mathrm{P}$ & boundary with No. 49 \\
\hline 5 & Sheeted dikes & $Z a b^{\prime}$ in & OM19-40 & No. 8 & 56.31921 & 24.53142 & 1 & $\mathrm{~s}$ & 57 aphyric basalt & 228 & 73 & $\mathrm{P}$ & \\
\hline 5 & Sheeted dikes & $Z a b^{\prime}$ in & OM19-40 & No. 44 & 56.31921 & 24.53142 & 2 & $\mathrm{~s}$ & 2 aphyric basalt & 230 & 47 & $\mathrm{P}$ & \\
\hline 5 & Sheeted dikes & $Z a b^{\prime}$ in & OM19-40 & No. 41 & 56.31921 & 24.53142 & 2 & $\mathrm{~s}$ & $7 \mathrm{cpxpl}$ basalt & 231 & 73 & $\mathrm{P}$ & cut No. 40 \\
\hline 5 & Sheeted dikes & $Z a b^{\prime}$ in & OM19-40 & No. $62^{\prime}$ & 56.31921 & 24.53142 & 2 & $\mathrm{~s}$ & aphyric basalt & 232 & 48 & $\mathrm{P}$ & branch of No. 62 \\
\hline 5 & Sheeted dikes & $Z a b^{\prime}$ in & OM19-40 & No. 32 & 56.31921 & 24.53142 & 2 & $\mathrm{~s}$ & $5 \mathrm{cpx}$ pl basalt & 238 & 45 & $\mathrm{P}$ & Included in No. 33 \\
\hline 5 & Sheeted dikes & $Z a b^{\prime}$ in & OM19-40 & No. 20 & 56.31921 & 24.53142 & 2 & $\mathrm{~s}$ & 2 aphyric basalt & 257 & 40 & $\mathrm{P}$ & Included in No. 19 \\
\hline 5 & Sheeted dikes & $Z a b^{\prime}$ in & OM19-40 & No. 62 & 56.31921 & 24.53142 & 2 & B & 30 aphyric basalt & 314 & 68 & $\mathrm{P}$ & \\
\hline 5 & Sheeted dikes & $Z a b^{\prime}$ in & OM19-40 & No. $7^{\prime}$ & 56.31921 & 24.53142 & & & dolerite & & & & screen \\
\hline 5 & Sheeted dikes & $Z a b^{\prime}$ in & OM19-40 & No. 14 & 56.31921 & 24.53142 & & & 35 dolerite & & & & screen \\
\hline 5 & Sheeted dikes & $Z a b^{\prime}$ in & OM19-40 & No. 24 & 56.31921 & 24.53142 & & & 17 & & & & screen \\
\hline 5 & Sheeted dikes & $Z a b^{\prime}$ in & OM19-40 & No. 28 & 56.31921 & 24.53142 & 2 & $\mathrm{~s}$ & 62 aphyric basalt & & & & $3 \mathrm{~mm} \mathrm{cpx} \mathrm{in} \mathrm{core}$ \\
\hline 5 & Sheeted dikes & $Z a b^{\prime}$ in & OM19-40 & No. $28^{\prime}$ & 56.31921 & 24.53142 & & & 5 aphyric basalt & & & & Inclusion patch of No. 28 \\
\hline 5 & Sheeted dikes & $Z a b^{\prime}$ in & OM19-40 & No. 33 & 56.31921 & 24.53142 & 2 & $\mathrm{~s}$ & $29 \mathrm{cpx}$ basalt & & & & cut by No. 28 \\
\hline 5 & Sheeted dikes & $Z a b^{\prime}$ in & OM19-40 & No. 34 & 56.31921 & 24.53142 & & & 38.5 dolerite & & & & screen \\
\hline 5 & Sheeted dikes & $Z a b^{\prime}$ in & OM19-40 & No. 38 & 56.31921 & 24.53142 & & & dolerite & & & & screen \\
\hline 5 & Sheeted dikes & $Z a b^{\prime}$ in & OM19-40 & No. 42 & 56.31921 & 24.53142 & & & 50 dolerite & & & & screen \\
\hline 5 & Sheeted dikes & $Z a b^{\prime}$ in & OM19-40 & No. 45 & 56.31921 & 24.53142 & & & 78 dolerite & & & & screen \\
\hline 5 & Sheeted dikes & $2 a b^{\prime}$ in & OM19-40 & No. $53 \mathrm{~b}$ & 56.31921 & 24.53142 & 1 & M & 57 aphyric basalt & & & & \\
\hline 5 & Sheeted dikes & $Z a b^{\prime}$ in & OM19-40 & No. 55 & 56.31921 & 24.53142 & & & 62 aphyric dolerite & & & & screen \\
\hline 5 & Sheeted dikes & Zab' in & OM19-40 & No. 60 & 56.31921 & 24.53142 & & & 85 aphyric dolerite & & & & \\
\hline 5 & Sheeted dikes & $Z a b^{\prime}$ in & OM19-40 & No. 67 & 56.31921 & 24.53142 & & & aphyric dolerite & & & & screen \\
\hline 5 & Sheeted dikes & $Z a b^{\prime}$ in & OM19-40 & No. 69 & 56.31921 & 24.53142 & & & 37 aphyric basalt & & & & screen \\
\hline 5 & Sheeted dikes & $Z a b^{\prime}$ in & OM19-40 & No. 77 & 56.31921 & 24.53142 & & & $9 \mathrm{cpx}$ pl basalt & & & & screen \\
\hline 5 & Sheeted dikes & $Z a b^{\prime}$ in & OM19-40 & No. 79 & 56.31921 & 24.53142 & & & 66 aphyric basalt & & & & screen \\
\hline 5 & Sheeted dikes & Zab' in & OM19-40 & No. 81 & 56.31921 & 24.53142 & & & 55 aphyric basalt & & & & screen \\
\hline 5 & Late intrusives & Zab' in & OM19-58 & No. 6 & 56.29767 & 24.53815 & 2 & s & 26 & 0 & 82 & $\mathrm{p}$ & \\
\hline 5 & Late intrusives & Zab' in & OM19-58 & No. 5 & 56.29767 & 24.53815 & 2 & $\mathrm{~s}$ & 35 & 2 & 86 & $\mathrm{P}$ & \\
\hline 5 & Late intrusives & $Z a b^{\prime}$ in & OM19-58 & No. 1 & 56.29767 & 24.53815 & & & & 4 & 86 & $\mathrm{P}$ & \\
\hline 5 & Late intrusives & $Z a b^{\prime}$ in & OM19-58 & No. 4 & 56.29767 & 24.53815 & & & & 8 & 72 & $\mathrm{P}$ & cut No. 3 \\
\hline
\end{tabular}




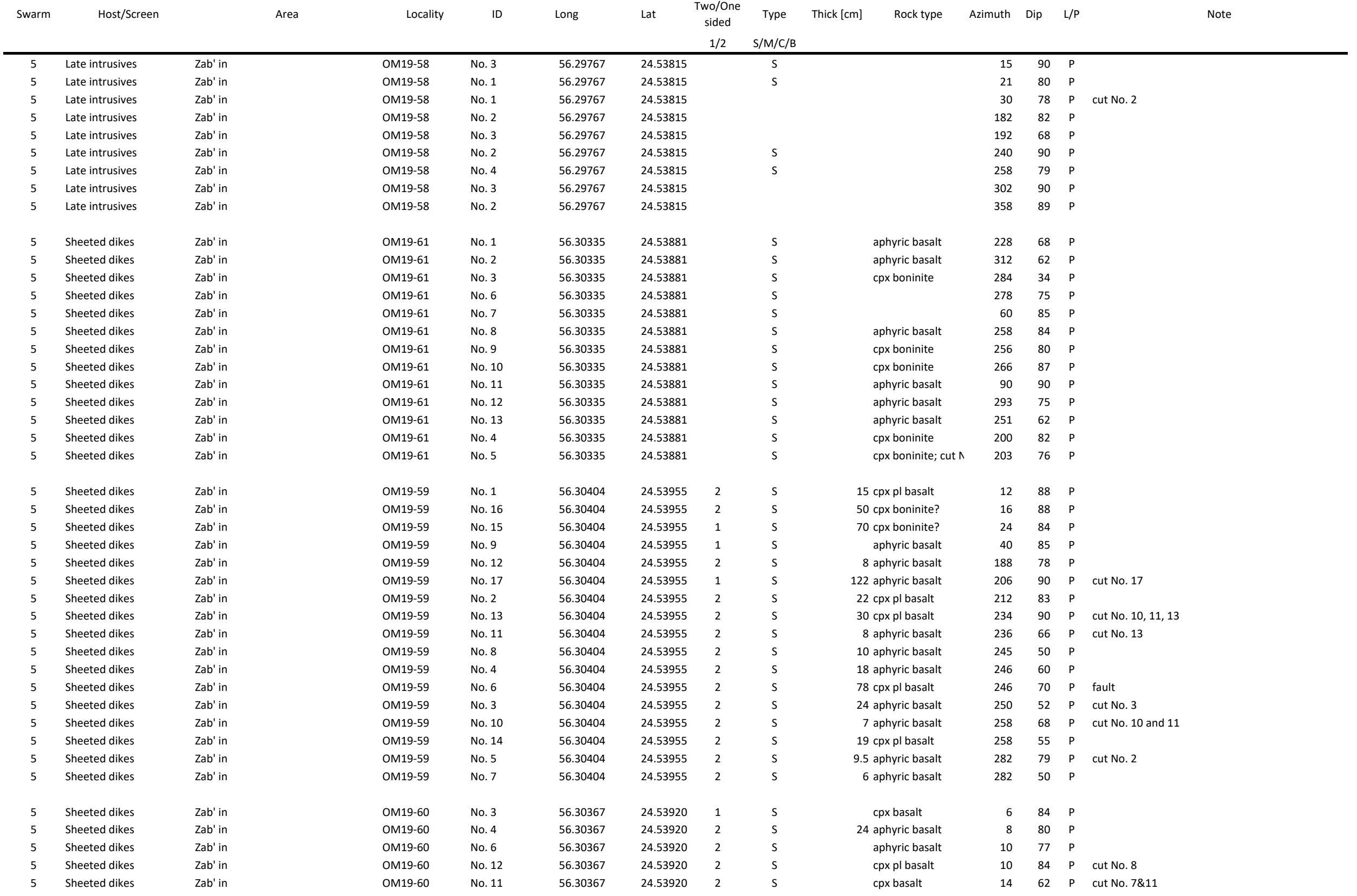




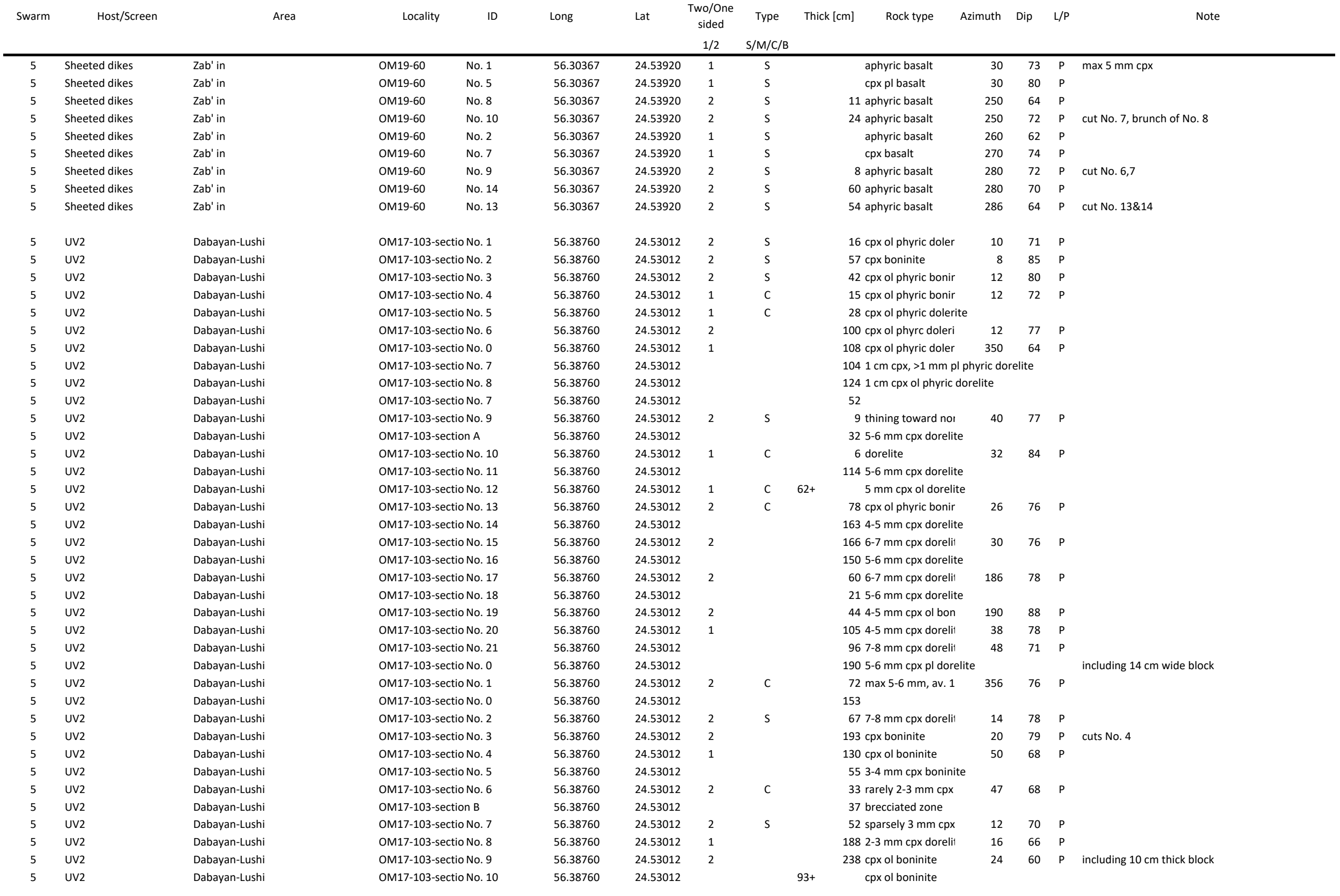




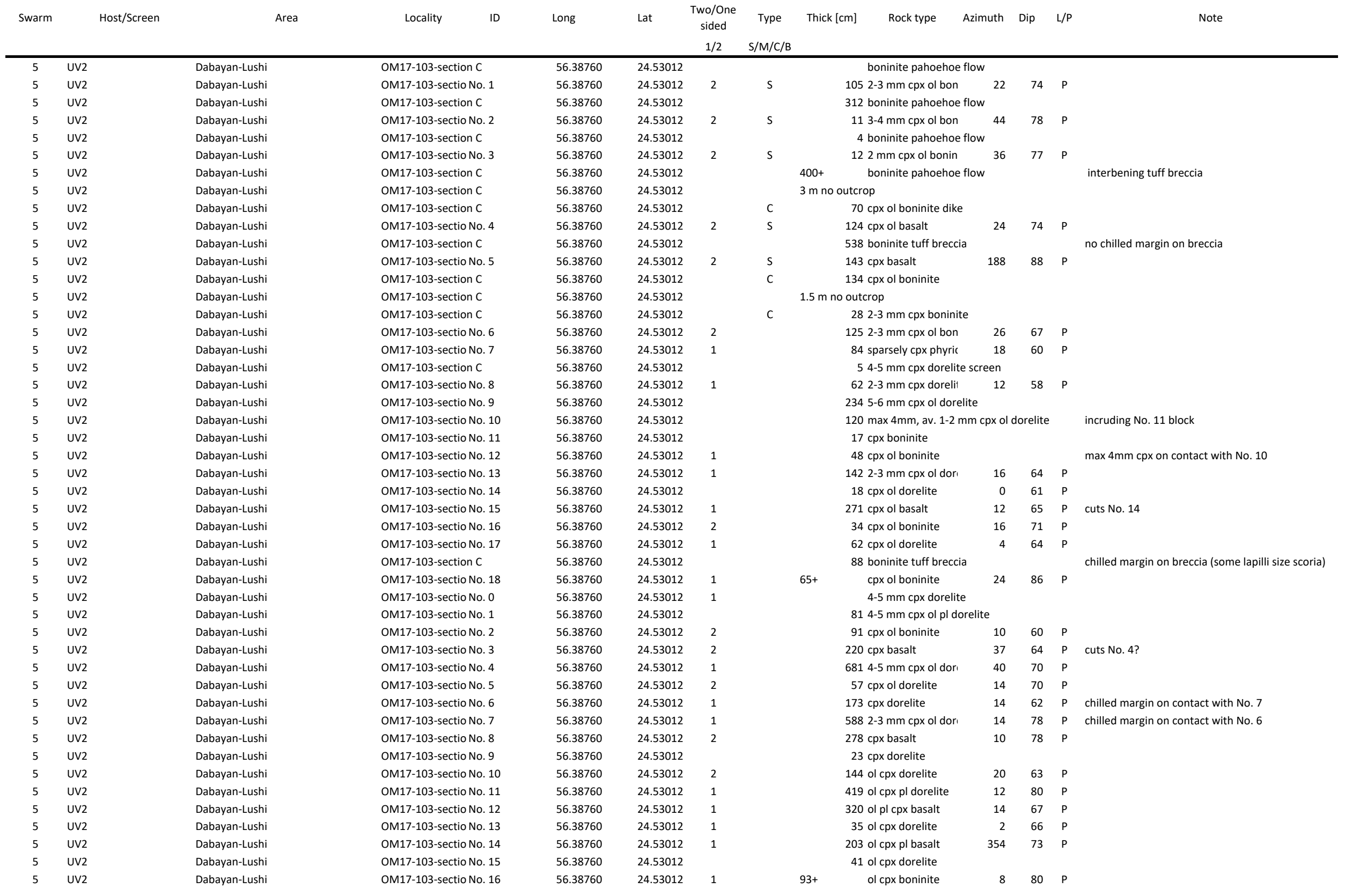


Latitude Longitude

Original [wt\%]

Anhydrous [wt\%]

ID

$\begin{array}{llll}56.29953 & 24.87236 & \mathrm{TH}\end{array}$

$\begin{array}{llll}\text { OM19-35-22 } & 56.29968 & 24.87236 & \text { TH }\end{array}$

OM19-35-24 $56.29968 \quad 24.87236 \quad$ TH

OM19-35-81 $56.29998 \quad 24.87231 \quad$ TH

OM19-35-96 $56.30000 \quad 24.87225 \quad$ TH

$\begin{array}{llll}\text { OM19-35-106 } & 56.30000 & 24.87225 & \text { TH }\end{array}$

\section{V2 dike swarm}

$\begin{array}{llll}\text { OM19-43-03 } 56.35506 & 24.69766 & \text { TH }\end{array}$

OM19-43-10 $56.35506 \quad 24.69766 \quad$ TH

OM19-45-01 56.32298 24.71308 BN

$\begin{array}{llll}\text { OM19-45-12 } & 56.32298 & 24.71308 & \text { BN }\end{array}$

$\begin{array}{llll}\text { OM19-45-32 } & 56.32298 & 24.71308 & \text { BN }\end{array}$

$\begin{array}{llll}\text { OM19-45-41 } & 56.32298 & 24.71308 & \text { BN }\end{array}$

$\begin{array}{llll}\text { OM19-46 } & 56.32424 & 24.71875 & \text { TH }\end{array}$

$\begin{array}{llll}\text { OM19-47-05core } & 56.32091 & 24.71845 & \text { BN }\end{array}$

$\begin{array}{llll}\text { OM19-23-06 } & 56.34674 & 24.62944 & B N\end{array}$

$\begin{array}{llll}\text { OM19-23-08 } & 56.34674 & 24.62944 & \text { BN }\end{array}$

$\begin{array}{llll}\text { OM19-23-09 } & 56.34674 & 24.62944 & \text { BN }\end{array}$

$\begin{array}{llll}\text { OM19-23-10 } & 56.34674 & 24.62944\end{array}$

$\begin{array}{llll}\text { OM19-24-03 } & 56.34666 & 24.62994 & \text { TH }\end{array}$

$\begin{array}{llll}\text { OM19-24-07 } & 56.34666 & 24.62994 & \text { TH }\end{array}$

$\begin{array}{llll}\text { OM19-24-20 } & 56.34666 & 24.62994 & \text { BN }\end{array}$

$\begin{array}{llll}\text { OM19-25-03 } 56.33172 & 24.62788 & \text { BN }\end{array}$

$\begin{array}{llll} & 56.33172 & 24.62788 & -62788 \text { BN }\end{array}$

OM19-26-00 $56.33168 \quad 24.62819$ (J) BN

$\begin{array}{llll}\text { OM19-26-03 } & 56.33168 & 24.62819 & \text { BN }\end{array}$

$\begin{array}{llll}\text { OM19-26-09 } & 56.33168 & 24.62819 & \text { BN }\end{array}$

$\begin{array}{llll}\text { OM19-32-01 } & 56.37437 & 24.62359 & \text { BN }\end{array}$

$\begin{array}{llll}\text { OM19-32-03 } & 56.37437 & 24.62359 & \text { BN }\end{array}$

$\begin{array}{llll}\text { OM19-34-01 } & 56.37515 & 24.62406 & B N\end{array}$

$\begin{array}{llll}\text { OM19-34-07 } & 56.37515 & 24.62406 & \text { BN }\end{array}$

$\begin{array}{llll}\text { OM19-14-2 } & 56.37344 & 24.58322 & \text { BN }\end{array}$

$\begin{array}{llll}\text { OM19-14-6 } & 56.37340 & 24.58241 & \text { BN }\end{array}$

$\begin{array}{llll}\text { OM19-14-8 } & 56.37338 & 24.58239 & \text { BN }\end{array}$

$\begin{array}{llll}\text { OM19-14-15a } & 56.37356 & 24.58223 & B N\end{array}$

$\begin{array}{llll}\text { OM19-14-15b } 56.37356 & 24.58223 & \text { BN }\end{array}$

OM19-14-16 $56.37354 \quad 24.58225 \quad$ BN

$\begin{array}{llll}\text { OM19-19-8 } & 56.53732 & 24.17367 & \text { BN }\end{array}$

$\begin{array}{llll}\text { OM19-19-9 } & 56.53751 & 24.17388\end{array}$ $\begin{array}{llllllllllll}\text { V1 dike } & 55.60 & 1.85 & 13.53 & 13.69 & 0.16 & 4.74 & 5.16 & 4.77 & 0.06 & 0.16 & 98.35\end{array}$ $\begin{array}{llllllllllll}\text { V1 dike } & 50.07 & 1.92 & 15.55 & 14.42 & 0.20 & 5.50 & 6.89 & 4.60 & 0.10 & 0.17 & 97.97\end{array}$

$\begin{array}{llllllllllll}\text { V1 dike } & 55.06 & 2.08 & 12.48 & 14.74 & 0.18 & 4.31 & 5.77 & 4.93 & 0.06 & 0.17 & 98.31\end{array}$

$\begin{array}{llllllllllll}\text { V1 dike } & 53.78 & 1.78 & 14.67 & 13.40 & 0.18 & 5.96 & 6.52 & 3.02 & 0.06 & 0.14 & 98.18\end{array}$

$\begin{array}{llllllllllll}\text { V1 dike } & 51.69 & 1.47 & 15.37 & 12.29 & 0.19 & 7.18 & 8.42 & 2.70 & 0.06 & 0.11 & 98.25\end{array}$

$\begin{array}{lllllllllllll}\text { V1 dike } & 56.37 & 1.63 & 14.52 & 12.80 & 0.17 & 5.10 & 4.22 & 4.52 & 0.24 & 0.19 & 98.48\end{array}$

$\begin{array}{llllllllllll}\text { SMW-1 } & 52.31 & 1.74 & 15.38 & 12.48 & 0.20 & 5.51 & 8.26 & 3.41 & 0.15 & 0.20 & 98.38\end{array}$ $\begin{array}{llllllllllll}\text { SMW-1 } & 58.39 & 1.57 & 14.30 & 11.83 & 0.22 & 3.50 & 4.60 & 4.72 & 0.09 & 0.36 & 98.39\end{array}$

$\begin{array}{llllllllllll}\text { SMW-1 } & 46.71 & 0.20 & 14.68 & 6.79 & 0.12 & 9.70 & 21.43 & 0.08 & 0.01 & 0.01 & 99.05\end{array}$

$\begin{array}{llllllllllll}\text { SMW-1 } & 53.04 & 0.14 & 2.82 & 5.68 & 0.12 & 20.03 & 17.45 & 0.20 & 0.02 & 0.00 & 98.94\end{array}$

$\begin{array}{llllllllllll}\text { SMW-1 } & 45.20 & 0.05 & 1.65 & 10.50 & 0.17 & 35.89 & 5.57 & 0.08 & 0.02 & 0.00 & 98.07\end{array}$

$\begin{array}{llllllllllll}\text { SMW-1 } & 44.56 & 0.05 & 1.89 & 11.17 & 0.18 & 35.98 & 4.89 & 0.10 & 0.02 & 0.00 & 97.72\end{array}$

$\begin{array}{llllllllllll}\text { SMW-1 } & 51.34 & 1.23 & 15.30 & 10.96 & 0.21 & 6.46 & 10.04 & 3.69 & 0.10 & 0.11 & 98.34\end{array}$

$\begin{array}{llllllllllll}\text { SMW-1 } & 53.35 & 0.55 & 13.08 & 10.63 & 0.17 & 9.81 & 10.39 & 1.30 & 0.06 & 0.02 & 98.30\end{array}$

$\begin{array}{llllllllllll}\text { SMW-3 } & 51.53 & 0.18 & 10.74 & 9.44 & 0.22 & 13.96 & 11.41 & 1.22 & 0.11 & 0.01 & 97.88\end{array}$

$\begin{array}{llllllllllll}\text { SMW-3 } & 55.51 & 0.32 & 14.97 & 9.49 & 0.15 & 7.42 & 10.05 & 1.38 & 0.15 & 0.02 & 98.50\end{array}$

$\begin{array}{llllllllllll}\text { SMW-3 } & 52.83 & 0.25 & 13.65 & 8.16 & 0.17 & 10.71 & 11.78 & 1.43 & 0.10 & 0.01 & 98.26\end{array}$

$\begin{array}{llllllllllll}\text { SMW-3 } & 53.93 & 0.23 & 11.60 & 8.51 & 0.16 & 12.24 & 12.05 & 0.61 & 0.04 & 0.01 & 98.53\end{array}$

$\begin{array}{llllllllllll}\text { SMW-3 } & 51.80 & 1.31 & 14.77 & 11.93 & 0.11 & 6.25 & 8.99 & 4.02 & 0.12 & 0.11 & 98.21\end{array}$

$\begin{array}{llllllllllll}\text { SMW-3 } & 51.15 & 1.80 & 14.61 & 14.45 & 0.21 & 6.18 & 6.23 & 4.75 & 0.09 & 0.19 & 98.22\end{array}$

$\begin{array}{llllllllllll}\text { SMW-3 } & 52.25 & 0.26 & 14.33 & 10.19 & 0.21 & 8.98 & 10.28 & 2.65 & 0.15 & 0.01 & 98.28\end{array}$

$\begin{array}{lllllllllllll}\text { SMW-3 } & 54.28 & 0.24 & 13.29 & 8.93 & 0.17 & 9.94 & 11.43 & 1.23 & 0.08 & 0.01 & 98.70\end{array}$

$\begin{array}{llllllllllll}\text { SMW-3 } & 56.31 & 0.40 & 15.67 & 10.95 & 0.19 & 4.94 & 9.09 & 2.04 & 0.08 & 0.02 & 98.60\end{array}$

$\begin{array}{llllllllllll}\text { SMW-3 } & 48.53 & 0.19 & 17.11 & 5.55 & 0.10 & 11.14 & 15.71 & 1.07 & 0.02 & 0.01 & 98.87\end{array}$

$\begin{array}{llllllllllll}\text { SMW-3 } & 55.39 & 0.32 & 15.89 & 9.94 & 0.19 & 5.99 & 10.48 & 1.37 & 0.06 & 0.01 & 98.64\end{array}$

$\begin{array}{llllllllllll}\text { SMW-3 } & 55.36 & 0.32 & 14.01 & 9.56 & 0.15 & 8.14 & 8.91 & 2.85 & 0.15 & 0.02 & 98.50\end{array}$

$\begin{array}{lllllllllllll}\text { SMW-3 } & 60.15 & 0.52 & 14.09 & 9.93 & 0.12 & 3.45 & 8.89 & 2.02 & 0.03 & 0.03 & 98.23\end{array}$

$\begin{array}{llllllllllll}\text { SMW-3 } & 56.61 & 0.48 & 15.65 & 9.91 & 0.19 & 6.68 & 4.63 & 4.90 & 0.27 & 0.03 & 98.34\end{array}$

$\begin{array}{llllllllllll}\text { SMW-3 } & 64.37 & 0.54 & 12.30 & 10.34 & 0.15 & 4.45 & 3.16 & 4.04 & 0.06 & 0.03 & 98.39\end{array}$

$\begin{array}{llllllllllll}\text { SMW-3 } & 59.68 & 0.63 & 14.17 & 11.64 & 0.17 & 4.77 & 4.80 & 3.27 & 0.16 & 0.03 & 98.15\end{array}$

$\begin{array}{llllllllllll}\text { SMW-4 } & 57.70 & 0.44 & 14.82 & 10.58 & 0.15 & 5.07 & 4.83 & 4.08 & 1.91 & 0.02 & 98.54\end{array}$

$\begin{array}{llllllllllll}\text { SMW-4 } & 58.21 & 0.45 & 14.45 & 10.79 & 0.14 & 4.22 & 6.68 & 3.98 & 0.57 & 0.02 & 98.43\end{array}$

$\begin{array}{llllllllllll}\text { SMW-4 } & 57.53 & 0.44 & 14.44 & 11.06 & 0.15 & 4.12 & 7.45 & 3.96 & 0.59 & 0.02 & 98.65\end{array}$

$\begin{array}{lllllllllllll}\text { SMW-4 } & 57.51 & 0.44 & 13.99 & 10.70 & 0.17 & 6.04 & 8.97 & 1.44 & 0.57 & 0.02 & 98.77\end{array}$

$\begin{array}{llllllllllll}\text { SMW-4 } & 55.90 & 0.31 & 12.12 & 9.32 & 0.16 & 9.81 & 10.18 & 1.24 & 0.50 & 0.02 & 98.63\end{array}$

$\begin{array}{llllllllllll}\text { SMW-4 } & 55.99 & 0.43 & 14.33 & 10.95 & 0.15 & 5.28 & 8.06 & 1.96 & 2.35 & 0.02 & 98.42\end{array}$

$\begin{array}{llllllllllll}\text { SMW-4 } & 55.79 & 0.25 & 10.65 & 9.48 & 0.25 & 12.02 & 8.49 & 2.26 & 0.11 & 0.02 & 98.35\end{array}$

$\begin{array}{llllllrlllll}\text { SMW-4 } & 64.40 & 1.11 & 14.24 & 8.37 & 0.11 & 2.17 & 2.97 & 6.05 & 0.03 & 0.21 & 98.82\end{array}$

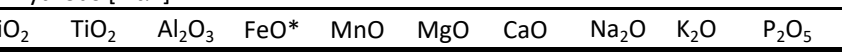

$\begin{array}{llllllllll}56.53 & 1.88 & 13.76 & 12.52 & 0.17 & 4.82 & 5.25 & 4.84 & 0.06 & 0.17\end{array}$

$\begin{array}{lllllllllll}51.10 & 1.96 & 15.87 & 13.25 & 0.20 & 5.62 & 7.03 & 4.69 & 0.10 & 0.17\end{array}$

$\begin{array}{llllllllll}56.01 & 2.11 & 12.70 & 13.49 & 0.18 & 4.38 & 5.87 & 5.01 & 0.06 & 0.18\end{array}$

$\begin{array}{llllllllll}54.78 & 1.82 & 14.95 & 12.28 & 0.19 & 6.07 & 6.64 & 3.08 & 0.06 & 0.14\end{array}$

$\begin{array}{llllllllll}52.61 & 1.50 & 15.65 & 11.26 & 0.19 & 7.31 & 8.57 & 2.74 & 0.06 & 0.12\end{array}$

$\begin{array}{lllllllllll}57.24 & 1.66 & 14.75 & 11.69 & 0.17 & 5.18 & 4.28 & 4.59 & 0.24 & 0.19\end{array}$

$\begin{array}{llllllllll}53.16 & 1.77 & 15.63 & 11.42 & 0.20 & 5.60 & 8.40 & 3.46 & 0.15 & 0.20\end{array}$

$\begin{array}{lllllllllll}59.34 & 1.59 & 14.54 & 10.82 & 0.22 & 3.56 & 4.68 & 4.79 & 0.10 & 0.37\end{array}$

$\begin{array}{llllllllll}47.16 & 0.20 & 14.82 & 6.17 & 0.12 & 9.79 & 21.64 & 0.08 & 0.01 & 0.01\end{array}$

$\begin{array}{llllllllll}53.61 & 0.14 & 2.85 & 5.17 & 0.12 & 20.25 & 17.64 & 0.20 & 0.02 & 0.00\end{array}$

$\begin{array}{lllllllllll}46.09 & 0.05 & 1.68 & 9.64 & 0.17 & 36.60 & 5.68 & 0.08 & 0.02 & 0.00\end{array}$

$\begin{array}{llllllllll}45.60 & 0.06 & 1.94 & 10.29 & 0.18 & 36.82 & 5.00 & 0.11 & 0.02 & 0.00\end{array}$

$\begin{array}{llllllllll}52.20 & 1.25 & 15.56 & 10.03 & 0.22 & 6.57 & 10.21 & 3.75 & 0.10 & 0.11\end{array}$

$\begin{array}{lllllllllll}54.27 & 0.56 & 13.30 & 9.74 & 0.18 & 9.98 & 10.57 & 1.32 & 0.06 & 0.02\end{array}$

$\begin{array}{llllllllll}52.65 & 0.18 & 10.97 & 8.68 & 0.23 & 14.26 & 11.66 & 1.24 & 0.12 & 0.01\end{array}$

$\begin{array}{lllllllllll}56.36 & 0.32 & 15.19 & 8.67 & 0.15 & 7.53 & 10.20 & 1.40 & 0.15 & 0.02\end{array}$

$\begin{array}{lllllllllll}53.76 & 0.25 & 13.89 & 7.47 & 0.17 & 10.90 & 11.99 & 1.45 & 0.10 & 0.01\end{array}$

$\begin{array}{rrrrrrrrrr}54.74 & 0.24 & 11.78 & 7.78 & 0.16 & 12.42 & 12.23 & 0.61 & 0.04 & 0.01\end{array}$

$\begin{array}{lllllllllll}52.74 & 1.34 & 15.04 & 10.93 & 0.11 & 6.36 & 9.15 & 4.09 & 0.12 & 0.12\end{array}$

$\begin{array}{llllllllll}52.08 & 1.84 & 14.87 & 13.24 & 0.22 & 6.30 & 6.34 & 4.83 & 0.09 & 0.19\end{array}$

$\begin{array}{llllllllll}53.16 & 0.27 & 14.58 & 9.33 & 0.22 & 9.14 & 10.46 & 2.69 & 0.15 & 0.01\end{array}$

$\begin{array}{rrrrrrrrrr}54.99 & 0.25 & 13.46 & 8.14 & 0.17 & 10.07 & 11.58 & 1.25 & 0.08 & 0.01\end{array}$

$\begin{array}{llllllllll}57.11 & 0.41 & 15.89 & 9.99 & 0.19 & 5.01 & 9.22 & 2.07 & 0.08 & 0.02\end{array}$

$\begin{array}{llllllllll}49.08 & 0.19 & 17.31 & 5.05 & 0.10 & 11.27 & 15.89 & 1.08 & 0.02 & 0.01\end{array}$

$\begin{array}{lllllllllll}56.15 & 0.32 & 16.10 & 9.07 & 0.19 & 6.08 & 10.63 & 1.39 & 0.06 & 0.01\end{array}$

$\begin{array}{llllllllll}56.21 & 0.32 & 14.23 & 8.73 & 0.15 & 8.26 & 9.05 & 2.89 & 0.15 & 0.02\end{array}$

$\begin{array}{llllllllll}61.23 & 0.53 & 14.35 & 9.09 & 0.12 & 3.52 & 9.05 & 2.05 & 0.03 & 0.03\end{array}$

$\begin{array}{llllllllll}57.56 & 0.49 & 15.91 & 9.07 & 0.19 & 6.79 & 4.70 & 4.98 & 0.28 & 0.03\end{array}$

$\begin{array}{llllllllll}65.42 & 0.54 & 12.50 & 9.45 & 0.15 & 4.52 & 3.21 & 4.11 & 0.06 & 0.03\end{array}$

$\begin{array}{llllllllll}60.81 & 0.64 & 14.44 & 10.67 & 0.17 & 4.86 & 4.89 & 3.33 & 0.16 & 0.03\end{array}$

$\begin{array}{llllllllll}58.55 & 0.45 & 15.03 & 9.66 & 0.16 & 5.15 & 4.90 & 4.14 & 1.94 & 0.02\end{array}$

$\begin{array}{lllllllllll}59.14 & 0.46 & 14.68 & 9.87 & 0.14 & 4.28 & 6.79 & 4.05 & 0.58 & 0.02\end{array}$

$\begin{array}{llllllllll}58.32 & 0.45 & 14.63 & 10.09 & 0.15 & 4.17 & 7.55 & 4.02 & 0.59 & 0.02\end{array}$

$\begin{array}{rrrrrrrrrr}58.23 & 0.44 & 14.16 & 9.75 & 0.17 & 6.12 & 9.08 & 1.46 & 0.57 & 0.02\end{array}$

$\begin{array}{llllllllll}56.67 & 0.31 & 12.29 & 8.51 & 0.16 & 9.95 & 10.32 & 1.26 & 0.51 & 0.02\end{array}$

$\begin{array}{llllllllll}56.88 & 0.44 & 14.56 & 10.02 & 0.15 & 5.37 & 8.19 & 2.00 & 2.38 & 0.02\end{array}$

$\begin{array}{llllllllll}56.72 & 0.25 & 10.83 & 8.67 & 0.25 & 12.22 & 8.63 & 2.30 & 0.11 & 0.02\end{array}$

$\begin{array}{rrrrrrrrrr}65.16 & 1.12 & 14.41 & 7.63 & 0.11 & 2.20 & 3.01 & 6.12 & 0.03 & 0.21\end{array}$ 
Latitude Longitude

Original [wt\%]

Anhydrous [wt\%]

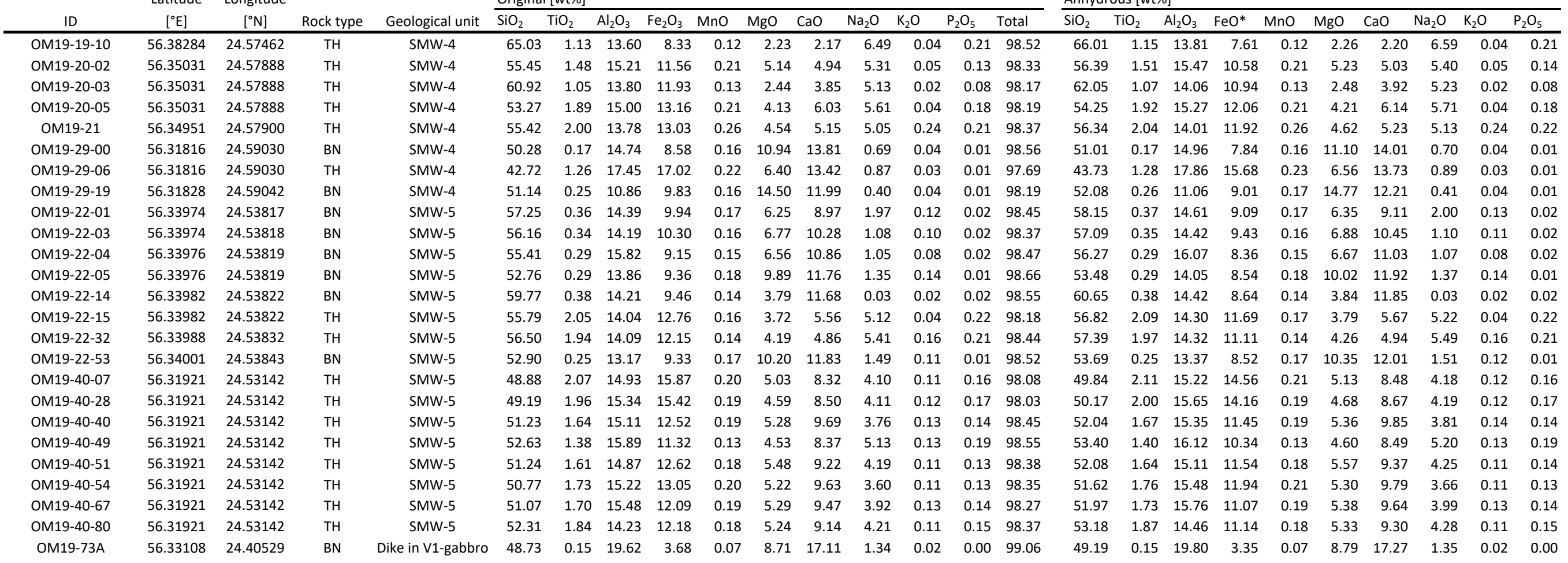




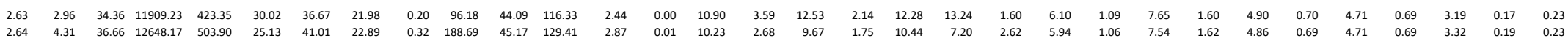

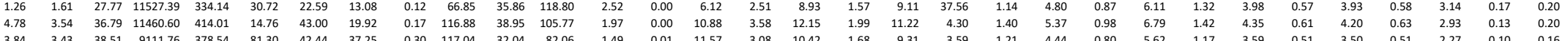

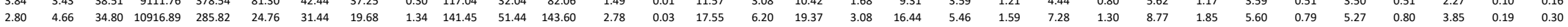

\begin{tabular}{|c|c|c|c|c|c|c|c|c|c|c|c|c|c|c|c|c|c|c|c|c|c|c|c|c|c|c|c|c|c|c|c|}
\hline & 5. & & & & & & 30.92 & 02 & & & & & 2 & & & & 2.11 & & & & & . & & 40 & & & & & 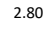 & & 0.20 \\
\hline & & & & & & & & & & & & & & & & & & & & & & & & & & & & & & & \\
\hline 2.16 & 8.51 & 36.51 & 1264.71 & 210.73 & 489.27 & 36.62 & 78.31 & 0.08 & 42.01 & 5.65 & 8.80 & 0.36 & 0.00 & 88 & 0.30 & 0.93 & 0.15 & 0 & 79 & 0. & 59 & 0.12 & 0.91 & 0.21 & 5 & b.10 & 0.70 & .11 & 0.32 & .02 & 0.05 \\
\hline 0.56 & 3.73 & 42.14 & 8087.87 & 317.30 & 130.10 & 41.42 & & 0.53 & 159.07 & 29.13 & 81.31 & 1.52 & 0.01 & 23.50 & 2.87 & 9.52 & 1.59 & 8.71 & 9.89 & 1.13 & 4.06 & 0.73 & .09 & 1.09 & 3.21 & 0.46 & 3.07 & 0.46 & 2.22 & .11 & 0.16 \\
\hline $\begin{array}{l}1.73 \\
0.69\end{array}$ & $\begin{array}{l}3.21 \\
3.11\end{array}$ & $\begin{array}{l}49.42 \\
3803\end{array}$ & $\begin{array}{l}3717.18 \\
10218\end{array}$ & $\begin{array}{l}413.43 \\
2167\end{array}$ & $\begin{array}{r}590.06 \\
\end{array}$ & 51.70 & $\begin{array}{l}154.34 \\
\end{array}$ & $\begin{array}{l}0.32 \\
0.01\end{array}$ & 65.07 & $\begin{array}{l}10.32 \\
5.1\end{array}$ & 16.33 & 0.51 & 0.01 & $\begin{array}{l}11.67 \\
\end{array}$ & 0.54 & 1.58 & 0.26 & 1.53 & 13.82 & 0.29 & 1.10 & 0.22 & 1.68 & 0.38 & $\begin{array}{l}1.25 \\
\end{array}$ & 0.19 & 1.35 & 0.21 & 0.56 & 0.04 & 0.09 \\
\hline 0. & $\begin{array}{l}3.11 \\
1.54\end{array}$ & $\begin{array}{l}38.03 \\
42.41\end{array}$ & $\begin{array}{l}10201.17 \\
1758.21\end{array}$ & $\begin{array}{l}216.75 \\
258.01\end{array}$ & $\begin{array}{r}1303 . \\
234 .\end{array}$ & $\begin{array}{l}\begin{array}{l}49.06 \\
41.70\end{array} \\
4\end{array}$ & $\begin{array}{r}225 \\
68\end{array}$ & $\begin{array}{l}0.81 \\
0.66\end{array}$ & $\begin{array}{r}51 \\
327 \\
327\end{array}$ & $\begin{array}{l}5.10 \\
8.62\end{array}$ & $\begin{array}{r}5 . \\
10 .\end{array}$ & 0 & $\begin{array}{l}0.19 \\
0.06\end{array}$ & $\begin{array}{r}4.85 \\
4588\end{array}$ & $\begin{array}{l}0.41 \\
0.46\end{array}$ & & $\begin{array}{l}.0 .15 \\
0.18\end{array}$ & $\begin{array}{l}0.76 \\
1.07\end{array}$ & $\begin{array}{l}6.90 \\
7.67\end{array}$ & $\begin{array}{l}0.17 \\
0.20\end{array}$ & & $\begin{array}{l}0 . \\
0 .\end{array}$ & 1 & $\begin{array}{l}0.18 \\
0.32\end{array}$ & $\begin{array}{l}0.61 \\
1.04\end{array}$ & 0.16 & $\begin{array}{l}0.66 \\
1.15\end{array}$ & $\begin{array}{l}0.11 \\
0.18\end{array}$ & $\begin{array}{l}0.19 \\
0.41\end{array}$ & 0.05 & $\begin{array}{l}0.06 \\
0.12\end{array}$ \\
\hline $\begin{array}{l}1.86 \\
0.33\end{array}$ & $\begin{array}{l}1.54 \\
0.41\end{array}$ & $\begin{array}{l}42.41 \\
43.88\end{array}$ & $\begin{array}{l}1 / 58.21 \\
1356.32\end{array}$ & $\begin{array}{l}248.01 \\
246.22\end{array}$ & $\begin{array}{r}2344.49 \\
1214.46\end{array}$ & $\begin{array}{l}41.10 \\
46.79\end{array}$ & $\begin{array}{r}6.8213 \\
152.98\end{array}$ & $\begin{array}{l}0.66 \\
0.63\end{array}$ & $\begin{array}{r}321.62 \\
72.91\end{array}$ & $\begin{array}{l}.862 \\
7.24\end{array}$ & $\begin{array}{r}10.96 \\
7.98\end{array}$ & 0 & $\begin{array}{l}0.06 \\
0.05\end{array}$ & $\begin{array}{l}45.88 \\
11.27\end{array}$ & $\begin{array}{l}0.46 \\
0.40\end{array}$ & $\begin{array}{l}1.18 \\
1.05\end{array}$ & $\begin{array}{l}.18 \\
0.16\end{array}$ & $\begin{array}{l}1.07 \\
0.90\end{array}$ & $\begin{array}{l}1.67 \\
19.45\end{array}$ & $\begin{array}{l}0.20 \\
0.18\end{array}$ & .73 & $\begin{array}{l}.18 \\
0.15\end{array}$ & $\begin{array}{l}1.37 \\
1.14\end{array}$ & $\begin{array}{l}.032 \\
0.26\end{array}$ & $\begin{array}{l}1.04 \\
0.86\end{array}$ & $\begin{array}{l}.016 \\
0.13\end{array}$ & $\begin{array}{l}1.115 \\
0.95\end{array}$ & $\begin{array}{l}0.18 \\
0.15\end{array}$ & $\begin{array}{l}.0 .41 \\
0.29\end{array}$ & .03 & $\begin{array}{l}0.12 \\
0.08\end{array}$ \\
\hline 2.48 & 0.34 & 38.96 & 1317.49 & 243.99 & 926.16 & & 196.35 & 0.16 & 57.37 & 6.49 & 7.04 & 43 & 0.01 & 9.40 & 0.37 & 0.95 & 0.13 & 0.74 & 9.50 & 0.23 & 52 & 0.13 & 1. & 0.24 & 0.78 & 0.12 & 87 & 0.14 & 26 & .04 & 0.09 \\
\hline 1.27 & 0.73 & 32.62 & 7362.95 & 288.71 & 90.43 & 28.14 & 39.37 & 0.62 & 153.04 & 26.10 & 71.85 & 56 & 0.02 & 14.73 & & 10.65 & 1.65 & 8.66 & 6.84 & 1.13 & 3.77 & 0.67 & 4.61 & 1.96 & 2.94 & 0.41 & 78 & 0.42 & 94 & . & $.17 \mathrm{C}$ \\
\hline & & & 9397.05 & 335.83 & 47.90 & 21.99 & Or & 0.31 & 92.57 & 41.55 & 119.82 & & .01 & 19.36 & 4.33 & 4.36 & 2.34 & 12.92 & 4.49 & 1.44 & 5.97 & 1.05 & 7.8 & & 4.58 & & 4.34 & 0.65 & 3.20 & 0.17 & 27 \\
\hline & 4.83 & 42.42 & 1505.83 & 247.23 & 538.81 & 46.99 & 100.05 & 1.01 & 110.86 & 7.42 & 8.52 & & .06 & $11.5 \mathrm{z}$ & 0.54 & 1.56 & 0.23 & 1.21 & 0.47 & 0.25 & & 0.15 & & & 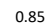 & & 0.92 & 0.15 & 0.31 & .04 & 09 \\
\hline 1.32 & 3.18 & 3 & 1358.68 & 259.01 & 652.54 & 45.47 & 116.12 & 64 & 64.95 & 7.28 & 8.44 & & 02 & 28.74 & 0.42 & 1.08 & 0.15 & 0.89 & 7.11 & 0.17 & & 0.14 & 1.1 & & 0.8 & & 94 & & & 04 & .10 \\
\hline - & 3.61 & 46.65 & 2705.25 & 378.31 & 11.07 & 40.40 & 24.35 & 0.60 & 91.34 & 11.17 & 14.59 & 0.81 & 0.04 & 59.60 & 0.60 & 1.59 & 0.24 & 1.40 & 1.96 & 0.26 & 1.14 & 0.23 & 1.74 & 0.39 & 1.30 & 0.20 & 1.44 & 0.23 & 0.52 & 0.07 & 0.14 \\
\hline 1.87 & 2. & & & 36 & & 36 & & 0.47 & & 9 & & & 0.03 & 28 & & & 1.18 & 1. & & & & 0.20 & & & & & & & & .05 & \\
\hline 1.51 & 4.31 & 45.72 & & & 309.08 & 73 & $\begin{array}{l}74.13 \\
7.678\end{array}$ & 1.44 & $\begin{array}{l}125.49 \\
2027\end{array}$ & 70 & 10.25 & 0.59 & & 34.86 & c & 1 & 0.18 & 1. & 0.61 & 0. & & 0.19 & 56 & 37 & 1.18 & & 27 & 0. & 39 & & .14 \\
\hline & 82 & $\begin{array}{l}43.12 \\
4.68\end{array}$ & $\begin{array}{l}3258.00 \\
251789\end{array}$ & $\begin{array}{l}4575.87 \\
297.79\end{array}$ & 1 & $\begin{array}{l}26.61 \\
38.13\end{array}$ & & $\begin{array}{l}0.24 \\
3.22\end{array}$ & $\begin{array}{l}203 . \\
110 .\end{array}$ & $\begin{array}{l}15.51 \\
1301\end{array}$ & $\begin{array}{l}22.41 \\
17.30\end{array}$ & 0 & $\begin{array}{l}0.01 \\
0.19\end{array}$ & $\begin{array}{r}9.04 \\
2888\end{array}$ & $\begin{array}{l}0.91 \\
0.75\end{array}$ & 2 & $\begin{array}{l}0.38 \\
0.32\end{array}$ & 2. & & 0. & & 0 & & 0.54 & 1.77 & & $\begin{array}{l}1.95 \\
1.68\end{array}$ & 32 & 72 & 108 & .20 \\
\hline & $\begin{array}{l}5.82 \\
4.76\end{array}$ & $\begin{array}{l}42.28 \\
12.21\end{array}$ & $\begin{array}{l}21977.89 \\
2417.00\end{array}$ & $\begin{array}{l}297.79 \\
329.33\end{array}$ & $\begin{array}{l}29.16 \\
24.47\end{array}$ & $\begin{array}{l}38.13 \\
38.93\end{array}$ & $\begin{array}{l}36 \\
21\end{array}$ & $\begin{array}{l}3.22 \\
0.37\end{array}$ & $\begin{array}{r}1110.50 \\
17.08\end{array}$ & $\begin{array}{r}13.01 \\
2.21\end{array}$ & $\begin{array}{r}17.30 \\
6.00\end{array}$ & & $\begin{array}{l}0.19 \\
0.03\end{array}$ & $\begin{array}{r}28.88 \\
5.46\end{array}$ & $\begin{array}{l}0.75 \\
0.39\end{array}$ & 1.74 & $\begin{array}{l}0.322 \\
0.20\end{array}$ & $\begin{array}{l}1.82 \\
0.91\end{array}$ & & $\begin{array}{l}0.30 \\
0.10\end{array}$ & & .07 & & & 1.54 & & & & & & .06 \\
\hline 5.24 & 4.48 & 35.66 & 3378.45 & 427.09 & 8. & 34.87 & & 05 & 81.19 & $\begin{array}{l}2.11 \\
13.11\end{array}$ & 21.68 & & 14 & $\begin{array}{r}25.29 \\
25.29\end{array}$ & & & 0.36 & 2. & & & & & & & & & & & & & \\
\hline 5.81 & 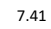 & & & & 33.80 & 32.70 & & 26.34 & 109.37 & 11.44 & 14.46 & & & & & & & & & & & & & & & & & & & & \\
\hline 4.65 & 9.31 & 48.89 & 306 & 487.61 & 79. & 34.86 & 25 & 24 & 67.43 & 12.98 & 16.49 & & 3 & & & & 0. & 1 & & & & & & & & & & & & & 46 \\
\hline & 7.80 & & & & 62 & 33 & & 11 & 79 & 12 & 14.78 & & & & & & & & & & & & & & & & & & & & \\
\hline 5.38 & 4.76 & 45.33 & & & 11 & 40.11 & 13 & 10 & 72 & 9 & 11. & & & 102.68 & & & & & & & & & & & & & & & & & \\
\hline 4. & 4. & 51.90 & 187 & 312.30 & 63 & 45.32 & 124.42 & 7.55 & 86. & 7.24 & 8.88 & & 0.11 & 51.46 & & 2. & & 1. & & & & & & & & & & 0.16 & & & \\
\hline & 10.04 & & & 469.70 & 12 & 42.09 & 49.40 & 40.04 & 250 & 11. & 14. & & & 251.68 & & & & & & & & & & & & & & & & & \\
\hline 4.c & 4.83 & 52.16 & 145 & 277.65 & 854.82 & 73 & 186.24 & 1.12 & 84. & 7.30 & 10 & & 0.6 & 11.06 & & & & & & & & & & & & & & & 16 & & \\
\hline 0.7 & 5.24 & 17.63 & & 52 & 6. & 64 & 4. & 0 & 102. & 54.65 & 18 & & & 4. & & & & & & & & & & & & & & & & & \\
\hline 0.7 & 3.22 & 15.12 & & (3. & 5. & 13 & 4. & 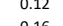 & & 47 & 16 & & & $6.9 \mathrm{I}$ & & & & & & & & & & & & & & & & & 50 \\
\hline 0.98 & 5.06 & 26 & 52 & 362 & 12. & 19 & ${ }_{9}^{13}$ & 0 & 135 & $\begin{array}{l}3.2 .69 \\
25.94\end{array}$ & & & & 7.01 & & ${ }_{6}^{11}$ & & 1 & & & & & & & & & & & & & \\
\hline 0.4 & 4.60 & 2 & & & 8 & 1575 & 12.24 & 0.09 & 9494 & 47 & 113.17 & & 0 & 5. & 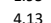 & 13. & 222 & 11. & & & & & & & & & & & 01 & & \\
\hline 0.8 & 2.72 & 27.18 & 3.87 & .04 & 29.77 & 20.84 & 0.60 & 1.90 & 136.64 & 49.31 & 170.88 & 3.37 & 0.05 & 17.30 & .79 & 15.68 & 60 & 1 & 6.50 & 1.55 & 6.69 & 1.19 & 8.29 & 1.74 & 5.40 & 0.77 & 5.13 & 0.79 & 4.61 & .24 & 37 \\
\hline
\end{tabular}

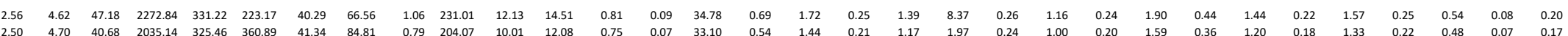

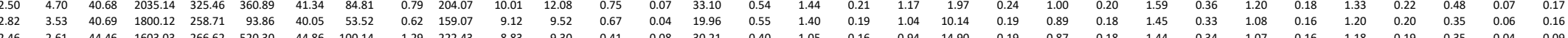

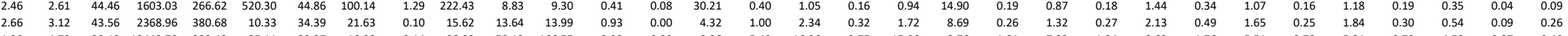
$\begin{array}{rrrrrrrrrrrrrrrrrrrrrrrrrrrrrrrr}1.30 & 4.72 & 29.19 & 13449.70 & 333.43 & 25.11 & 22.27 & 16.98 & 0.14 & 96.09 & 50.12 & 166.55 & 3.93 & 0.00 & 8.96 & 5.43 & 16.96 & 2.75 & 15.06 & 8.76 & 1.81 & 7.02 & 1.24 & 8.63 & 1.76 & 5.51 & 0.79 & 5.31 & 0.79 & 4.50 & 0.27 & 0.40 \\ 1.00 & 5.26 & 26.73 & 11570.01 & 310.13 & 11.46 & 26.58 & 13.84 & 1.18 & 122.68 & 44.99 & 142.58 & 3.53 & 0.04 & 14.43 & 4.93 & 16.15 & 2.57 & 13.89 & 20.82 & 1.75 & 6.33 & 1.13 & 7.67 & 1.58 & 4.95 & 0.70 & 4.79 & 0.71 & 3.79 & 0.24 & 0.37\end{array}$

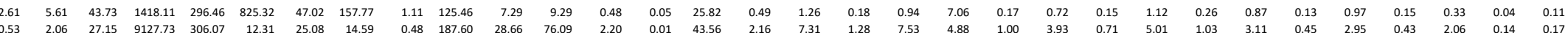

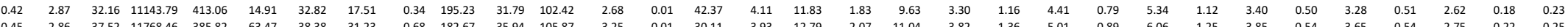

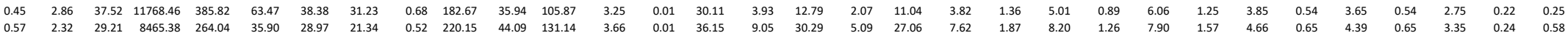

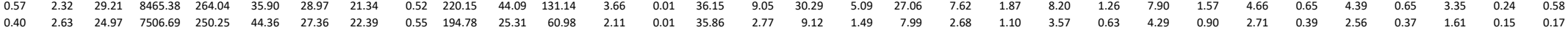
$\begin{array}{llllllllllllllllllllllllllllllll}0.41 & 1.84 & 35.44 & 13010.61 & 410.78 & 66.79 & 41.32 & 32.70 & 0.38 & 178.37 & 35.36 & 145.28 & 4.10 & 0.01 & 32.26 & 3.93 & 13.27 & 2.09 & 11.20 & 3.94 & 1.36 & 4.96 & 0.89 & 6.10 & 1.27 & 3.86 & 0.55 & 3.81 & 0.57 & 3.29 & 0.28 & 0.33 \\ 0.62 & 2.55 & 29.03 & 9153.76 & 300.34 & 53.29 & 31.08 & 27.50 & 0.65 & 200.17 & 30.00 & 80.47 & 2.48 & 0.01 & 29.26 & 4.93 & 13.61 & 2.06 & 10.66 & 3.41 & 1.25 & 4.38 & 0.77 & 5.30 & 1.10 & 3.25 & 0.45 & 3.09 & 0.45 & 2.05 & 0.16 & 0.26 \\ 0\end{array}$

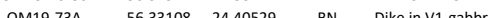

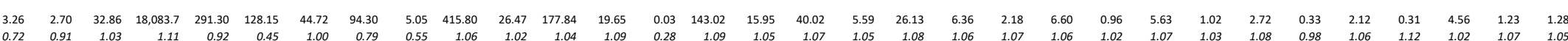

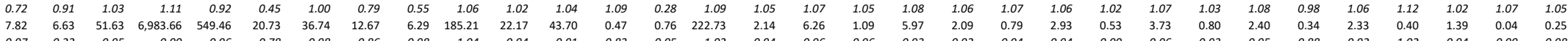

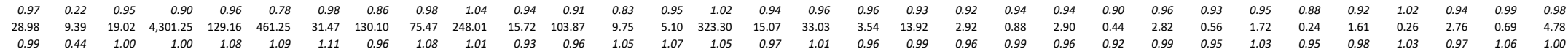

